\title{
CUBESAT MISSION PLANNING TOOLBOX
}

\author{
A Thesis \\ presented to \\ the Faculty of California Polytechnic State University, \\ San Luis Obispo
}

\author{
In Partial Fulfillment \\ of the Requirements for the Degree \\ Master of Science in Aerospace Engineering \\ by \\ Brian Rand Castello \\ June 2012
}


(C) 2012

Brian Rand Castello

All Rights Reserved 


\section{COMMITTEE MEMBERSHIP}

TITLE: CubeSat Mission Planning Toolbox

AUTHOR: Brian Castello

DATE SUBMITTED: June 7, 2012

COMMITTEE CHAIR: Dr. Kira Abercomby, Assistant Professor

COMmitTeE MEMBER: Dr. Eric Mehiel, Associate Professor

COMMitTEE MEMBER: Dr. John Bellardo, Associate Professor

COMMITTEE MEMBER: Daniel Wait, Lecturer 


\section{Abstract \\ CubeSat Mission Planning Toolbox \\ Brian Rand Castello}

We are in an era of massive spending cuts in educational institutions, aerospace companies and governmental entities. Educational institutions are pursuing more training for less money, aerospace companies are reducing the cost of gaining flight heritage and the government is cutting budgets and their response times. Organizations are accomplishing this improved efficiency by moving away from large-scale satellite projects and developing Pico- and Nano-satellites following the CubeSat Specifications. One of the major challenges of developing satellites to the standard CubeSat mission requirements is meeting the exceedingly tight power, data and communication constraints.

A MATLAB toolbox was created to assist the CubeSat community with understanding these restrictions, optimizing their systems, increasing mission success and decreasing the time building to these initial requirements. The Toolbox incorporated the lessons learned from the past nine years of CubeSats' successes and Analytical Graphics, Inc. (AGI)'s Satellite Tool Kit (STK). The CubeSat Mission Planning Toolbox (CMPT) provides graphical representations of the important requirements a systems engineer needs to plan their mission. This includes requirements for data storage, ground station facilities, orbital parameters, and power. CMPT also allows for a comparison of broadcast (BC) downlinking to Ground Station Initiated (GSI) downlinking for payload data using federated ground station networks. Ultimately, this tool saves time and money for the CubeSat systems engineer. 


\section{Acknowledgements}

This thesis would not have been possible without the support of my friends and family. I would also like to thank the PolySat teams past and present. Their knowledge and guidance have propelled my work forward and nothing in this paper would have come together without them. 


\section{Contents}

List of Figures $\quad$ xi

List of Tables $\quad$ XV

Glossary xvii

1 Scope of Thesis 1

1.1 Audience . . . . . . . . . . . . . . . . . . 2

2 Introduction $\quad 3$

2.1 Background .......................... 3

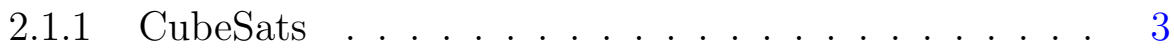

2.1.2 Trends . . . . . . . . . . . . . . . . 4

2.1.3 Systems Engineering Trends . . . . . . . . . . . 4

2.1.4 Educational Launch of Nanosatellites (ELaNa) . . . 5

2.2 CubeSat Communications . . . . . . . . . . . . 6

2.2.1 Amateur Frequencies . . . . . . . . . . . . . 6

2.2.2 Amateur Radio Ground Stations . . . . . . . . 7

2.2 .3 AX.25 . . . . . . . . . . . . . 9

2.2.4 Global Educational Network for Satellite Operations (GENSO) . . . . . . . . . . . . . . . . 9 9

2.2.5 Federated Ground Station Networks . . . . . . . . . . 10

2.2.6 Downlinking Methods . . . . . . . . . . 11

$2.3 \mathrm{STK} \ldots \ldots \ldots \ldots \ldots \ldots \ldots$

2.3.1 Interfacing . . . . . . . . . . . . . . . 12

2.3 .2 Scenarios . . . . . . . . . . . . . 13

2.3 .3 Objects ...................... 13

2.3 .4 Access Reports . . . . . . . . . . . . 15 
2.3.5 Interoperability and Integration Module (IIM) . . . 15

2.3.6 Connect Commands . . . . . . . . . . . . 16

3 CubeSat Survey 19

3.1 Research . . . . . . . . . . . . . . . . . . . 19

3.2 Mission Success and Failure . . . . . . . . . . . . 21

3.3 Defining Characteristics of Past Missions . . . . . . . . . . 21

3.3.1 Planned Mission Length . . . . . . . . . . . . . . 21

3.3 .2 Size . . . . . . . . . . . . . . . 22

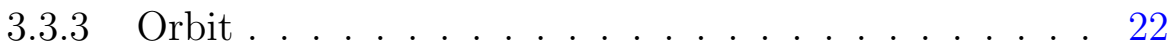

3.3.4 Mission Description . . . . . . . . . . . . . . . 23

3.3.5 Data Rate . . . . . . . . . . . . . . 23

3.3.6 Transmitter Power . . . . . . . . . . . . . . . 24

3.4 Categories . . . . . . . . . . . . . . . . 25

3.4 .1 Initial Attempts . . . . . . . . . . . . . . . 25

3.4 .2 One-Shot . . . . . . . . . . . . . 25

3.4.3 Science Missions . . . . . . . . . . . . . . 27

3.4 .4 Test-Beds . . . . . . . . . . . . . . . . 28

3.4.5 Custom . . . . . . . . . . . . . . . . . . 28

3.4.6 Matrix of Mission Parameters . . . . . . . . . . . . 29

4 CubeSat Mission Planning Toolbox 31

4.1 CMPT Setup . . . . . . . . . . . . . . . . 31

4.1 .1 Mission Type . . . . . . . . . . . . . . 31

4.1 .2 Time of Test . . . . . . . . . . . . . . . . . 33

4.1.3 Ground Stations Options . . . . . . . . . . . 34

4.1.4 Orbital Options . . . . . . . . . . . 36

4.1 .5 Satellite Information . . . . . . . . . . . . 38

4.1 .6 Data Specific . . . . . . . . . . . . . . . . 41

4.1 .7 Transmitter Information . . . . . . . . . . . . 43

4.2 CMPT Summary . . . . . . . . . . . . . . . . . 49

4.2.1 Summary of Options Panel . . . . . . . . . . . 49

4.2 .2 Times Panel . . . . . . . . . . . . . . . . . . . . 51

4.2 .3 Initial Ground Station Results . . . . . . . . . . . . 52

4.2.4 Graphs Panel . . . . . . . . . . . . . . . . . 54 
4.2.5 Specifics Panel . . . . . . . . . . . . . 55

4.2.6 Status and Warnings . . . . . . . . . . . . 56

4.3 CMPT Printing and Suggestions . . . . . . . . . . . . 57

4.3.1 Suggested Results . . . . . . . . . . . . . . 58

4.3.2 Print Options and Sample Output. . . . . . . . . 59

4.3 .3 Graphs................... 60

4.4 Calculations . . . . . . . . . . . . . . . . 61

4.4.1 Time Calculations Introduction . . . . . . . . . 61

4.4.2 Downlink Time . . . . . . . . . . . . . . . . 61

4.4 .3 Total Access Time . . . . . . . . . . . . . . . . 61

4.4.4 longest Access Period . . . . . . . . . . . . 61

4.4.5 Electrical Calculations Introduction . . . . . . . . . 62

4.4.6 Power Required for Downlinking . . . . . . . . . . . . 62

4.4 .7 Current Density . . . . . . . . . . . . . . . 65

4.4.8 Percent of Battery Used for Transmission . . . . . . . 65

4.4.9 Data Calculations Introduction . . . . . . . . 66

4.4.10 Total Packet Bundle Size . . . . . . . . . . . . . . . 66

4.4.11 Number of Packets . . . . . . . . . . . . . . . 66

4.4.12 Percentage of Data Downlinked . . . . . . . . . 67

4.4 .13 Suggested Data Storage Size . . . . . . . . . . 67

5 Case Studies $\quad 69$

5.1 Base Cases . . . . . . . . . . . . . . . . . 70

5.1 .1 Initial Attempt . . . . . . . . . . . 70

5.1 .2 One-Shot . . . . . . . . . . . . 71

5.1 .3 Science Mission . . . . . . . . . . . . . . 72

5.1 .4 Industry Test Bed . . . . . . . . . . . . . . 73

5.1.5 Summary of Suggestions . . . . . . . . . . . 74

5.2 Real World Cases . . . . . . . . . . . . . . . . . . . 75

5.2 .1 Initial Attempt: CP6 . . . . . . . . . . 76

5.2 .2 One Shot: CP5 . . . . . . . . . . . . . 77

5.2 .3 Science: QuakeSat . . . . . . . . . 78

5.2 .4 Science: RAX-2 . . . . . . . . . . . . . . 79

5.3 Results . . . . . . . . . . . . . . . 80

$5.3 .1 \mathrm{CP} 6 \ldots \ldots \ldots \ldots \ldots$ 
$5.3 .2 \quad \mathrm{CP} 5 \ldots \ldots \ldots \ldots \ldots$

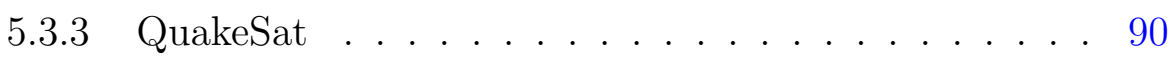

5.3 .4 RAX-2 . . . . . . . . . . . . . . 94

6 Conclusion $\quad 99$

6.1 Validation . . . . . . . . . . . . . . . . . . . 99

6.1.1 Link Budget Calculation . . . . . . . . . . . . . . . 99

6.1.2 Single Station Mission . . . . . . . . . . . . . 101

6.1.3 Full World Mission . . . . . . . . . . . . . . . . 101

6.2 Success of CMPT . . . . . . . . . . . . . . . . . . 102

6.3 Success Against CubeSat Case Studies . . . . . . . . . . . . 102

6.4 Future Work . . . . . . . . . . . . . . . . . . . . . 103

$\begin{array}{ll}\text { References } & 105\end{array}$

A Procedures for Acquiring Amateur Frequency License 111

A.1 IARU Coordination Request Form . . . . . . . . . . . . . . . 113

A.2 FCC Summary Template . . . . . . . . . . . . . . . . . . . 123

B Cubesat Survey Matrix $\quad 129$

$\begin{array}{ll}\text { C CMPT User Guide } & 137\end{array}$

D Useful MATLAB Code 143

$\begin{array}{ll}\text { E CP6 Real World Data } & 149\end{array}$ 


\section{List of Figures}

2.1 Coverage area of the RAX-2 ground station network. . . . 8

2.2 North America Scenario of STK . . . . . . . . . . . . . 13

2.3 Object options of STK . . . . . . . . . . . . . . . . . . 14

2.4 Access Report in STK . . . . . . . . . . . . . . . . 15

2.5 STK Initialization MATLAB code . . . . . . . . . . . . . 16

2.6 MATLAB code to load scenario . . . . . . . . . . . . . 16

2.7 MATLAB code to add object . . . . . . . . . . . 17

4.1 Screenshot of CMPT Setup Window . . . . . . . . . . . . 32

4.2 Close up of the Mission Type Panel . . . . . . . . . . . . . . 32

4.3 Close up of the Time of Test Panel . . . . . . . . . . . . . . 33

4.4 Close up of the Ground Station Panel . . . . . . . . . . . . . 34

4.5 Coverage area of the North American Ground Station network 35

4.6 Coverage area of the RAX-2 ground station network. . . . . 36

4.7 Close up of the Orbits Option Panel . . . . . . . . . . . . . 36

4.8 Close up of the Satellite Info panel . . . . . . . . . . . . . 38

4.9 Close up of the Data Specifics Panel . . . . . . . . . . . . . 41

4.10 Close up of the Transmitter Info Panel . . . . . . . . . . . . 43

4.11 BER vs. $E_{b} / N_{0} \ldots \ldots \ldots \ldots$

4.12 Screenshot of the CMPT Summary Window . . . . . . . . . 49

4.13 Screenshot of the Summary of Options Panel . . . . . . . . . 49

4.14 Screenshot of the Times Panel . . . . . . . . . . . . . . . 51

4.15 Screenshot of the Initial Ground Station Results Panel . . . 52

4.16 Screenshot of the Graphs Panel . . . . . . . . . . . . . . . 54

4.17 Screenshot of the Specifics Panel . . . . . . . . . . . . 55

4.18 Screenshot of the Status and Warnings Panels . . . . . . . 56

4.19 Screenshot of CMPT Print Window . . . . . . . . . . . . 57 
4.20 Screenshot of the Suggestion section in the Print Menu Win-

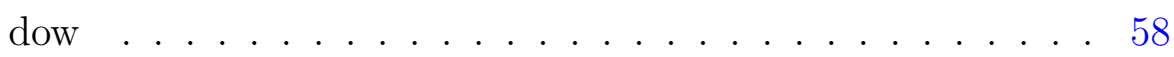

4.21 Output of CMPT print in tabular Form. . . . . . . . . . . 59

4.22 Screenshot of the Access vs. SMA vs. Inc in minutes . . . . 60

4.23 Visualization of Slant with an Airplane . . . . . . . . . . 63

5.1 Screenshot of print menu for the Initial Attempt mission type 70

5.2 Screenshot of Print Menu for the One-Shot mission type . . 71

5.3 Screenshot of Print Menu for the Science mission type . . . . 72

5.4 Screenshot of Print Menu for the Industrial Test-Bed Mission

Type ......................... 73

5.5 Image of $\mathrm{CP} 6 \ldots \ldots \ldots 76$

5.6 Screenshot of CP6 CMPT setup window . . . . . . . . 76

5.7 CP5 mission badge . . . . . . . . . . . . . . . 77

5.8 Screenshot of CP5 CMPT setup window . . . . . . . . . 77

5.9 QuakeSat mission badge . . . . . . . . . . . 78

5.10 Screenshot of QuakeSat CMPT setup window . . . . . . . 78

5.11 RAX-2 . . . . . . . . . . . . . . . 79

5.12 Screenshot of RAX-2 CMPT setup window . . . . . . . . 79

5.13 Screenshot of CP6 Print Screen . . . . . . . . . . . . . 81

5.14 Screenshots of Percent Downlinked Plots for CP6 . . . . . . 82

5.15 Screenshots of the Power Density Plots for CP6 . . . . . . . 84

5.16 Screenshots of the Storage Required Plots for CP6 . . . . . . 85

5.17 Screenshot of CP5 Print Screen . . . . . . . . . . . . 86

5.18 Screenshots of Percent Downlinked Plots for CP5 . . . . . . 87

5.19 Screenshots of the Power Density Plots for CP5 . . . . . . 88

5.20 Screenshots of the Storage Size Plots for CP5 . . . . . . . . 89

5.21 Screenshot of QuakeSat Print Screen . . . . . . . . . . . . . 90

5.22 Screenshot of Shortest Access for QuakeSat . . . . . . . . . . 91

5.23 Screenshot of Percent Downlinked for QuakeSat . . . . . . . 91

5.24 Screen Shot of the Power Density Plot for GSI Downlink . . 92

5.25 Screen Shot of RAX-2 Print Screen . . . . . . . . . . . . 94

5.26 Screenshots of Percent Downlinked Plots for RAX-2 . . . . . 95

5.27 Screenshots of the Power Density Plots . . . . . . . . . . 96

5.28 Screenshots of the Storage Required Plots for RAX-2 . . . . 97 
D.1 MATLAB code used to run the simulation . . . . . . . . 144

D.2 MATLAB code used to get the Access Reports from STK. . 145

D.3 MATLAB code used to convert the Access Reports from STK.146

D.4 MATLAB code used to find the longest access period in each orbit. . . . . . . . . . . . . . . . . . 147 


\section{List of Tables}

2.1 ELaNa Program Summary . . . . . . . . . . . . . . . . 6

3.1 Parameters for Initial Attempt satellites . . . . . . . . . . . 26

3.2 Parameters for One-Shot satellites . . . . . . . . . . . . . . 27

3.3 Parameters for Science satellites . . . . . . . . . . . . . . . . 28

3.4 Parameters for Test-Bed satellites . . . . . . . . . . . . . . . 29

3.5 Matrix of the parameters for each of the satellite categories . 29

4.1 Orbital Options description . . . . . . . . . . . 38

4.2 Battery Options . . . . . . . . . . . . . . . . . . 41

4.3 Parameters and references for each of the Satellite Parameters 50

4.4 Title and axis of the Graphs Panel . . . . . . . . . . . . 55

4.5 The Specifics Panel Outputs and their Reference . . . . . . . 55

4.6 The Status and Warnings Section Summary . . . . . . . . 56

5.1 Summary of Suggests for each base case. . . . . . . . . . . . 74

5.2 Summary of Real World Cases . . . . . . . . . . . . . . . . . 75

5.3 Parameters for the CP6 mission . . . . . . . . . . . 76

5.4 Parameters for the CP5 mission . . . . . . . . . . . . . 77

5.5 Parameters for the QuakeSat mission . . . . . . . . . . 78

5.6 Parameters for the RAX-2 mission . . . . . . . . . . . . . 79

5.7 Comparsion values for GSI and BC methods of CP6 . . . . . 81

5.8 Comparsion values for the GSI and BC methods of CP5 . . 86

5.9 The Results of the QuakeSat Mission . . . . . . . . . . . . . 90

5.10 Comparsion values for GSI and BC methods of RAX-2 . . . 94

6.1 Comparsion of CP6 Data and CMPT output . . . . . . . . . 101 


\section{Glossary}

$1 \mathrm{U}$

A $1 \mathrm{U}$ CubeSat is the smallest CubeSat with dimensions of $10 \mathrm{~cm}$ by $10 \mathrm{~cm}$ by $10 \mathrm{~cm} .2,16,19,22,66,67$

$2 \mathrm{U}$

A $2 \mathrm{U}$ CubeSat is a medium size CubeSat with dimensions of $10 \mathrm{~cm}$ by $10 \mathrm{~cm}$ by $20 \mathrm{~cm} .2,16$

$3 \mathrm{U}$

A 3U CubeSat is the largest CubeSat that fits in the current P-POD with dimensions of $10 \mathrm{~cm}$ by $10 \mathrm{~cm}$ by $30 \mathrm{~cm} .2,16,22$

AGI

Analytical Graphics, Inc. is the company that makes STK. 9

\section{altitude}

The altitude is the height of the satellite in $\mathrm{km}$ above the earths surface. $17,30,31,43,53,56$

\section{APRS}

Automatic Packet Reporting System is a two-way tactical real-time digital communications system between all assets in a network sharing information about everything going on in the local area [1]. 6 
AX.25

The AX.25 packet format is widely used in the amateur radio community for data transmission [2]. 6, 56

\section{bandwidth}

The bandwidth is essentially the rate of data transfer. 21

\section{baud rate}

The baud rate is the symbols per second or pulses per seconds. The number of distinct symbol changes made transmission medium per second. Baud Rate will be measured in bits per seconds (bps) in this thesis. 6

\section{BC}

The broadcast downlinking method consists of continous transmission over the period of operation. $7,8,59,65,70-79,82-85,87$

\section{BER}

Bit error rate is the principal performance metric that is a measure of quantization error in digital communications links. 37,38

\section{BPSK}

Binary phase shift keying encodes information onto carriers through the use of two phase states per symbol, thereby encoding 1 bit fo information per symbol [3]. 28

\section{bus voltage}

The bus voltage is the main voltage the satellite runs on. There may be multiple voltages present in a system, select the one that the communication system if there are variations. $32,33,55$ 
Cal Poly

California Polytechnic State University, San Luis Obispo. 2, 3, 67

\section{CMPT}

The CubeSat Mission Planning Toolbox is a MATLAB program that uses STK to assist CubeSat Developers with system planning. 1, 9, $13,15,19,22,25-28,31,36,40,44,46-51,53,56,59-63,65-69,74$, $78,80,81,85,87,88$

\section{CubeSat}

A CubeSat is a type of miniaturized satellite for space research that usually has a volume of exactly one liter (10 cm cube), weighs no more than one kilogram, and typically uses commercial off-the-shelf electronics components. 1-7, 15, 22, 25, 26, 30-34, 36, 38, 49, 55-57, $65,67,81,88$

\section{current density}

Current density in this thesis represents the amount of current used over a particular time period. The units of current density in this thesis are amp-hours. 32

$\mathrm{dB}$

A decibel is a base ten logarithmic unit that describes the ratio of a physical quantity (such as the power of an electromagnetic wave) relative to a specificed or implied reference level [3] . 4, 19-23, 36-39, $53-55$

\section{$\mathrm{dBm}$}

A dBm is a decibel referenced to one milliwatt. 4, 19-23, 44, 46, 55, 
$60-64$

\section{decoder}

A decoder is a software package that allows a ground station operator the ability to capture and display data from the satellite. 5, 28

\section{eccentricity}

Eccentricity is a convenient ratio to parameterize all the conic sections of a possible orbit [3]. 31

\section{ELaNa}

ELaNa is a NASA initiative that provides opportunities for educational CubeSats on every unmanned National Aeronautics and Space Administration (NASA) launch. 3, 4, 30

\section{ESA}

The European Space Agency is Europes gateway to space. 7

\section{FCC}

The Federal Communications Commission regulates interstate and international communications by radio, television, wire, satellite and cable . 4, 28

\section{gain}

Antenna gain described mathematically as the power density radiated in a given direction relative to the power density that would have been radiated in that direction by an isotropic radiator [3]. 36, 37, 54

\section{GENSO}

The main goal of GENSO is to provide a worldwide network of radio 
amateur and university ground stations to support the operations of university satellites. 7,88

\section{GEO}

A geosynchronous is an orbit at $35,856 \mathrm{~km}$ and zero degrees of inclination. 17

\section{GLORY}

NASA's GLORY satellite is a low Earth orbit scientific research satellite mission which will increase our understanding of the Earth's energy balance. This mission was also NASA first attempt providing CubeSats with launch opportunities. 3, 4

\section{GSI}

Ground station initiated downlinking requires the main ground station to initiate the downlinking of data. 7, 8, 59, 65, 70-79, 82-85, 87

\section{IARU}

The International Amateur Radio Union is the international regulatory organization that allocates radio frequencies. 4

\section{IIM}

The Interoperability and Integration module provides interfacing capabilities to STK. 11

\section{inclination}

Inclination is the angle between the orbit plane and a reference plane which also contains the center of mass [3]. 31, 43, 56 
LEO

The low earth orbits are orbits between $150 \mathrm{~km}$ and 1,000 km [4]. 16, $17,23,31,60-63,65$

\section{link budget}

A link budget is used to determine the correct parameters for an effective communication link. 53, 54

\section{MATLAB}

MATLAB is a programming environment for algorithm development, data analysis, visualization, and numerical computation. 9, 11-13, $25,45,52,88$

\section{MEO}

The medium earth orbits are orbits between $3,000 \mathrm{~km}$ and $35,856 \mathrm{~km}$. $17,23,31$

\section{More dBs}

More $\mathrm{dBs}$ is a system to manage all data generated by Cal Poly satellites during development and on-orbit operations [5]. 5, 29, 66, 67

\section{NPP}

NASA's NPP satellite is a next-genreation Earth-observing satellite. This mission took five CubeSats into orbit [6]. 4

\section{ORS Enabler}

The Operationally Responsive Space Enabler mission was going to provide university CubeSats with a launch opportunity. 4 


\section{P-POD}

The P-POD is designed to provide a standard secondary payload interface between CubeSats and launch vehicles. 2-4

\section{pecuniary interest}

Pecuniary interest is having some financial interest such as profit or selling some part of the project. 5

\section{RF AMP}

The radio frequency amplifier is a component designed to increase the power of the communication signal before transmitting the signal through the antenna. 36

\section{S band}

The $\mathrm{S}$ band is the range of radio frequencies from 2 to $4 \mathrm{GHz} .36$

\section{satellite buses}

The satellite bus is the main components of a satellite such as the communication system, power system and other components. 3

\section{SMA}

The semi-major axis is one half of the major axis, and thus runs from the centre, through a focus, and to the edge of the ellipse.. 51

\section{STK}

The Satellite Tool Kit is a general-purpose modeling and analysis application for any type of space, defense or intelligence system [7]. xvi, 1, 9-12, 25, 29, 47, 88, 89 


\section{Glossary}

\section{TUI's Hoytether technology}

The Tethers Unlimited Incorporated's Hoytether is a tether structure composed of multiple lines with redundant interlinking that is able to withstand many impacts [8]. 2

yagi

A yagi antenna is a highly directional antenna typically used in amateur radio communications. 37 


\section{1}

\section{Scope of Thesis}

This thesis discusses many previous CubeSat missions from a systems engineering point of view. These missions are assessed and four categories of CubeSat missions are defined. Each CubeSat mission category establishes individual baseline requirements meant to best address those past missions. The CMPT uses these requirements to suggest a variety of parameters for different CubeSat missions. These requirements and suggestions are designed to guide the early stages of CubeSat development and provide insight into how the system will perform during operations.

Chapter 3 discusses the development of the categories of CubeSats and defines the requirements for each of the four categories.

Chapter 4 explains the user interface, the formulas used and how CMPT displays the results. This chapter also includes an explanation of how STK calculates access times and the steps to determine transmission power requirements. 
Chapter 5 analyzes the use of CMPT for the four mission types defined in chapter 3. In this chapter, fours real world missions are examined to demonstrate the application of CMPT. Finally, this chapter discusses CMPT's suggested improvements to any of the case study missions.

Chapter 6 discusses the successes of CMPT and possible improvements to CMPT.

\subsection{Audience}

This thesis is geared towards organizations that are developing low cost CubeSat satellites and have access to STK licenses such as the Integration module. Cal Poly's PolySat program is an excellent example of an organization that can use and benefit from this thesis. The acquistion of STK and separate licenses can be thousands of dollars which might not be acceptable to organizations that do not have access to educational licenses. CMPT's dependency on STK will be assessed in future versions of the program. 
2

\section{Introduction}

\subsection{Background}

\subsubsection{CubeSats}

In 1999, Jordi Puig-Suari of California Polytechnic State University, San Luis Obispo (Cal Poly) and Bob Twiggs of Stanford University University scribbled some ideas down on a napkin. These ideas led a handful of Cal Poly students to design a simple aluminum box qualified for space. This box is called the Poly-PicoSatellite Orbital Deployer (P-POD). The P-POD holds three 10x10x10 cm satellites known as CubeSats. Initially, these small sized satellites would only be able to be used as interesting school projects for aerospace students. However, after that first batch of CubeSats were integrated in 2003 to the Rokot Rocket [9] and the engines fired up, the world realized that having less expensive launch opportunities could benefit not only universities, but corporations and even government institutions as well. Since that first initial launch, numerous organizations have joined the CubeSat Community. In the last nine years, there have 


\section{INTRODUCTION}

been 10x10x10 cm (1U), 10x10x20 cm (2U)), and further 10x10x30 cm (3U)satellites launched with the P-POD.These satellites test everything from simple cell phone cameras [10] to complex studies of the ionosphere using multi-spectral cameras [11].

\subsubsection{Trends}

In the early stages of the CubeSat program, the CubeSat Satellites were developed to understand the technology. The early payloads were developed to generate enough data to test the system and validate their communication capabilities. However, as government institutions and the National Science Foundation saw the success of early CubeSats, these institutions worked to support the next generation of CubeSat with a focus on more complex science missions such as DICE which measured ionospheric plasma densities [12]. While there are many complex science missions, there are also CubeSat with a single-purpose mission such as MAST which is a satellite mission consisting of three tethered CubeSats developed to test the TUI's Hoytether technology [8]. While most of the CubeSat community consists of university and government CubeSats, there are a few industry generated CubeSats. These mission, such as CSTB-1 [13], are funded by industry leaders like Boeing.

\subsubsection{Systems Engineering Trends}

Another aspect of early CubeSats was engineering dominated by the use of inexpensive parts. These practices affected everything from battery design to link budget margins. However, as the CubeSat technologies advanced and the engineering matured, avionics has decreased in size to make room 
for larger, more powerful payloads, and more flexible satellite buses. Cal Poly's latest Avionics package [14] provides an example of these next generation trends, which consist of providing an optimized avionics system with a maxmium for payload capabilities.

\subsubsection{Educational Launch of Nanosatellites (ELaNa)}

In 2010, NASA flew a group of CubeSats on the NASA 's GLORY launch. Although this launch ultimately came to an unfortunate end when the mission failed to reach orbit, NASA was able to test the P-POD and it functioned as expected. Out of the destruction of GLORY came the success and birth of the Educational Launch of Nanosatellites (ELaNa) program. ELaNa is a NASA initiative that provides opportunities for educational CubeSats on every unmanned NASA launch [15]. One major aspect of the program is to reduce costs to the university associated with integration and testing. The reduction of cost to the university is a two-part process. The first part uses Cal Poly's CubeSat program to do a majority of the preintegration and testing work, with NASA providing oversight and approval procedures. Utilizing Cal Poly's CubeSat program further involves students and reduces the overall costs. The second part is that CubeSat programs only pays the engineering cost of the actual integration if they do not make the launch schedule. This establishes accountability for the universities, and in some cases justifies the many late nights in the lab working on a satellite that is about to be delivered.

The following list shows a summary of the ELaNa missions, the number of CubeSats that were manifested and the status of the missions. 


\section{INTRODUCTION}

\begin{tabular}{|l|c|c|l|}
\hline Mission & Primary Payload & Number of University CubeSats & Status \\
\hline ELaNa I & GLORY & 3 & $\begin{array}{l}\text { The launch vehicle failed to reach orbit. The P-POD success- } \\
\text { fully protected the primary payload and functioned as antic- } \\
\text { ipated. }\end{array}$ \\
\hline ELaNa II & ORS Enabler & 2 & $\begin{array}{l}\text { CubeSat integration postponed and the CubeSats re- } \\
\text { manifested on other ELaNa missions. }\end{array}$ \\
\hline ELaNa III & NPP & 5 & $\begin{array}{l}\text { Rocket successfully reached orbit and all of the satellite were } \\
\text { able to perform their missions. }\end{array}$ \\
\hline ELaNa IV & N/A & 7 & CubeSats manifested, unsure of orbit or mission. \\
\hline ELaNa V & N/A & 3 & $\begin{array}{l}\text { CubeSats manifested, unsure of orbit or mission. } \\
\text { ELaNa VI }\end{array}$ \\
NROL-36 & 4 & $\begin{array}{l}\text { All these CubeSats are slotted to be manifested but do not } \\
\text { have a mission yet. }\end{array}$ \\
\hline ELaNa VII, VIII & N/A & 46 &
\end{tabular}

Table 2.1: ELaNa Program Summary

\subsection{CubeSat Communications}

This section explains a brief history of amateur radio, how ground station networks have developed, and one of the popular formats for packet transmission in the CubeSat community. This section will also discuss two different types of CubeSat ground station networks. decibels (dBs) are frequently referred to when discussing electronics. A dB is a base ten logarithmic unit that describes the ratio of a physical quantity (such as the power of an electromagnetic wave) relative to a specificed or implied reference level [3]. An example of a decibel used in this thesis is a dBm, which is a unit based on a reference level of one milliwatt.

\subsubsection{Amateur Frequencies}

Congress passed the first laws regulating radio transmissions and creating the amateur radio community in 1912 [16]. As the amateur radio community grew, organizations such as the International Amateur Radio Union (IARU) and the Federal Communication Commission (FCC) built guidelines and procedures for amateur radio operators to follow for fair use of the radio spectrum [17]. The first Amateur Radio satellite Orbital Satellite Carrying Amateur Radio (OSCAR) was built in 1961. [18]. OSCAR 1, 
and the many satellites that followed, paved the way for the use of amateur radio frequencies in space. Many of the past and present CubeSats use the amateur frequencies pioneered by these satellites. As the amateur satellite radio community expanded, rules were established to maintain the success of the future amateur radio satellites. These include having a "turn off transmitter" signal that can be sent from the ground station, and that an individual licensed amateur radio operator without pecuniary interest [19] must control each satellite.This disqualifies some CubeSats with industry backers or financiall interest in the CubeSat's development from using the amateur spectrum. An example of one such satellite would be CSTB-1 [13]. A full list of requirements and procedures for applying for an amateur frequency is located in $\mathrm{A}$.

\subsubsection{Amateur Radio Ground Stations}

With satellites operating on the amateur frequencies, amateur ground stations needed to be established. As the amateur radio community grew, the amateur radio ground stations adapted to meet the needs of the next generation of CubeSats. When the CubeSat community first began, single stations were set up to command and downlink the data from each of the satellites in space. However, with a total of 30 minutes a day to talk to the satellite over a particular ground station the amount of data that can be downlinked was limited. One of the first CubeSat teams to overcome the single ground station limitation was the CubeSat team at Nakasuka Labs in Nakasuka, Japan. Using a decoder, they were able to use amateur ground stations around the world to listen and collect data, dramatically increasing the amount of data that could be collected from XI-V [20]. Many 


\section{INTRODUCTION}

teams have further developed this idea, such as the PolySat team and the

CP6 development team. They developed a software package called Massive

Operations, Recording and Experimentation Database System (More dBs)

[5]. This has led many other CubeSat teams to use distributed networks.

One great example of an expanded ground station network is shown below.

This is an example of the access available to the RAX-2 satellite [21].

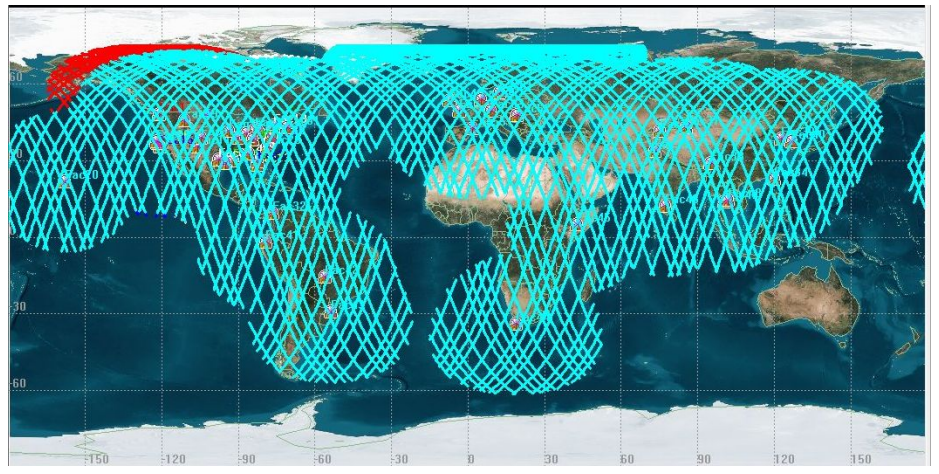

Figure 2.1: Coverage area of the RAX-2 ground station network. 


\subsubsection{AX.25}

One of the major contributors to the growth of amateur radio, as well as one of the largest limiting factors on complexity of the amateur radio community, is the AX.25 packet format. It has become popular in recent years because of the simple architecture and the use of the Automatic Packet Reporting System (APRS) [1]. These two aspects have led many radio suppliers to develop inexpensive, low power transmitters that are perfect for many university satellite projects. Although these low power transmitters are very popular, the major limiting factor is the large amount of overhead that is part of an AX.25 packet. Practical development has been limited to 9600 baud rate because of the large amount of data overhead. Another component of the AX.25 packets is a packet size limit of 256 Bytes [2]. A good explanation of the packet format and how transmission works can be read in reference [22].

\subsubsection{Global Educational Network for Satellite Oper- ations (GENSO)}

One constraint presented by using amateur radio operators around the world, is that there is still only one place to command the CubeSat satellite. Fortunately, there are a couple ways to overcome this issue. One possible solution is to open uplink commands to the entire amateur radio community. However, this could be a problem with complicated set of operations, or if the user simply wants positive control of the satellite. Another solution is having dedicated ground stations around the world. However, the communication between the ground stations could be an issue. In re- 


\section{INTRODUCTION}

sponse to this issue, the European Space Agency (ESA) is using the idea of dedicated ground stations without the individual investment to create a solution. The solution is called Global Educational Network for Satellite Operations (GENSO) [23]. GENSO allows a user to schedule time on remote servers, and command their satellite using the integrated network of servers around the world and existing ground stations. One major aspect or assumption was that GENSO was going to be a viable option for future CubeSat Missions; however at this time, the GENSO project is currently in an unusable state. For this reason, this thesis will not recommend the use of GENSO.

\subsubsection{Federated Ground Station Networks}

James Cutler of University of Michigan first mentioned the idea of the Federated Ground Station Network. In A Federated Ground Station Network, he presented the idea of using a loose collection of ground stations around the world to increase network access to space-borne assets[24]. He talks about virtualization of collections of ground stations and being able to utilize the large amount of potential ground stations. This idea is the major cornerstones of this paper and has been embraced by many CubeSat developers around the world. The federated ground station networks are meant to consist of autonomous, globally distributed ground installations.[24] The simplest form of this kind of network is the use of the amateur radio community. Although this network may not be completely autonomous, much of the work can appear to be autonomous to the CubeSat developer if the satellite decoder is written in a robust manner. 


\subsubsection{Downlinking Methods}

In chapter 5 , there is a comparsion of two methods for downlinking data. The two methods are a BC method and a GSI method.

\section{Broadcast (BC) Method of Downlinking}

BC method consists of continous transmission over the period of operation. This method is going to be extremely power intensive and can not be used if there are any transmission restrictions.

\section{Ground Station (GSI) Initiated Downlinking}

GSI downlinking requires the main ground station to initiate the downlinking. While this method will be less power intensive, there may be a decrease in the downlinking capabilites since some ground stations may not be utilitized. This method also allows a user to tailor the downlinking period to maximize the transmit time. 


\section{INTRODUCTION}

\section{$2.3 \quad$ STK}

The Satellite Tool Kit is a general-purpose modeling and analysis application for any type of space, defense, or intelligence system [7]. AGI developed STK in 1989 as an orbital analysis and access calculator. It is built on AGI's patented spatial mechanics engine and integrated visualization. In its base form, STK addresses a majority of the requirements for concept development and preliminary system or mission designs [7]. The STK tools model many systems including battlefield simulations, or missile defense systems. This thesis uses STK to model satellites, their orbits, and the ground coverage times extensively.

This thesis uses STK to calculate access times for many ground stations, orbital and frequency options. This is done by using the interconnection commands provided by the connect module of STK with MATLAB . This will allow STK to run the models of the desired system and then output the access reports to MATLAB for further processing. Then brute force optimization will be used to find the best orbit or ground station configuration for a desired mission. In the current version of CMPT, CMPT can only use STK to calculate the access. This may change in future releases.

\subsubsection{Interfacing}

There are two ways to interface with STK. The easiest way is to use STK as a standalone program. The other way used by CMPT uses STK through the "connect" commands. This section discusses how to set up and use STK for CMPT, and the code used to initialize the connection between 
STK and MATLAB .

\subsubsection{Scenarios}

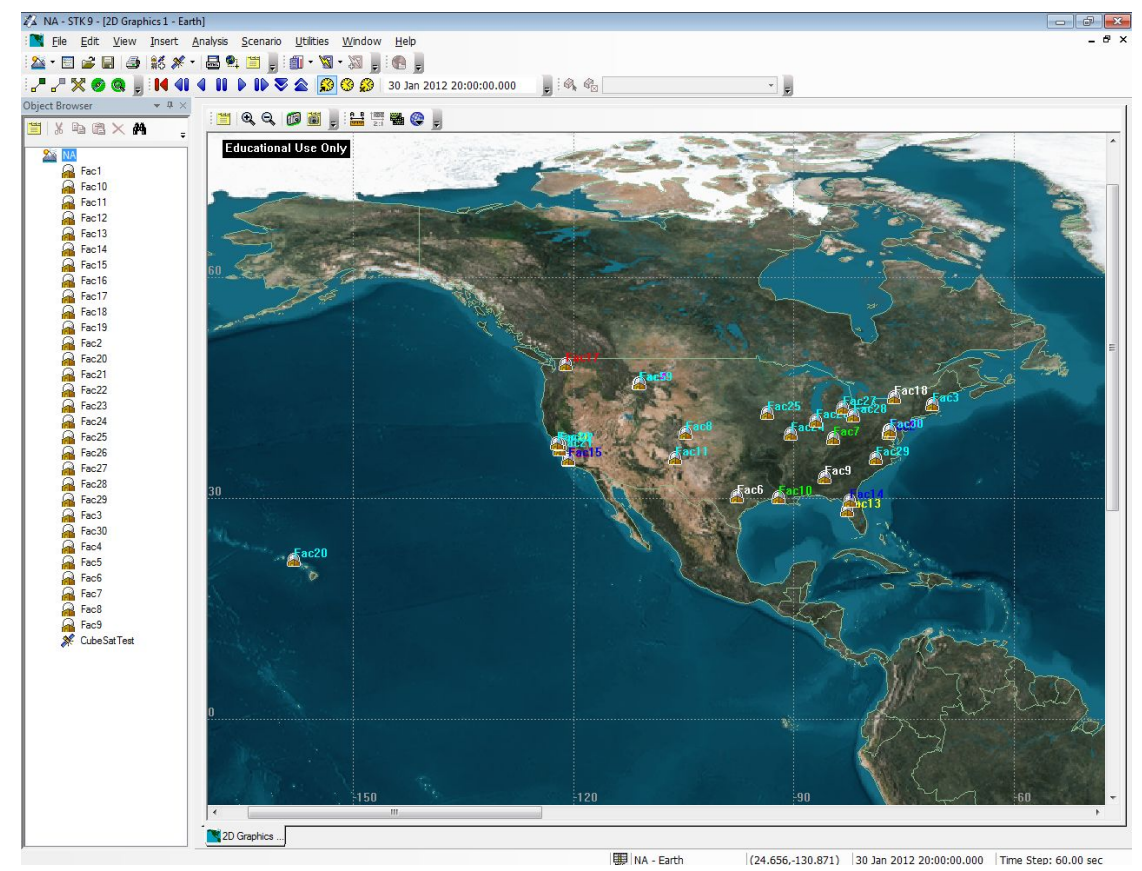

Figure 2.2: Screenshot of STK and the North America Scenario.

To start any STK modeling simulation, the first thing that needs to be set up is a scenario. A scenario consists of a couple major components such as satellites, transmitters, and ground facilities.For increased efficiency, it is important to add all the ground facilities used over the course of the simulation in the initial set up of STK. During the testing and simulations, adding components such as satellites, transmitters, or facilities requires more time to execute than modifying the existing objects of a scenario.

\subsubsection{Objects}

Objects such as facilities, satellites or vehicles are available in the object options window. The object options window has many properties for any 


\section{INTRODUCTION}

of these objects including physical location, velocities, and masses.

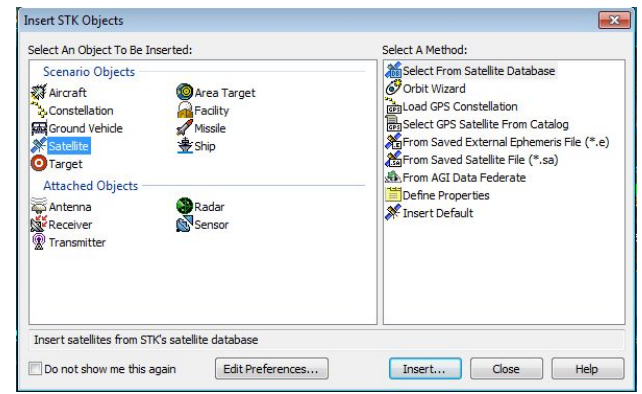

Figure 2.3: Screenshot of the Object options of STK. 


\subsubsection{Access Reports}

STK uses accurate projections to predict when and where a satellite or other object will interact with facilities or other areas of interest on the ground. The graphical representation of the access reports are a few button commands in the STK viewer. As seen in the figure below, STK makes it easy to see the area of coverage, and the amount of time the satellite passes over a facility.

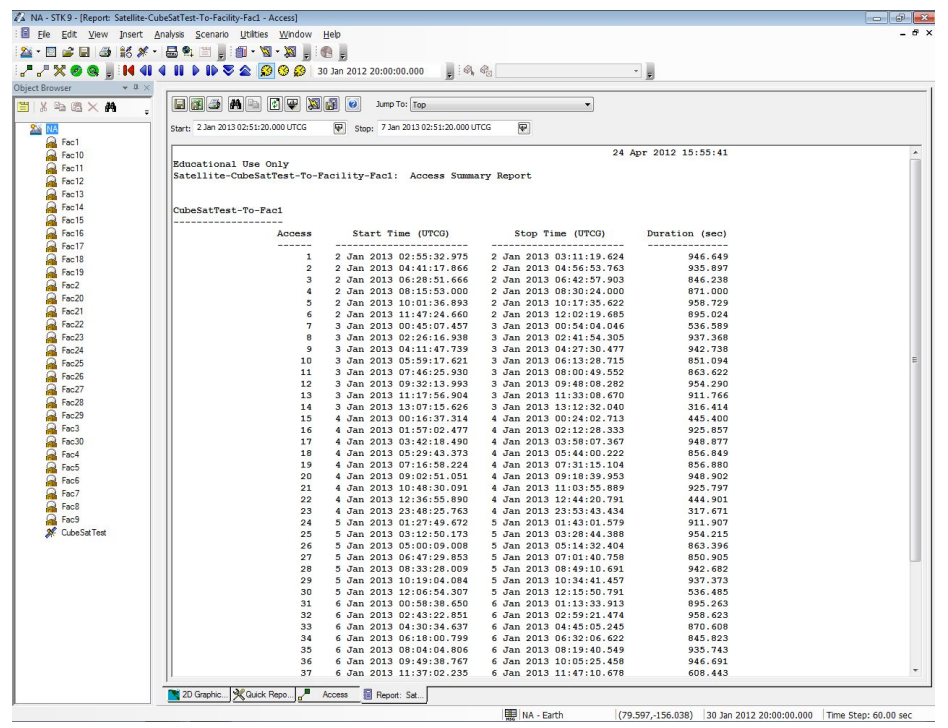

Figure 2.4: Access Report in STK.

\subsubsection{Interoperability and Integration Module (IIM)}

The Professional STK suite has more modules than are used in this thesis, and these modules simulate anything from battlefields to orbital debris. The major module used in this thesis is the Interoperability and Integration module (IIM) which allows STK to interface with most scripting languages. [7] The IIM allows for the use of the connect commands described earlier and makes this thesis possible. Online support is available to help with 


\section{INTRODUCTION}

the connect commands, which are usable with C, MATLAB , or TCP/IP socket interfacing. There are examples in the code on different connect commands, however, the most important ones are the initiation commands can be found in section 2.3.6.

\subsubsection{Connect Commands}

The code below initiates the connection between MATLAB and STK.

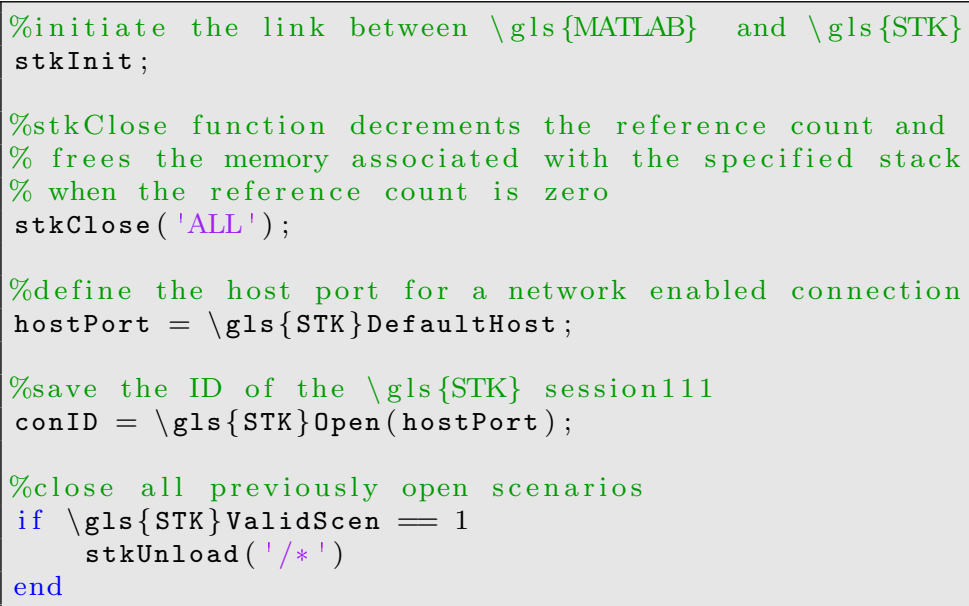

Figure 2.5: STK Initialization MATLAB code

The code below opens scenarios using load commands. The stkExec function call is the cornerstone of any program used to interface with STK and MATLAB .

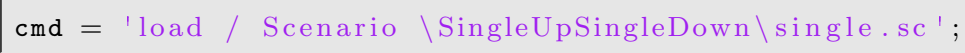

Figure 2.6: MATLAB code to load scenario

The code below shows how to add a new object to a scenario such as a 
satellite.

stkNewObj ( '*/', ' Satellite ', ' CubeSatTest ') ;

Figure 2.7: MATLAB code to add object

Although the commands to initiate an access report and consequently pull the information of the report back into MATLAB are easy to use, getting the report into a useable format requires a couple tricks that can be observed in apendix D.2. 


\section{3}

\section{CubeSat Survey}

Preceding the work to develop the CMPT, a survey of past CubeSat missions was put together to gain a better understanding of past CubeSat trends. These trends helped to simplify mission planning for CubeSat systems engineers and mission planners. To accomplish this trending and organize the past missions, a matrix was created with each of the CubeSat missions. This includes the mission type, the university or organization responsible, mission length (if successful), amount of data collected, frequency used, power transmitted (if available) and the orbit. The CubeSat Survey Matrix is located in B

\subsection{Research}

The research for the CubeSat Survey was accomplished with the assistance of CubeSat: a Review [25], A Survey of CubeSat Communication Systems [9], a previous CubeSat matrix and quite a few conversations with CubeSat developers. However, since many of the early CubeSat missions were low budget and student run programs, information about the specifics of the 
systems was not accessible. 


\subsection{Mission Success and Failure}

The definition of success varies widely between CubeSat missions. For some CubeSats, success is defined by getting to the launch pad and proving their university can build a flight satellite. However, success can be as complicated as verifying through optical systems the production of proteins from genetic activity such as GeneSat [26]. One of the interesting characteristics of the past successful missions is that if communications were established then the rate of success is dramatically higher, even for the more complicated missions. Therefore it can be concluded that a successful mission is a mission that establishes a communication link.

\subsection{Defining Characteristics of Past Missions}

As the CubeSat matrix was constructed, the defining characteristics became apparent. The aspects considered for each mission types are planned mission length, size, orbit, mission description, data rate, and power out of transmitter.

\subsubsection{Planned Mission Length}

Planned mission length is a determining factor of any CubeSat mission. This determines many characteristics of the CubeSat including battery sizes, data storage and ultimately the amount of data downlink capability. Planned mission length can range from one month to multiple years. 


\subsubsection{Size}

Size is the volume of the CubeSat. The size of a CubeSat directly affects the complexity of a mission; science missions will generally have much more complex payloads than that of an initial attempt satellite. The greater the size of the satellite, the larger the batteries that can be accommodated, and consequently higher transmit power and data rates. The sizes in past missions are $1 \mathrm{U}, 2 \mathrm{U}$ and $3 \mathrm{U}$.

\subsubsection{Orbit}

While all of the past missions, regardless of type, have been in the low earth orbit (LEO) orbit, missions in the future may have a variety of orbital options from extreme LEO to geosynchronous orbits, and beyond. Although orbital parameters are a characteristic of a mission type, orbital parameters do not contribute to determining mission type since most CubeSat missions have been in the same $600 \mathrm{~km}$ to $800 \mathrm{~km}$ altitude orbit. For example, initial attempt satellites will generally only occupy the LEO. While the science missions will have opportunities in the medium earth orbit (MEO) and geosynchronous (GEO) orbits in the not so far future. The main reason initial attempt satellites are limited to LEO is because of the limited transmitter power. If a CubeSat wanted to maintain communication with a satellite in MEO, it would require far more than the one watt transmitter typical of a initial attempt mission. 


\subsubsection{Mission Description}

The description of the mission can be very beneficial when trying to understand the type of mission and the complexity of the system. This parameter can be the quickest way to develop a rubric for comparison. Key words and concepts used in the mission are helpful in determining type of missions.

An example of this is the mission description of RAX-2.

To study FAI, the RAX mission will utilize large incoherent scatter radar in Poker Flats, Alaska (known as PFISR). PFISR will transmit powerful radio signals into the plasma instabilities that will be scattered into space. During that time, the RAX spacecraft will be orbiting overhead and recording the scatter signals with an onboard receiver. These signal recordings will be processed by an onboard computer and transmitted back to our ground stations where scientists will analyze them. The goal of this one-year science mission is to enhance our understanding of FAI formation so that short-term forecast models can be generated. This will aid spacecraft operators with planning their mission operations around periods of expected communication disruption. It can be seen from this description that an extremely large amount of data is going to be produced, that the mission length will last one year and that this mission will potentially be very power heavy and will require a $3 \mathrm{U}$ form factor. $[27]$

Some of the key phrases found in this mission description are the one year science mission, extremely large amount of data, and the very power heavy statements. As well as the description of the science that will lead a person to think this mission will be a complex science mission.

\subsubsection{Data Rate}

A good indicator of mission type is the downlink data rate, since the amount of data a cubesat can downlink is a huge limiting factor to the capabilities of a mission. Initial attempt missions can reduce the complexity by limiting 


\section{CUBESAT SURVEY}

their data rate to 1200 bits per seconds (bps) and still be able to accomplish their mission. However, science missions require much higher data rates to downlink the higher amount of payload data. A typically data rate for the science missions is $9600 \mathrm{bps}$.

\subsubsection{Transmitter Power}

One of the major limiting factors of a satellite is its ability to communicate with the ground and as a result the transmit power can be an extremely useful indicator of the type of mission. Initial attempt missions will generally have a transmitter power lower than one watt, while science or test-bed missions may transmit as high as 2.5 watts. 


\subsection{Categories}

This section explains each of the four mission types. This section also includes some examples of each, and the parameters that CMPT will use when calculating suggestions for the CubeSat Satellite developer. After looking at a number of CubeSat missions, four distinct groups of mission types were evident.

\subsubsection{Initial Attempts}

Initial attempts are simple missions with minimal requirements and constraints on power, communications and budgets. CP1 [28], BeeSat [29] and SwissCube [30] are examples of Initial Attempt missions. All of these missions and most the other initial attempt satellites were $1 \mathrm{U}, 430$ megahertz $(\mathrm{MHz})$ satellites, with either basic batteries or running solely on solar cells. As a result, the amount of data collected could fit on a handful of floppy disks.

CMPT uses the following parameters for Initial Attempt satellites:

\subsubsection{One-Shot}

A One-shot satellite is a mission that has one major event such as a deployable or major event. One-Shot satellites are not as abundant as other types of satellites such as Initial Attempt or Science missions, however is an important category to focus attention to when looking at the overall trends of CubeSat missions. In an opening presentation at the small Satellite Conference in Logan, Utah, an Army general mentioned the need for responsive missions: where missions are ready for launch in days, not years. It could 


\section{CUBESAT SURVEY}

\begin{tabular}{|l|c|}
\hline Parameter & Value \\
\hline Frequency (4.1.5) & $430 \mathrm{MHz}$ \\
Bus Voltage (4.1.5) & $3.3 \mathrm{~V}$ \\
Battery Density (4.1.5) & 180 days \\
Mission Length (4.1.5) & 3 megabyte (MB) \\
Total Data Produced (4.1.6) & 1 day \\
Data Period (4.1.6) & $1 \mathrm{watt}$ \\
Max Allowable Power (4.1.7) & $1200 \mathrm{bps}$ \\
Data Rate (4.1.7) & $50 \%$ \\
Transmitter Duty Cycle (4.1.7) & $5 \mathrm{dBm}$ \\
Spacecraft Antenna Gain (4.1.7) & $16.1 \mathrm{dBm}$ \\
Ground Station Antenna Gain $(4.1 .7)$ & $2 \mathrm{~dB}$ \\
Pointing Losses (4.1.7) & $15 \mathrm{dBm}$ \\
Desired $E_{b} / N_{0}(4.1 .7)$ & $542 \mathrm{kelvin}(\mathrm{K})$ \\
Noise Temperature (4.1.7) & \\
\hline
\end{tabular}

Table 3.1: Parameters for Initial Attempt satellites

be argued that some of these missions would be One-Shot satellites, taking images of a particular geographical region or event.

CMPT uses the following parameters for One-Shot satellites: 


\begin{tabular}{|l|c|}
\hline Parameter & Value \\
\hline Frequency (4.1.5) & $430 \mathrm{MHz}$ \\
Bus Voltage (4.1.5) & $3.3 \mathrm{~V}$ \\
Battery Density (4.1.5) & $1200 \mathrm{mAh}, 1840 \mathrm{mAh}$ \\
Mission Length (4.1.5) & 180 days \\
Total Data Produced (4.1.6) & $90 \mathrm{~dB}$ \\
Data Period (4.1.6) & $1 \mathrm{watt}$ \\
Max Allowable Power (4.1.7) & $1200 \mathrm{bps}$ \\
Data Rate (4.1.7) & $50 \%$ \\
Transmitter Duty Cycle (4.1.7) & $5 \mathrm{dBm}$ \\
Spacecraft Antenna Gain (4.1.7) & $16.1 \mathrm{dBm}$ \\
Ground Station Antenna Gain $(4.1 .7)$ & $2 \mathrm{~dB}$ \\
Pointing Losses (4.1.7) & $15 \mathrm{dBm}$ \\
Desired $E_{b} / N_{0}$ (4.1.7) & $542 \mathrm{~K}$ \\
Noise Temperature (4.1.7) & \\
\hline
\end{tabular}

Table 3.2: Parameters for One-Shot satellites

\subsubsection{Science Missions}

The Science mission category are missions developed to test a particular anomaly or situation in orbit. This could be to study large plasma formations in the Ionosphere [27] with RAX 1 and 2, or GeneSat-1's onboard micro-laboratory experiments that could detect proteins in bacteria that are products of specific genetic activity [26]. Most of these missions are using a $3 \mathrm{u}$ form factor and utilize more frequencies than just the $430 \mathrm{MHz}$ range to facilitate larger bandwidths. Most science missions have mission lives of one year or longer and have higher data rates.

CMPT uses the following parameters for Science satellites: 


\section{CUBESAT SURVEY}

\begin{tabular}{|l|c|}
\hline Parameter & Value \\
\hline Frequency (4.1.5) & $530 \mathrm{MHz}, 900 \mathrm{MHz}, 2.4$ gigahertz $(\mathrm{GHz})$ \\
Bus Voltage (4.1.5) & All \\
Battery Density (4.1.5) & 365 days \\
Mission Length (4.1.5) & $100 \mathrm{MB}$ \\
Total Data Produced (4.1.6) & 2 day \\
Data Period (4.1.6) & $2 \mathrm{watt}$ \\
Max Allowable Power (4.1.7) & $2400,9600 \mathrm{bps}$ \\
Data Rate (4.1.7) & $50 \%$ \\
Transmitter Duty Cycle (4.1.7) & $27 \mathrm{dBm}$ \\
Spacecraft Antenna Gain (4.1.7) & $16.1 \mathrm{dBm}$ \\
Ground Station Antenna Gain $(4.1 .7)$ & $3 \mathrm{~dB}$ \\
Pointing Losses (4.1.7) & $15 \mathrm{dBm}$ \\
Desired $E_{b} / N_{0}(4.1 .7)$ & $542 \mathrm{~K}$ \\
Noise Temperature (4.1.7) & \\
\hline
\end{tabular}

Table 3.3: Parameters for Science satellites

\subsubsection{Test-Beds}

Test-Bed missions are corporate backed satellite than generally cannot operate on an amateur frequency band. However, the budgets for these satellites are often much higher than that of university-funded satellites. They range from the $1 \mathrm{U}$ form factor like the CSTB-1 [13] or a $3 \mathrm{U}$ form factor satellite similar to LightSail-1 [31].

CMPT uses the following parameters for Test-Bed satellites:

\subsubsection{Custom}

As mission development continues, the CubeSat's specifications will become more concise and realistic in terms of creatability. The custom category is designated for the missions with unique characteristics or further along in the design process. All of the values are open for adjustment. 


\subsection{Categories}

\begin{tabular}{|l|c|}
\hline Parameter & Value \\
\hline Frequency (sec 4.1.5) & $430 \mathrm{MHz}, 900 \mathrm{MHz}, 2.4 \mathrm{GHz}$ \\
Bus Voltage (sec 4.1.5) & All \\
Battery Density (sec 4.1.5) & 365 days \\
Mission Length (sec 4.1.5) & $100 \mathrm{MB}$ \\
Total Data Produced (sec 4.1.6) & $2 \mathrm{day}$ \\
Data Period (sec 4.1.6) & $2 \mathrm{watt}$ \\
Max Allowable Power (sec 4.1.7) & $2400,9600 \mathrm{bps}$ \\
Data Rate (sec 4.1.7) & $50 \%$ \\
Transmitter Duty Cycle (sec 4.1.7) & $27 \mathrm{dBm}$ \\
Spacecraft Antenna Gain (sec 4.1.7) & $16.1 \mathrm{dBm}$ \\
Ground Station Antenna Gain $(\sec 4.1 .7)$ & $3 \mathrm{~dB}$ \\
Pointing Losses (sec 4.1.7) & $15 \mathrm{dBm}$ \\
Desired $E_{b} / N_{0}(\sec 4.1 .7)$ & $542 \mathrm{~K}$ \\
Noise Temperature (sec 4.1.7) & \\
\hline
\end{tabular}

Table 3.4: Parameters for Test-Bed satellites

\subsubsection{Matrix of Mission Parameters}

The table below is a summary of the missions and their parameters.

\begin{tabular}{|c|c|c|c|c|c|}
\hline Parameters & Initial & One-Shot & Science & Test-Bed & Custom \\
\hline Orbit (sec 4.1.4) & LEO & LEO & LEO MEO & LEO MEO & All \\
\hline Ground Station (sec 4.1.3) & NA FW SS & NA FW SS & NA FW SS & NA SS & All \\
\hline \multicolumn{6}{|c|}{ Satellite Parameters } \\
\hline Frequency (sec 4.1.5) & $430 \mathrm{MHz}$ & $430 \mathrm{MHz}$ & $2.4 \mathrm{GHz}$ & All & All \\
\hline Bus Voltage ( $\sec 4.1 .5)$ & 3.3 & 3.3 & 5 & All & All \\
\hline Battery Options (sec 4.1.5) & $1200 \mathrm{mAh}, 1840 \mathrm{mAh}$ & $1200 \mathrm{mAh}, 1840 \mathrm{mAh}$ & All & All & All \\
\hline Mission Length ( $\sec 4.1 .5)$ & 180 days & 90 days & 365 days & 365 days & All \\
\hline \multicolumn{6}{|c|}{ Data Parameters } \\
\hline Peak Data Produced ( $\sec 4.1 .6)$ & - & - & - & - & All \\
\hline Period of Generation ( $\sec 4.1 .6)$ & - & - & - & - & All \\
\hline Total Data Produced (sec 4.1.6) & $3 \mathrm{MB}$ & $10 \mathrm{MB}$ & $100 \mathrm{MB}$ & $100 \mathrm{MB}$ & All \\
\hline Data Period (sec 4.1.6) & 1 day & 90 days & 2 days & 2 days & All \\
\hline \multicolumn{6}{|c|}{ Transmitter Parameters } \\
\hline Max Allowable Power (sec 4.1.7) & 1 watt & 1 watt & 2 watt & 2 watt & All \\
\hline Downlink Data Rate (sec 4.1.7) & $1200 \mathrm{bps}$ & $1200 \mathrm{bps}$ & $9600 \mathrm{bps}$ & $9600 \mathrm{bps}$ & All \\
\hline Duty Cycle (sec 4.1.7) & $50 \%$ & $50 \%$ & $20 \%$ & $20 \%$ & All \\
\hline Spacecraft Antenna Gain (sec 4.1.7) & $5 \mathrm{~dB}$ & $5 \mathrm{~dB}$ & $27 \mathrm{~dB}$ & $27 \mathrm{~dB}$ & All \\
\hline Ground Station Antenna Gain ( $\sec 4.1 .7)$ & $16.1 \mathrm{dBm}$ & $16.1 \mathrm{dBm}$ & $36 \mathrm{dBm}$ & $36 \mathrm{dBm}$ & All \\
\hline Pointing Losses (sec 4.1.7) & $2 \mathrm{~dB}$ & $2 \mathrm{~dB}$ & $3 \mathrm{~dB}$ & $3 \mathrm{~dB}$ & All \\
\hline Desired $E_{b} / N_{0}(\sec 4.1 .7)$ & $15 \mathrm{dBm}$ & $15 \mathrm{dBm}$ & $15 \mathrm{dBm}$ & $15 \mathrm{dBm}$ & All \\
\hline Noise Temperature (sec 4.1.7) & $542 \mathrm{~K}$ & $542 \mathrm{~K}$ & $542 \mathrm{~K}$ & $542 \mathrm{~K}$ & All \\
\hline Line Losses (sec 4.1.7) & $1 \mathrm{~dB}$ & $1 \mathrm{~dB}$ & $1 \mathrm{~dB}$ & $1 \mathrm{~dB}$ & All \\
\hline Downlink Losses (sec 4.1.7) & $4.6 \mathrm{~dB}$ & $4.6 \mathrm{~dB}$ & $4.6 \mathrm{~dB}$ & $4.6 \mathrm{~dB}$ & All \\
\hline
\end{tabular}

Table 3.5: Matrix of the parameters for each of the satellite categories 


\section{4}

\section{CubeSat Mission Planning}

\section{Toolbox}

The CubeSat Mission Planning Toolbox (CMPT) is a MATLAB interface

allowing a user to select satellite parameters and simulate operations using STK. CMPT is set up in three main parts. CMPT initially opens the setup screen(fig 4.1) where the satellite parameters are set. The next window is the summary screen(fig 4.12) where calculations and simulations are accomplished. Finally, the results screen(fig 4.19) presenting users with parameter suggestions and allows users to print out graphs and data.

\subsection{CMPT Setup}

\subsubsection{Mission Type}

Mission types allow for quick use of the program through pre-allocated parameters that are shown in table 3.5. 


\section{CUBESAT MISSION PLANNING TOOLBOX}

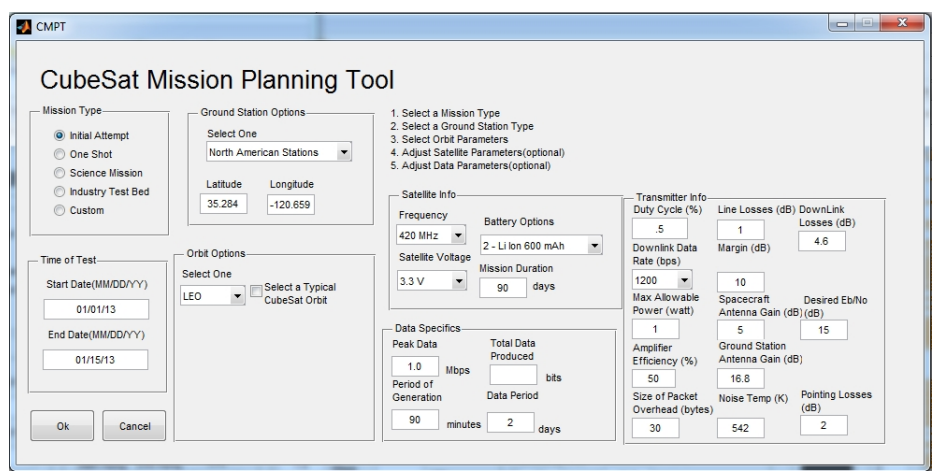

Figure 4.1: Screenshot of the CubeSat Mission Planning Toolbox Setup Window.

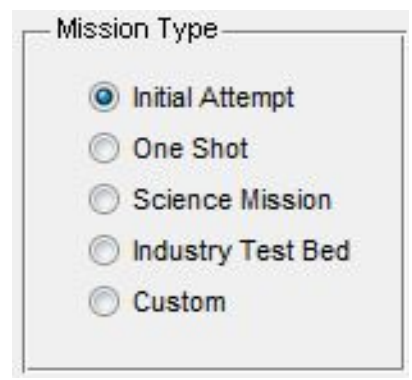

Figure 4.2: Close up of the Mission Type Panel

Mission Type Option Descriptions:

- Initial Attempt CubeSats are generally an organization's first attempt at a satellite. The Initial Attempt CubeSats generally have low power transmitters, short mission lives and smaller budgets. Section 3.4.1 explains mission type in detail.

- One-Shot CubeSats are missions that focuses on a single events, where most of the data collection occurs, and then the rest of the mission is used for data downlink. These missions generally have shorter mission lives and lower transmit power and potentially larger amounts of data produced than Initial Attempt Satellites. Section 3.4.2 explains mission type in detail.

- Science CubeSats are missions with complicated scientific opera- 
tions, characterized by longer mission lives, and larger budgets. Section 3.4.3 explains mission type in detail.

- Test Bed CubeSats are corporate sponsored satellite that will generally have higher budgets. Section 3.4.4 explains mission type in detail.

- Custom CubeSats allow all the parameters to be changed in CMPT such as the Max Allowable Power (MAP)or the downlink data rate(sec 4.1.7). Section 3.4.5 explains mission type in detail.

\subsubsection{Time of Test}

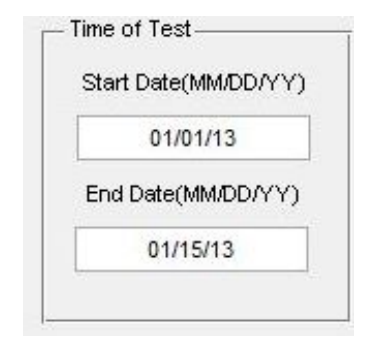

Figure 4.3: Close up of the Time of Test Panel

The time of test parameter allows a user to set when and how long a test will take place. This parameter allows the user to select a time period from Jan. 1, 2012 to anytime in the future. CMPT will not allow you to input dates that are out of order. CMPT may also change the test period based on test parameters. CMPT uses Coordinated Universal Time (UTC) time for the simulations and display. 


\subsubsection{Ground Stations Options}

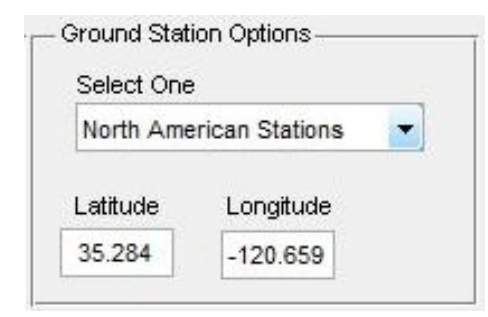

Figure 4.4: Close up of the Ground Station Panel

The ground station option allows a user to select North American based ground stations, ground stations around the world, or a single ground station. The longitude and latitude of the main ground station can be changed in the textbox provided.

As decoders and distributed data collection grew in popularity, so did the amount of ground stations available to downlink satellite data that use the amateur radio frequencies. There are many stations around the world and constant coverage could give an operator unlimited downlink opportunities. However, there are times when ground stations options are limited or simply not allowed. The use of modulation schemes such as Binary Phase Shift Keying (BPSK) can limit the satellite's downlinking opportunities because the typical ground station can not demodulated the incoming transmission. Other satellites are not allowed to use distributed ground stations, nor transmit anywhere besides over the continental United States of America when they have an experimental FCC license, which not only limits the transmitting capabilities of the satellite, but also requires the satellites mission life to 6 months. It is also important to note that experimental frequencies do not allow the use of beacons. Beacons are helpful periodic transmissions used to acquire satellite health and status information. 
Note: A future revision of the program may allow for analyzing individual ground stations to better utilize the network available. CMPT assumes that all ground stations are available 24 hours 7 days a week.

Ground Station Option Descriptions:

- North America Ground Stations

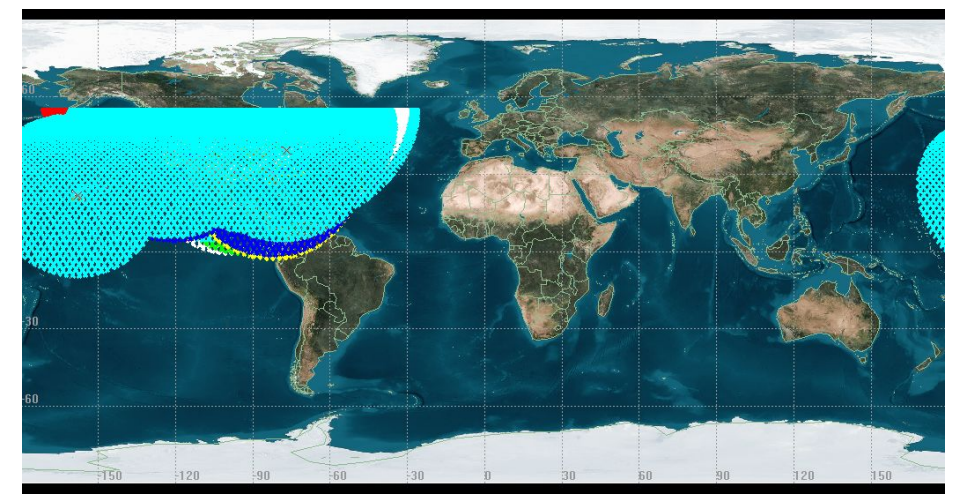

Figure 4.5: A screenshot from STK representing the coverage area of the North American Ground Station network.

The More dBs network defined the North American ground stations. Figure 4.5 shows the network available. The North American ground station network focuses on missions that are limited to North America such as CSTB-1 [13].

- Full World Ground stations

The RAX-2 network was the model for the Full World ground station network. The most up to date RAX-2 network is on their shared Google maps page [32]. This option is not available to the Test bed missions (3.4.4) as they often have some sort of profit associated with the product being tested. As mentioned in section 2.2.1 there can be 


\section{CUBESAT MISSION PLANNING TOOLBOX}

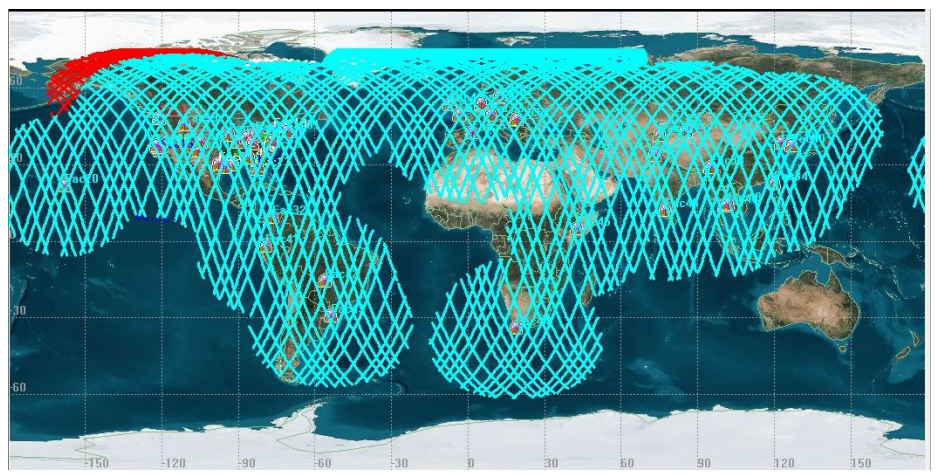

Figure 4.6: Coverage area of the RAX-2 ground station network.

no profit gained from the work accomplished and transmitted over the amateur radio frequencies.

- Single Ground Station

When developing a specialized modulation scheme or unique frequency allocation there us a need for a single station to analyze and potentially compare with other ground station option.

\subsubsection{Orbital Options}

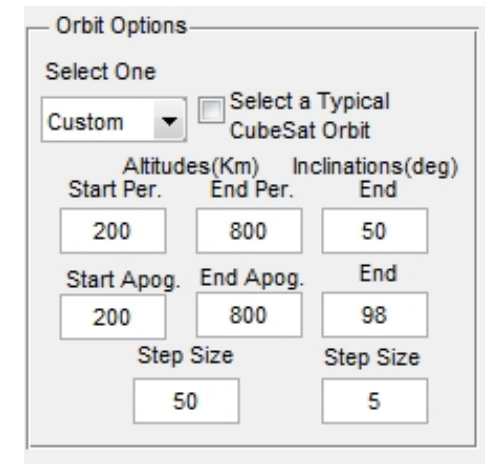

Figure 4.7: Close up of the Orbits Option Panel

When looking at the orbital characteristics of the past CubeSat missions, the $600 \mathrm{~km}$ to $800 \mathrm{~km}$ altitude range is a CubeSat sweet spot. While most of these missions had little choice about their orbit, the ELaNa pro- 
gram has been aware that missions may have more specific needs.

If the "Typical cubeSat orbit checkbox" is checked, then the orbits options will be deselected and the orbit will be set to a $745 \mathrm{Km}$ circular orbit and an inclination of 98 degrees. This orbit was found by averaging all the past apogees. 


\section{CUBESAT MISSION PLANNING TOOLBOX}

Orbital Option Descriptions

\begin{tabular}{|l|c|c|}
\hline Orbit Option & Altitude Range & Inclination Range \\
\hline LEO & $100-2000 \mathrm{~km}$ circular orbits & $50-102$ degrees \\
MEO & $2000 \quad 35000 \mathrm{~km}$ circular orbits & 50102 degrees \\
Custom & Any & Any \\
Single Orbit & Any & Any \\
\hline
\end{tabular}

Table 4.1: Orbital Options description

Additional Notes:

Custom - Allows users to define the orbital range to meet a variety of needs.

CMPT will calculate the eccentricity steps to best accommodate the range desired.

Single orbit The option is used when the orbit is known and the user is trying to understand the ground station or satellite parameters. Singular orbits do not have to be circular.

\subsubsection{Satellite Information}

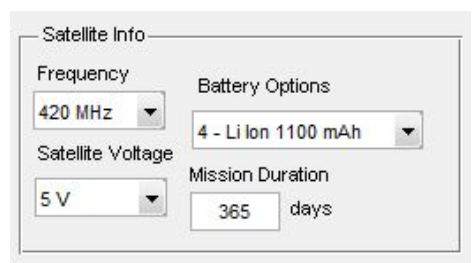

Figure 4.8: Close up of the Satellite Info panel

Satellite Information section provides information on the use of the inputs in the Satellite Information panel.

\section{Frequency}

Frequency allocation for a CubeSat satellite is limited to a few select frequencies from the available frequencies. While the program has all the 
amateur frequencies available, the most common frequencies are the 430 $\mathrm{MHz}, 900 \mathrm{MHz}$, and $2.4 \mathrm{GHz}$. As mentioned in the amateur radio requirements for use of the amateur radio frequencies, a satellite developer or any contributing body cannot be making money from the information over the amateur communication link [19]. Another major requirement is that all the information passed over the amateur radio frequencies must be freely accessible to the public through either publications or other such means [19]. Options are available to those that are making money or simply want to protect their data for scientific purposes. One option is to apply for an experimental license; this process is not particularly difficult but can take many months for the paperwork to route through the appropriate agencies. There are also similar processes in place for corporations to apply for individual licenses. For information on how to apply for an experimental license, please refer to https://apps.fcc.gov/oetcf/els/

Selectable frequency options

- $430 \mathrm{MHz}$

- $902 \mathrm{MHz}$

- $1240 \mathrm{MHz}$

- $2300 \mathrm{MHz}$

- $2390 \mathrm{MHz}$

- $3300 \mathrm{MHz}$

- $5650 \mathrm{MHz}$

- $10 \mathrm{GHz}$

- $24 \mathrm{GHz}$ 


\section{CUBESAT MISSION PLANNING TOOLBOX}

\section{Bus Voltage}

Smaller batteries and lower power generation capabilities typically limit the CubeSat's bus voltage. Although many CubeSats are limited to the 3V3 or $5 \mathrm{~V} 0$ bus voltage, the community and CubeSat parts suppliers have been increasing the product offerings and designing increasing more efficient components. The calculations of "current densities" and "percent of batteries used" utilize the bus voltage are located in section 4.4.5. All the values in the list below were chosen because of past or present missions using these bus voltages.

Bus voltage options

- $3 \mathrm{~V} 3$

- $5 \mathrm{~V} 0$

- $5 \mathrm{~V} 2$

- $6 \mathrm{~V} 0$

- $7 \mathrm{~V} 4$

\section{Batteries}

Batteries are the primary source of power for many CubeSat satellites. Many of the original CubeSat designed utilized off the shelf cell phone batteries such as Roses LIP-1S1P-1950 [33] which have been used on all flight proven PolySat satellites or Ultralifes UBP043048/PCM batteries used in Colorado Spaces Hermes Satellite [34]. Missions in development have been expanding the battery options by become more complex and require higher amp-hours (Ahs). The CubeSat developers have used custom battery packs such as those that will be used in LightSail-1 or higher end space rated batteries such as those flown in RAX 1 and 2 utilize 7V4 raw bus 
voltage and $4400 \mathrm{mAh}$ from CubeSatKit.com [35]. The selectable options are batteries available for purchase and have been used in past missions. Battery options:

\begin{tabular}{|c|l|l|}
\hline Number of Batteries & Type of Battery & Capacity of Each Battery \\
\hline 2 & Li Ion & $600 \mathrm{mAh}$ \\
2 & Li Ion & $920 \mathrm{mAh}$ \\
2 & Li Ion & $1276 \mathrm{mAh}$ \\
2 & Li Ion & $1950 \mathrm{mAh}$ \\
4 & Li Ion & $1100 \mathrm{mAh}$ \\
1 & Li Ion & $5800 \mathrm{mAh}$ \\
\hline
\end{tabular}

Table 4.2: Battery Options

\section{Planned Mission Length}

As described in the consideration section 3.3.1, the planned mission length is the time a developer planned for the satellite to finish its primary mission. Most CubeSats will be in orbit for more years than a CubeSat developer would need to complete their mission. Typically, planned mission lengths have been from three months to one year.

\subsubsection{Data Specific}

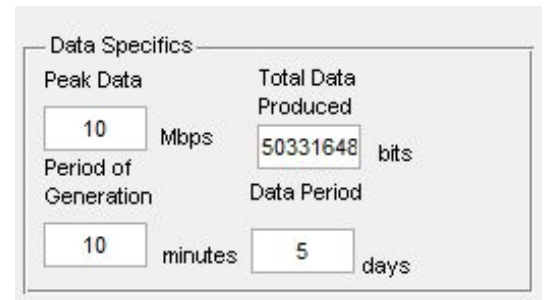

Figure 4.9: Close up of the Data Specifics Panel

The data specific parameters allow the user to input the data generation options of the payload. All generation is assumed to be instantaneous for 


\section{CUBESAT MISSION PLANNING TOOLBOX}

the sake of the calculations and the amount of time spent downlinking data will be larger than the time it requires to perform any of the experiments on CubeSat missions. Further the data that is being simulated during the experiments should be the form of the data as it is transmitted from the satellite. If the data is going to be compressed, the compressed values should be input into CMPT.

\section{Peak Data Produced}

When data collection is a stream or in the form of sensor data, the peak data produced is the maximum flow of data produced during an experiment. The peak data produced is measured in Megabits per second (Mbps).

\section{Period of Data Generation}

This parameter goes together with the peak data produced parameter and will generate a worst-case scenario for data production. It is important to note that this is not for the beacon data but, for the major data collection of experiments conducted during the satellite's mission life. The period of data generation is measured in minutes.

\section{Total Data Production}

Total Data Production is for missions that are taking pictures or have a fixed data size and are not generating periods of sensor data. An example of these missions is XI-IV, where the main missions are to get pictures in space [36]. However, this parameter is not limited to Initial Attempt missions(3.4.1). Rapid Terrestrial Imaging CubeSat Constellation (RTICC) is not an Initial Attempt(3.4.1) satellite, however the mission will generate 
up to 10 megapixel images that could reach 100 Megabits in size [37]. The total data production input is in bits.

\section{Data Period}

The data period parameter defines how much time passes between each experiment. Data period uses days and will determine the period of the test if the period is smaller than that of the original test period. This will determine the size of storage if the amount of data downlinked is less than that of the total data generated. The data period also contributes to the data storage equations in section 4.4.9.

\subsubsection{Transmitter Information}

The Transmitter Information section will explain the transmitter inputs.

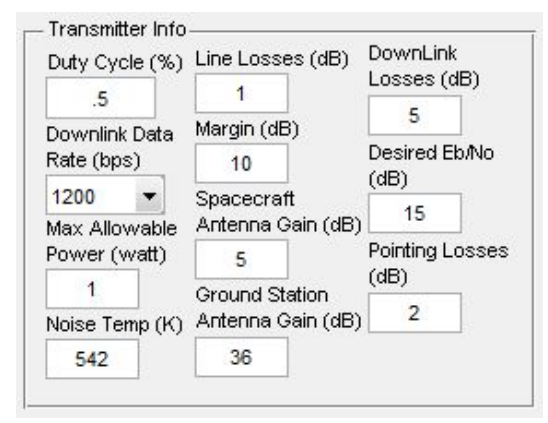

Figure 4.10: Close up of the Transmitter Info Panel

\section{Max Allowable Power (MAP)}

The MAP parameter will limit the orbit available to a CubeSat based on the link budget as described in section 4.4.5. The MAP is generally defined by Range Safety at the launch facility, the primary payload of the particular launch or the CubeSat integrator may have a requirement that might limit 


\section{CUBESAT MISSION PLANNING TOOLBOX}

transmitter power. The MAP may also be limited by the radio frequency ampilifer (RF AMP) or transmitter used. The MAP is defined as power at the transmitters antenna input. The MAP is measured in watts.

\section{Amplifier Efficiency}

Amplifier Efficiency is how efficient the amplifier is during the simulation. Typically a RF amplifier will be around 50-60 \% efficient. Iif the efficiency is $50 \%$, then for every watt that the amplifier needs to output, two watts need to be supplied to the amplifier. This parameters will help determine the power and current densities used during communication.

\section{Downlink Data Rate}

CMPT allows a user to select popular data rate options shown in the list below. CMPT assumes a single pulse equals a single bit. This assumption simplies calculations.

Downlink Data Rate Options

- 1200 bps

- 2400 bps

- 9600 bps

- 19600 bps

\section{Size of Packet Overhead}

In packet radio transmission such as those used in CubeSat communications, traffic is put into packets. For most CubeSat communications, AX.25 packets are used to packetize the data. A AX.25 packet can be as large as 
30 bytes [2]. This input will be used when calculating the percent of data that was downlinked.

\section{Duty Cycle}

One of the major issues with any transmitter and more specifically the RF AMP used for transmission is the heat generated from continuous transmission. One of the ways to circumvent over heating is to transmit periodically over the course of the transmission. That ratio is the duty cycle and it is integral in determining the time it takes to downlink data as well as the power density equations, both are described in section 4.4.

\section{Spacecraft Antenna Gain}

Most CubeSat flown in the past have used a dipole antenna with about 5 dB of gain [38]. Higher frequency systems, such as those systems that use S band or the $900 \mathrm{MHz}$ Industrial, Scientific and Medical (ISM) band, will use either custom monopole or patch antennas such as Claude Space's S Band Patch Antenna with 8 dB gain. [39]

\section{Ground Station Antenna Gain}

The Ground Station Antenna Gain should be much larger than the spacecraft's antenna gain, because the use of larger fixed antenna can increase the gain. For $420 \mathrm{MHz}$ Yagi antenna a typical gain is 10 to $16 \mathrm{~dB}$ [21] and the antenna gain for higher frequencies is typically around $21 \mathrm{~dB}$ [40]. 


\section{CUBESAT MISSION PLANNING TOOLBOX}

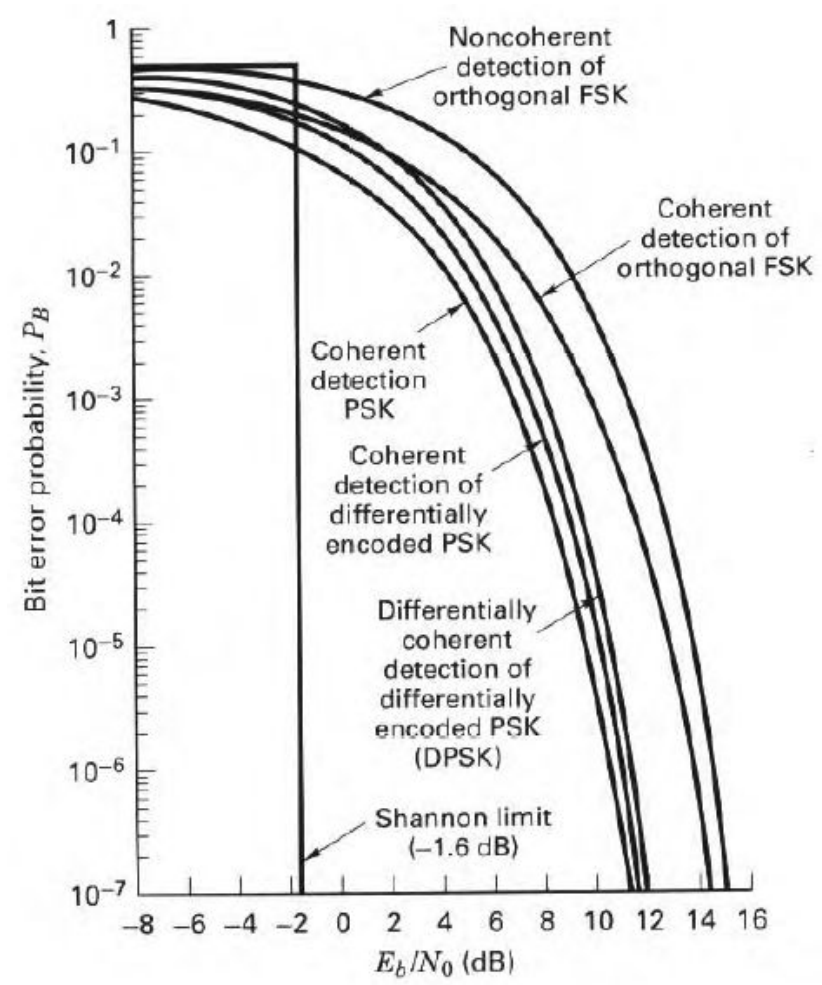

Figure 4.11: BER vs. $E_{b} / N_{0}$ chart showing the required $E_{b} / N_{0}$ in dBs for different modulation schemes. Refer to the Noncoherent detection of orthogonal FSK for desired $E_{b} / N_{0}$ [41].

\section{Desired $E_{b} / N_{0}$}

The bit error rate (BER) is the principal performance metric that is a measure of quantization error in digital communications links. It is possible to predict the BER of a communication link as a function of the bit-energy to noise-spectral-density ratio, denoted $E_{b} / N_{0}[3]$.

\section{Noise Temperature}

Noise Temperature is a complex parameter to establish for satellite developers. The equations below are component dependent and can vary dramatically over the course of an orbit. The noise temperature is the thermal noise in the resistance of the signal source that is the fundamental 
limit of achievable signal sensitivity.

$$
T_{S}=T_{A} * L+(1-L) T_{L}+(F-1) * T_{0}
$$

$T_{A}$ is antenna noise temperature, $T_{L}$ is the effective temperature of the feed and other passive components, L is the loss through the antenna feed and passive components upstream of the receiver, $\mathrm{F}$ is the receiver noise figure.[3]

$$
N_{0}=k * T_{S}
$$

$N_{0}=$ noise power spectral density, $T_{S}$ is the effective thermodynamic temperature of the modeled resistance. $\mathrm{K}$ is the Boltzmann constant $=-228.6 d B J / K[3]$. The $N_{0}$ will be used in the power required calculations in section 4.4.6.

\section{Line Losses}

Line loss is signal degradation internal to the satellite that is associated with cabling and connectors that might be present in the communication link. Most of these losses are negligible in a CubeSat because of short cable lengths and a small number of connectors. However, if there are noise-generating components around the cable's route or there are many connectors in the cable route, the losses could become an issue. For most cases a loss of $1 \mathrm{~dB}$ is sufficient and could be described an extreme worst case for most missions. 


\section{CUBESAT MISSION PLANNING TOOLBOX}

\section{Pointing Losses}

The pointing loss parameter is antenna specific and can be determined during the design phase of your communication system. Typically for a dipole that has an omni-directional radiation pattern the pointing loss is small, with a typical pointing loss from $.3 \mathrm{~dB}$ to $8 \mathrm{~dB}$. [42] The large range of values is reuslting from the use of different kinds of antennas. An omnidirectional antenna will have low pointing losses while a helix or parabolic dish will have dramatically higher pointing losses due to the directionality of the antenna.

\section{Downlink Losses}

Downlink losses is signal degradation associated with the attenuation along the downlink path such as weather or atmospheric gases. The downlinking losses are approximately $2 \mathrm{~dB}$ for $430 \mathrm{MHz}$ [3], but can be larger if it is raining around the ground station receiver.

\section{Margin}

Margin is a buffer between what you expect and the worst case scenario in your communication link. Depending on the level of complexity within a system, the risk associated with the system will affect the margin needed. In the initial stages of a design, the margin should be large and decrease as development matures. 


\subsection{CMPT Summary}

The summary dialog window as shown in figure 4.12 is displaying the calculations and displays from running the simulation.

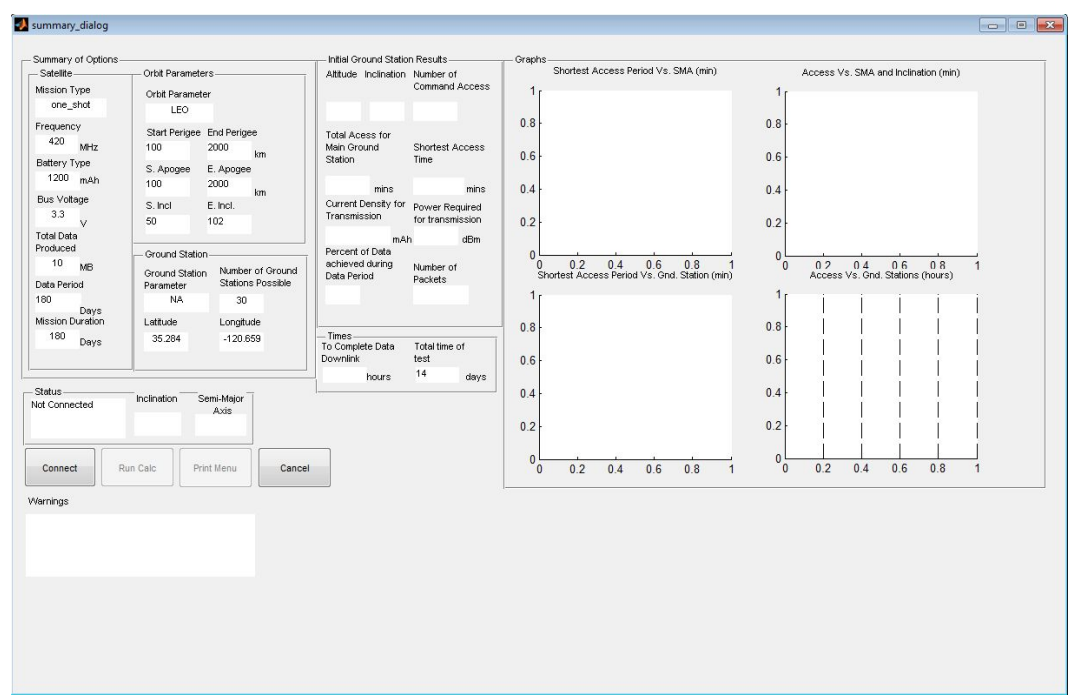

Figure 4.12: Screenshot of the CMPT Summary Window

\subsubsection{Summary of Options Panel}

The Summary of Options panel shown in figure 4.13 and gives a simplified reminder of the major options selected during setup.

\begin{tabular}{|c|c|c|}
\hline \multicolumn{3}{|c|}{ —Satellite } \\
\hline $\begin{array}{l}\text { Mission Type } \\
\text { custom }\end{array}$ & $\begin{array}{l}\text { Orbit Parameter } \\
\text { custom }\end{array}$ & \\
\hline Frequency & Start Perigee & End Perigee \\
\hline $420 \quad \mathrm{MHz}$ & 200 & 800 \\
\hline $\begin{array}{l}\text { Battery Type } \\
4400 \text { mah }\end{array}$ & S. Apogee & E. Apogee \\
\hline Bus Vottage & S. Incl & E. Ind. \\
\hline${ }_{\text {Total Data }}^{5} \vee$ & 50 & 98 \\
\hline Produced & - Ground Station & \\
\hline $\begin{array}{l}\text { B000 MB } \\
\text { Data Period }\end{array}$ & $\begin{array}{l}\text { Ground Station } \\
\text { Parameter }\end{array}$ & $\begin{array}{l}\text { Number of Ground } \\
\text { Stations Possible }\end{array}$ \\
\hline 5 & NA. & 30 \\
\hline Mission Duration & Lattude & Longitude \\
\hline 365 Days & 35.284 & -120.659 \\
\hline
\end{tabular}

Figure 4.13: Screenshot of the Summary of Options Panel 


\section{CUBESAT MISSION PLANNING TOOLBOX}

\section{Satellite}

The Satellite Panel displays the main parameters of the mission and satellite.

\begin{tabular}{|l|c|}
\hline Parameter & Reference \\
\hline mission type & section 4.1.1 \\
frequency & section 4.1.5 \\
battery type & section 4.1.5 \\
bus voltage & section 4.1.5 \\
total data produced & section 4.1.6 \\
data period & section 4.1.6 \\
mission duration & section 4.1.5 \\
\hline
\end{tabular}

Table 4.3: Parameters and references for each of the Satellite Parameters

\section{Orbit Parameters}

The Orbits Parameters panel displays the type of orbit selection; start and end perigee; start and end apogee; and start and end inclination. Section 4.1.4 gives more detail about the orbital parameters.

\section{Ground Station}

The Ground Station panel displays the ground station selection, number of possible ground stations, latitude and longitude for the main station. Section 4.1.3 gives more detail about the ground station parameters. 


\subsubsection{Times Panel}

The times panel displays the times calculated in the initial stages of the simulation. In the times panel, the complete data for downlink, and total time of test.

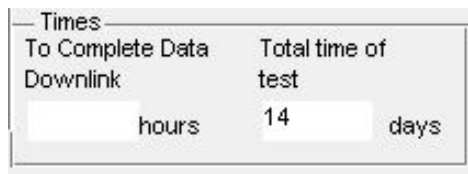

Figure 4.14: Screenshot of the Times Panel

\section{Complete Data Downlink Time}

The complete data downlink input dispalys the amount of time to downlink the experimental date. Equation 4.4.2.1 calculates the complete data downlink. Right clicking on the text box will rotate the units from minutes to days and hours.

\section{Total Time of Test}

The total time of test displays the time of the simulation. If the data period (section 4.1.6) is less than the test time (section 4.1.2) then the time of the simulation will change to the data period. The decreased time period will give a better idea of the data period. 


\subsubsection{Initial Ground Station Results}

During the simulation, the access reports for the main ground station are processed where the total and longest access times are calculated. Then the orbit with the greatest contact period will be set as the main orbit for the rest of the simulations.

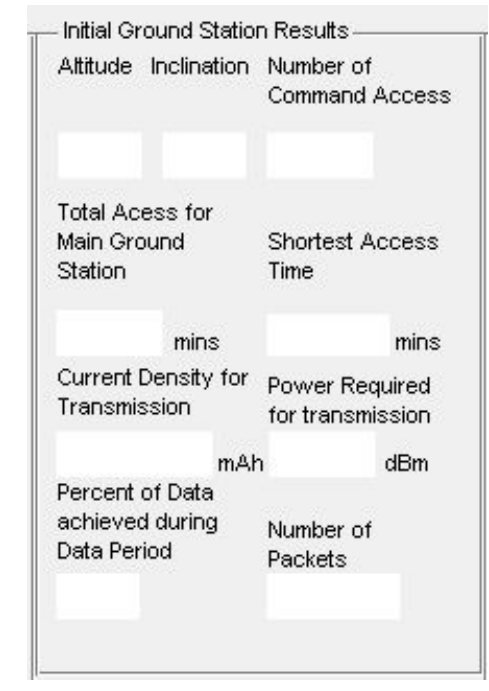

Figure 4.15: Screenshot of the Initial Ground Station Results Panel

\section{Optimal Altitude and Inclination}

The optimal orbit that has the greatest amount of $T_{\text {optimal }}$ as shown in the equation 4.2.3.1; $T_{\text {accessalt,inc }}$ is the longest access period for each altitude and inclination and $N_{\text {accessalt,inc }}$ is the number of accesses in each of the altitudes and inclinations under test.

$$
T_{\text {optimal }}=\max \left(T_{\text {accessalt }, \text { inc }} * N_{\text {accessalt }, \text { inc }}\right)
$$




\section{Number of Command Accesses}

The number of command accesses text box displays how many opportunties a satellite operator will have to access their satellite. A command access is any access period over the main ground station longer than seven minutes.

\section{Total Access for Main Ground Station}

The total access for main ground station text box displays the total amount of time a user will have to downlink data. Total access is measured in minutes. Total access is also calculated using direct line of sight with the satellite, which could be improved in future revisions of CMPT to allow for more ground station customization.

\section{Longest Access Time}

The longest access time displays the longest acess for the desired orbit.

\section{Current Density for Transmission}

The current density for transmission text box displays the amount of mAh will be used when transmitting during the time of test. This allows for a user to understand how much of the battery they will be using during testing.

\section{Power Required for Transmission}

The power required for transmission text box displays the power required to complete the communication link in $\mathrm{dBm}$. The equations are described in section 4.4 .5 


\section{CUBESAT MISSION PLANNING TOOLBOX}

\section{Percent of Data achieved during Data Period}

The percent of data achieved during Data Period text box displays how much of the experimental data the main ground station downlinked.

\section{Number of Packets}

The number of packets text box displays the maximum number of packets recommend for downlinking the experimental data.

\subsubsection{Graphs Panel}

The graphs populate as the simulation runs. The MATLAB Graphical User Interface (GUI) limits the abilities to use axis labels. There are no axis labels and the units are shown in the title of each graph.

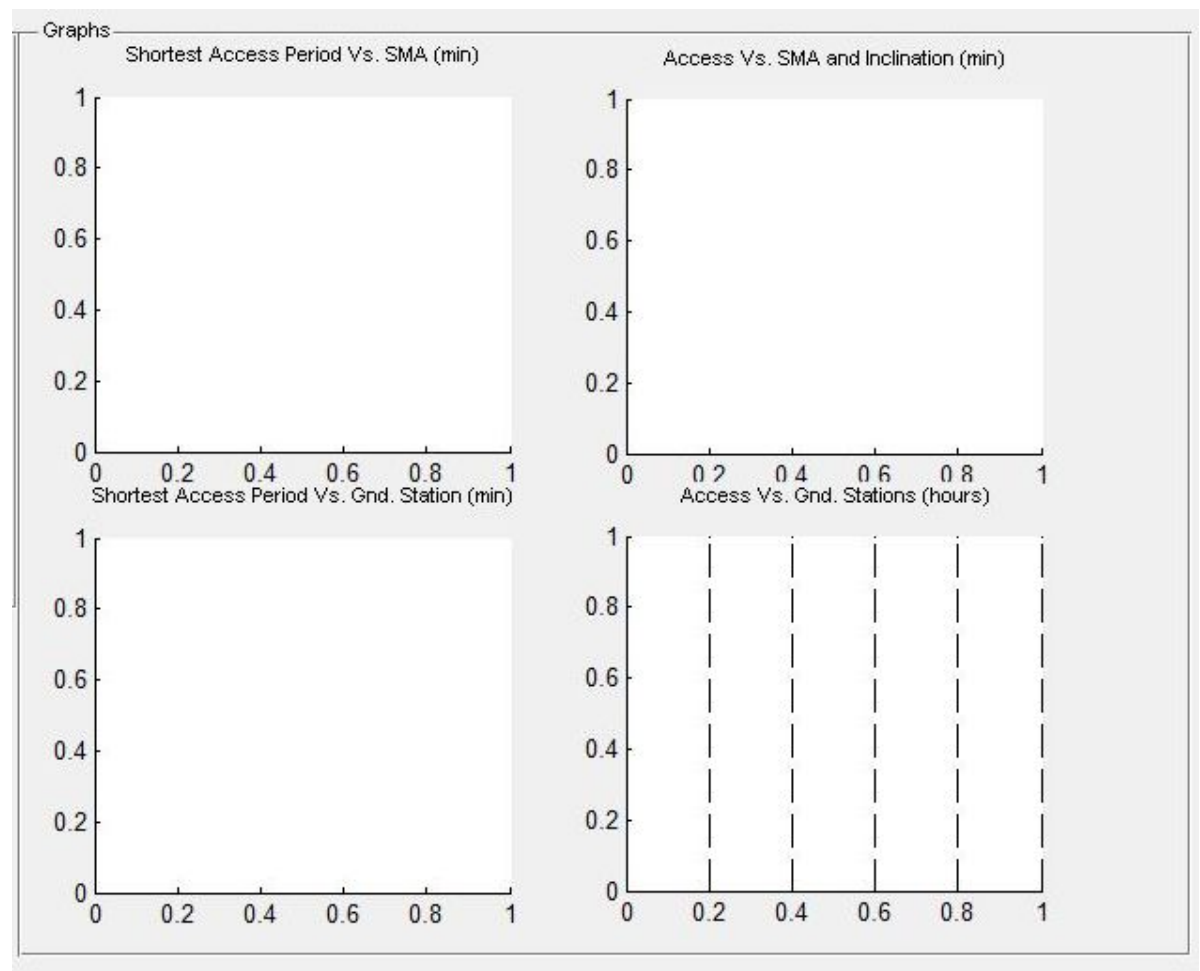

Figure 4.16: Screenshot of the Graphs Panel 


\subsection{CMPT Summary}

\begin{tabular}{|l|c|c|c|}
\hline Title & $\mathrm{X}$ & $\mathrm{Y}$ & $\mathrm{Z}$ \\
\hline Longest Access vs. Semi Major Axis and Inclination & $\mathrm{km}$ & degrees & minutes \\
Access vs. Semi Major Axis and Inclination & $\mathrm{km}$ & degrees & minutes \\
Longest Access vs. Number of Ground Stations & Number of Ground Stations & minutes & N/A \\
Access Vs. Number of Ground Stations & Number of Ground Stations & hours & N/A \\
\hline
\end{tabular}

Table 4.4: Title and axis of the Graphs Panel

\subsubsection{Specifics Panel}

After the simulations run, CMPT allows users to examine many of the important characteristics of the simulations. A user can select any semi major axis, inclination, and number of ground station option. The outputs are shown in table 4.5

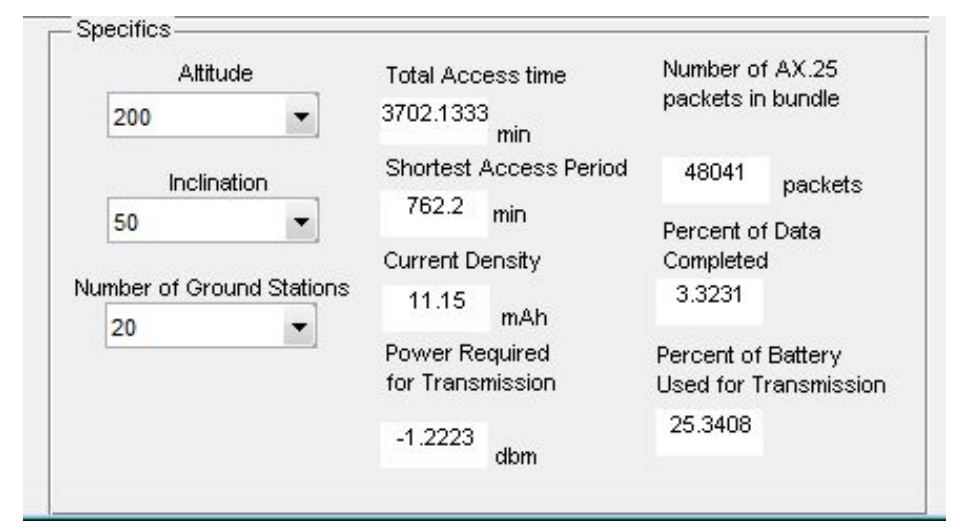

Figure 4.17: Screenshot of the Specifics Panel

\begin{tabular}{|c|c|c|}
\hline Output & Description & Reference \\
\hline total access times & $\begin{array}{l}\text { Shows the Total Access time and allows the user to change } \\
\text { the units of the output by right clicking the value changing } \\
\text { total access to days, hours, or minutes. }\end{array}$ & section 4.4 .3 \\
\hline longest access period & $\begin{array}{l}\text { Shows the longest access of the desired semi major axis, in- } \\
\text { clination and ground station. }\end{array}$ & section 4.4 .4 \\
\hline current density & Shows the amount of battery that used during transmission. & section 4.4 .7 \\
\hline power required for transmission & $\begin{array}{l}\text { Shows the power in } \mathrm{dBm} \text { that will be required out of the } \\
\text { satellite transmitter. }\end{array}$ & section 4.4 .6 \\
\hline number of AX.25 packets in & Shows the number of packets downlink during one cycle. & section 4.4 .11 \\
\hline percent of data completed & $\begin{array}{l}\text { Displays the percent of data downlinked during the simula- } \\
\text { tion. }\end{array}$ & section 4.4 .12 \\
\hline percent of battery used for tra & Shows the percent of battery used while downlinking data & \\
\hline
\end{tabular}

Table 4.5: The Specifics Panel Outputs and their Reference 


\section{CUBESAT MISSION PLANNING TOOLBOX}

\subsubsection{Status and Warnings}

The status and warnings section of the Summary window give the user an idea of where CMPT is at in the simulation. This section gives control to the user. Finally, this section informs the user if anything changes in the paramters used.

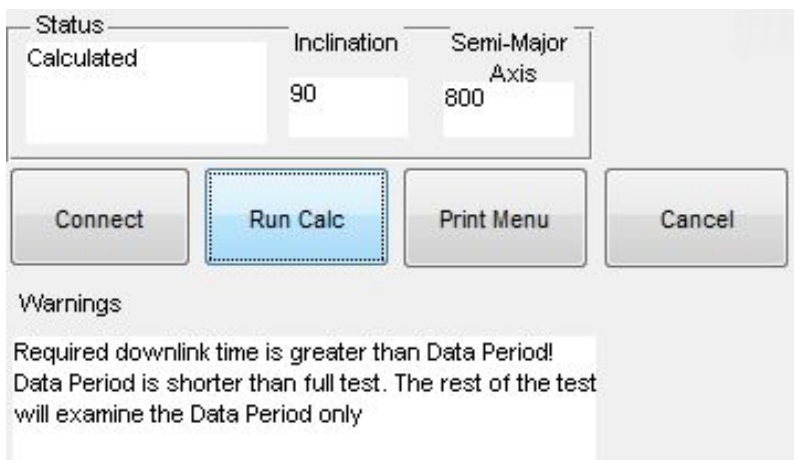

Figure 4.18: Screenshot of the Status and Warnings Panels

\begin{tabular}{|c|c|}
\hline Component & Description \\
\hline \multicolumn{2}{|l|}{ Status Panel } \\
\hline status textbox & Lets the user know what stage of the simulation CMPT is at. \\
\hline inclination and semi-major axis & Shows the current Inclination and Semi-Major Axis under test \\
\hline \multicolumn{2}{|l|}{ Buttons } \\
\hline Connect & Initiates the connection with STK and loads the scenario. \\
\hline Run Calc & Runs the Simulations and calculates the outputs. \\
\hline Print Menu & Brings up the Print and Results window. \\
\hline Cancel & Exits and clear memory. \\
\hline \multicolumn{2}{|l|}{ Warnings } \\
\hline warnings textbox & $\begin{array}{l}\text { Shows a message if any of the user's parameters have changed be- } \\
\text { cause of power restrictions or time of test adjustments. }\end{array}$ \\
\hline
\end{tabular}

Table 4.6: The Status and Warnings Section Summary 


\subsection{CMPT Printing and Suggestions}

CMPT gives the user suggestions based on the calculations performed during the simulation. CMPT also allows a user to print graphs and tables of important parameters.

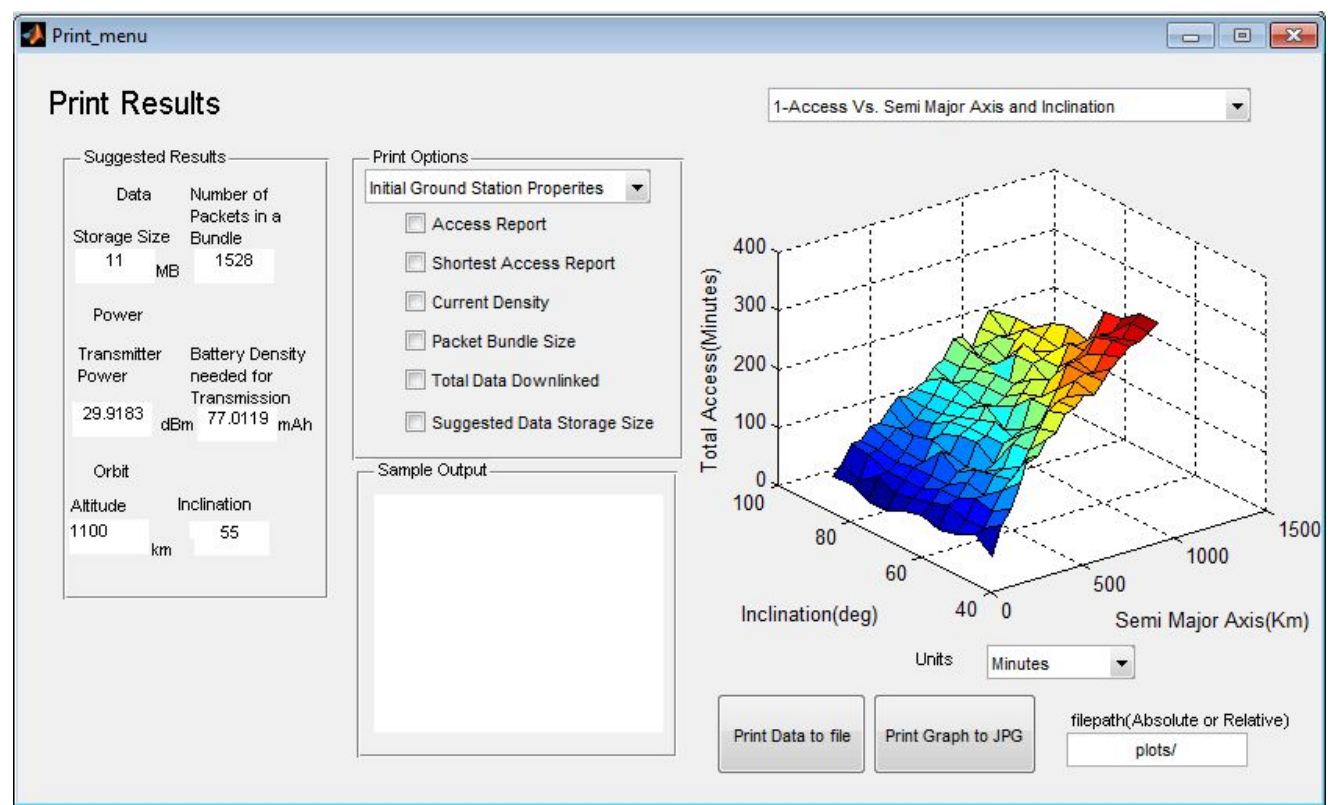

Figure 4.19: The print results window of CMPT provides suggested parameters and print options. 


\section{CUBESAT MISSION PLANNING TOOLBOX}

\subsubsection{Suggested Results}

After execution of the simulation, CMPT calculates suggested values for the user's CubeSat mission. Section 4.4 shows the calculations for these values. The Suggested Results section shows values such as storage size, power out of transmitter and other parameters.

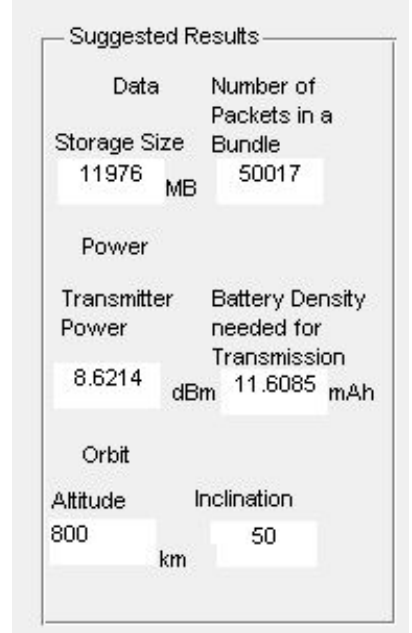

Figure 4.20: Screenshot of the Suggestion section in the Print Menu Window 


\subsubsection{Print Options and Sample Output}

This section allows a user to print the data into a tabular form such as seen in figure 4.21a. At the time of writing, units are fixed but may be adjustable in a future release of CMPT. In clockwise order, starting from top left, the elements are: total access, longest access, current density used for transmission, number of packets used,total data downlinked, and storage size.

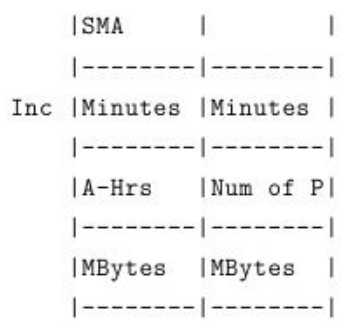

(a) A sample

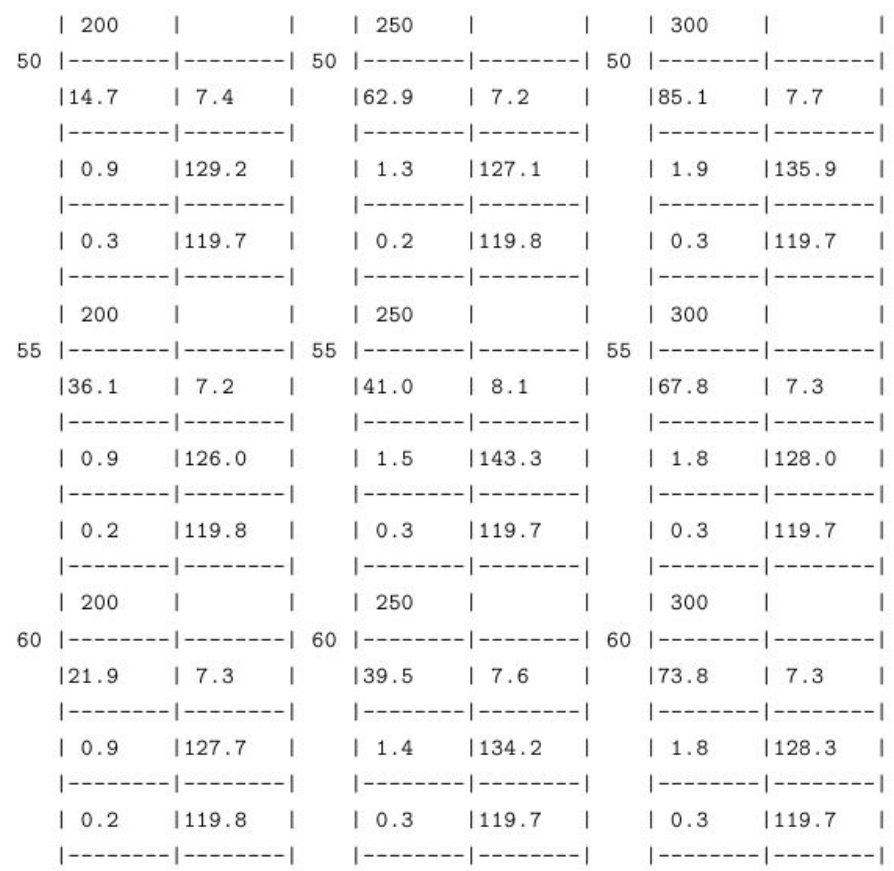

(b) The output

Figure 4.21: Output of CMPT print in tabular Form. 


\section{CUBESAT MISSION PLANNING TOOLBOX}

\subsubsection{Graphs}

CMPT enables the user to print the graphs and easily use them in another program, including total access, longest access, power densities, transmission requirements, and packet bundle size. CMPT generates a .jpg file in the folder shown in the filename box.

Note: the folder destination needs to exist before saving the file. Units are selectable in the pull down menu below the graphs.

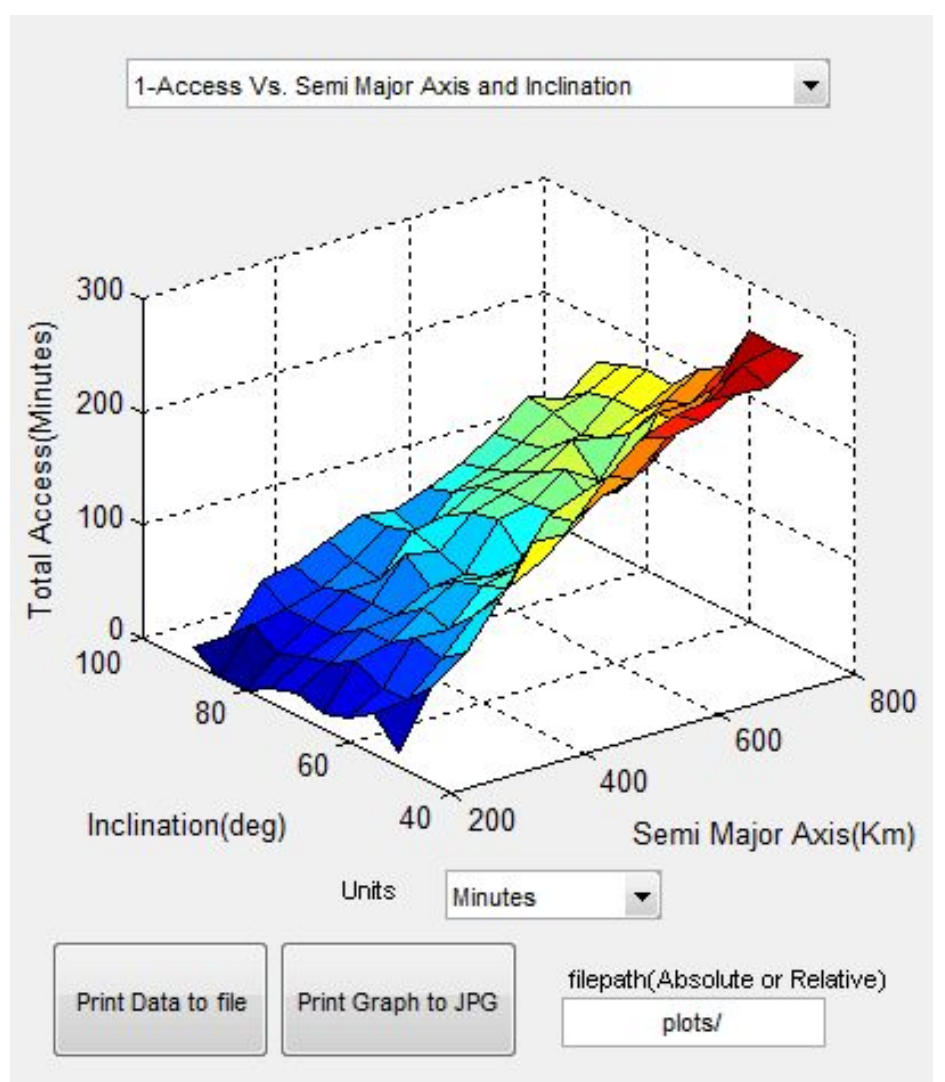

Figure 4.22: Screenshot of the Access vs. SMA vs. Inc in minutes 


\subsection{Calculations}

\subsubsection{Time Calculations Introduction}

In the time calculation section, all the equations associated with downlinking time and access times are explained.

\subsubsection{Downlink Time}

It is important to know that total amount of time a collection of data will take a few reasons. The downlink time will give you an idea of how realistic the experimental will be to downlink. This can assist in figuring how often to generate your data too. The downlink time also helps with the percent downlinked calculation later section 4.4.12.

$$
T_{\text {downlink }}=D_{\text {produced }} /\left(D_{\text {rate }} * C\right)
$$

$D_{R}=$ data rate in bps, $T=$ access time, $C=$ duty cycle, and $T_{D}=$ Downlink Time.

\subsubsection{Total Access Time}

The total access time is calculated using MATLAB that can be seen in the code section of the appendix D.

\subsection{4 longest Access Period}

The longest access period is found by looking at all the access times after calculating the total access time. Reference the important code section of the appendix D for more information. 


\section{CUBESAT MISSION PLANNING TOOLBOX}

\subsubsection{Electrical Calculations Introduction}

Some of the most useful calculations provided by CMPT are explained in this section. These equations include the calculations for the power required for downlinking, power density and current density equations.

\subsubsection{Power Required for Downlinking}

The power required for transmitting parameter is used to develop link budgets. Satellite developers use a link budget that takes values such as data rate(sec 4.1.7), altitudes, line losses(sec 4.1.7) and other parameters to engineer their communication link. One of the major contributions of completing a link budget figuring out if the communication link will close.[3] A closed link budget means that the satellite will be able to communicate effectively with the ground station and with enough margin(in the form of extra $\mathrm{dBs}$ ) to make sure that the system will work if the system experiences undesirable weather or part of the satellite fails to function properly.

$$
S_{R}=R_{e} *\left(\sqrt{\frac{R_{m o}^{2}}{R_{e}^{2}}}-\cos \left(\frac{A_{e} * \pi}{180}\right)^{2}-\sin \left(\frac{A_{e} * \pi}{180}\right)\right)
$$

$R_{e}=$ radius of earth, $R_{m o}=$ median radius of orbit, $A_{e}$ is the effective area,and $S_{r}=$ slant range. The slant range is used in the path loss equation. $[43]$

$$
L_{p}=22+20 * \log 10\left(\frac{S_{R} * 1000}{\lambda}\right)
$$

$L_{p}$ is the path loss indBs, and $\lambda$ is the wavelength in meters. Path loss is used in the link budget and is the major contributor to signal degradation 


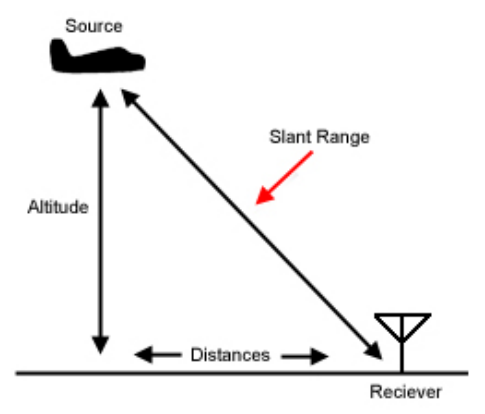

Figure 4.23: Visualization of Slant with an Airplane Sourced from the Boston Over Flight Noise Study

in the communication link. [43]

$$
D_{r d B H z}=10 * \log 10\left(D_{r}\right)
$$

$D_{r}$ is the data rate in bits per second. $D_{r_{d B H z}}$ is measured in dBs with a reference to one hertz

$$
E_{b} / N_{0_{s y s}}=M+E_{b} / N_{0_{r e q}}
$$

$M$ is link margin, which is defined in the initial planning stages as essentially the buffer a user has between worst case and the working case. $E_{b} / N_{0_{s y s}}$ is the Energy per bit to noise power density ratio. It is equivalent to the signal to noise ratio and is the parameter of choise for digital links. [43]. The difference between the $E_{b} / N_{0_{r e q}}$ and $E_{b} / N_{0_{s y s}}$ is that the $E_{b} / N_{0_{r e q}}$ is what is needed to maintain quality communications.

$$
S / N_{0}=E_{b} / N_{0_{s y s}}+D_{r d B}
$$




\section{CUBESAT MISSION PLANNING TOOLBOX}

$S / N_{0}$ is the signal to noise ratio and is measured in $\mathrm{dBHz}$.

$$
F o M=G_{R}-L_{l}+10 * \log 10\left(N_{T}\right)
$$

$G_{R}$ is the gain of the receiver antenna(section 4.1.7) on the ground, $L_{l}$ is the line losses (section 4.1.7) associated with the ground link, and $N_{T}$ is the noise temperature of the receiver (section 4.1.7). The FoM is the ultimate measure of the receiver's performance [43].

$$
\text { IsoSignalLevel }=S / N_{0}-L_{\text {pointing }}+C_{b}+F o M
$$

IsoSignalLevel is the signal to noise power density. $L_{\text {pointing }}$ is the loss associated with antenna pointing.

$$
S C E I R P=I \text { soSignalLevel }+L_{p}
$$

SCEIRP is the isotropic signal level at the ground station [3].

$$
P_{d B W}=S C E I R P-L_{l}-G_{T}
$$

$P_{d B W}$ is the power out of the transmitter on the satellite in dBW.

$$
P_{d B m}=P_{d B W}+30
$$

$P_{d B m}$ is the power out of the transmitter on the satellite in $\mathrm{dBm}$.

$$
P_{W}=10^{\frac{P_{d B}}{10}}
$$

$P_{W}$ is the power out of the transmitter on the satellite in watts. 


\subsubsection{Current Density}

A large portion of the power budget is dedicated to the communication system. Transmitters typically require a significant amount of the CubeSat's overall power, but historically, this category has been over-budgeted. To assist future CubeSat developers in figuring out the correct numbers to put in their power budget and ultimately build a more efficient satellite, the program will calculate the power density in Watt-hours which will contribute to the Current Density equation 4.4.7.2 givies an approximate measure of the amount of battery that will be used during transmission.

$$
D_{\text {power }}=C * t_{\text {short }} * P_{W} * \frac{1}{e f f}
$$

$D_{\text {power }}$ is the Power density used during transmission. $C$ is the duty cycle(section 4.1.7) of the transmitter. $t_{\text {short }}$ is the time of the longest access period during the simulation. $P_{W}$ is the power needed to complete the link found in equation 4.4.6.11. ef $f$ is the efficiency of the transmitter.

$$
D_{\text {current }}=\frac{D_{\text {power }}}{V_{\text {bus }}}
$$

$D_{\text {current }}$ is the Current Density which will be used in equation 4.4.8.1. $V_{\text {bus }}$ is the bus voltage as described in section 4.1.5

\subsubsection{Percent of Battery Used for Transmission}

$$
P_{\text {battery }}=\frac{D_{\text {current }}}{D_{\text {batt.total }}} * 100
$$

The percent of the battery used for transmission is calculated using the equation 4.4.8.1. Battery discharge is not taken into account for this cal- 
culation, however in future versions when the power subsystem is modeled as well, battey discharge will be addressed.

\subsubsection{Data Calculations Introduction}

In the early stages of development of any CubeSat, data requirements provide the computer engineer with lots of information to consider when designing spacecraft components. Once the data requirements are proposed, they need to be verified. To do this a CubeSat Developer might assume a generic orbit and try to estimate an acceptable data range. CMPT improves these estimations by providing more accurate values, such as access time, which will allow for developers to plan their mission more effectively.

\subsubsection{Total Packet Bundle Size}

CMPT uses the longest access times of each option: altitude, inclination and the amount of ground station that will create the best-case packet size for the transmission capability of the system.

$$
D_{\text {total }}=T_{\text {access }} * D_{\text {rate }} * C * N_{\text {accesses }}
$$

$D_{\text {total }}$ is total amount of bits that can be downlinked. $N_{\text {accesses }}$ is the number of accesses during the time of test. This value will be used in equation 4.4.11.1.

\subsubsection{Number of Packets}

The AX.25 protocol defines packets at a maximum of 256 bytes [2]. This equation uses the maximum size of a packet for the calculation. After the 
number of packets that can be transmitted are calculated the amount of actual data that is downlinked is calculated and

$$
N_{\text {packets }}=D_{\text {total }} /(256 * 8)
$$

$N_{\text {packets }}$ is the number of packets that can be downlinked during the test.

\subsubsection{Percentage of Data Downlinked}

The percent of data is data downlinked divided by data produced during the data period. During the initial design phases of a CubeSat this number will be helpful in determining the feasiblity of a mission. This could be helpful in determining the period between experiments, or if the data generation parameters needs to be changed.

$$
D_{\text {percent }}=\frac{N_{\text {packets }} *\left(256-D_{\text {overhead }}\right) * 8}{D_{\text {produced }}}
$$

$D_{\text {produced }}$ is the total amount of data generated in bits during the experiment. $D_{\text {overhead }}$ is the total amount of overhead in the packet header in bytes. $D_{\text {percent }}$ is the percent of data that was downlinked during the test.

\subsubsection{Suggested Data Storage Size}

The suggested Data Storage Size estimates the minimum amount of storage a user would want on their satellite.

$$
D_{\text {storage }}=D_{\text {total }} *\left(2-D_{\text {percent }}\right)
$$


$D_{\text {storage }}$ is the minimum size of the data storage required for a mission and is measured in bits. 


\section{5}

\section{Case Studies}

This chapter examines how CMPT can be applied to different mission types. Initially, there will be an assessment of the base cases and comparing the parameters presented for each case. Then real world missions will be input into CMPT. All of these test cases will be using the custom option (sec 3.4.5), since they are established which enables all of their parameters to be put into the CMPT. In most cases there will be a comparison between the BC method and the GSI downlink method. The two science mission case studies are a comparsion of an older mission and a more recent mission using a full world network and more data production. When values were not found through published papers, or other resources, the values from a well-established project, CP5, are used. 


\section{CASE STUDIES}

\subsection{Base Cases}

This section will examine the built-in cases for each mission type. In each of the sections, the print menu from CMPT will be shown and at the end of this section there will be a summary of the suggestions for each case.

\subsubsection{Initial Attempt}

This mission was run in CMPT with LEO orbital parameters and a North American ground station network, CMPT recommends a bundle size of 316 packets and a transmit power of $29.9 \mathrm{dBm}$. CMPT also suggests a minimum storage size of $14 \mathrm{MB}$.

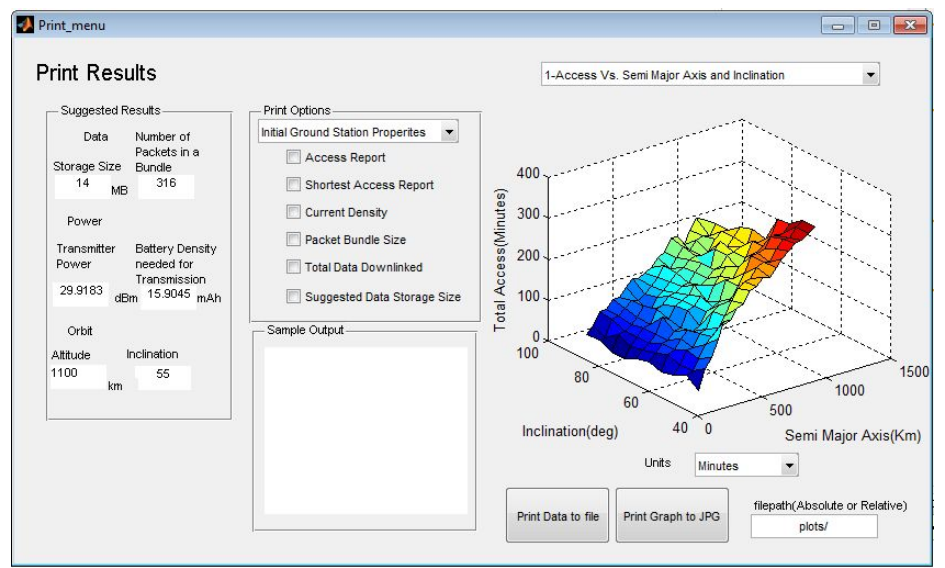

Figure 5.1: Screenshot of print menu for the Initial Attempt mission type 


\subsubsection{One-Shot}

This mission was run in CMPT with LEO orbital parameters and a Full World ground station network, CMPT recommends a bundle size of 255 packets and a transmit power of $29.9 \mathrm{dBm}$. CMPT also suggests a minimum storage size of $10 \mathrm{MB}$. The power requirements for both the Initial and One-Shot are the same because the orbit optimization is based on the most access time over the main ground station, and the transmitter properties.

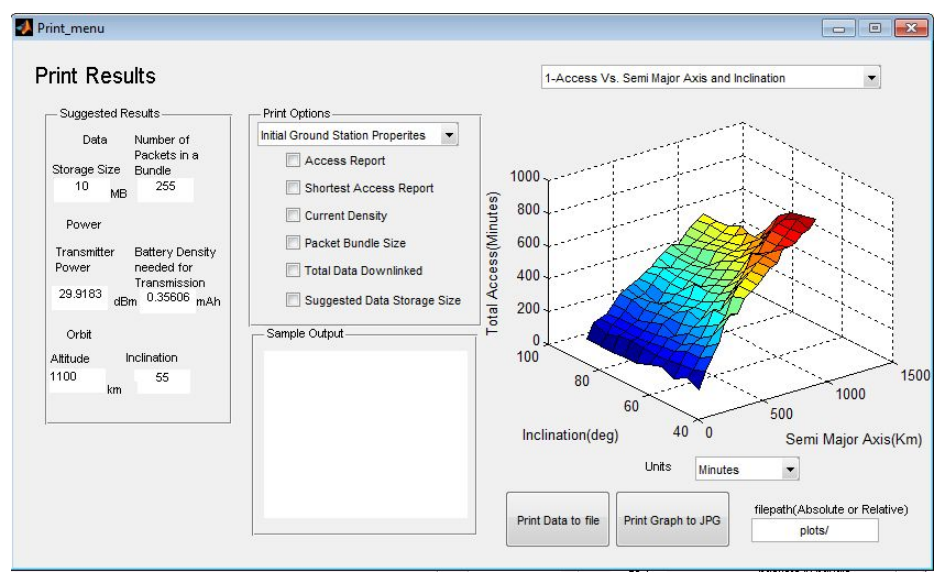

Figure 5.2: Screenshot of Print Menu for the One-Shot mission type 


\section{CASE STUDIES}

\subsubsection{Science Mission}

This mission was run in CMPT with LEO orbital parameters and a Full World ground station network, CMPT recommends a bundle size of 4,125 packets and a transmit power of $23.3 \mathrm{dBm}$. The program also suggests a minimum storage size of $161 \mathrm{MB}$.

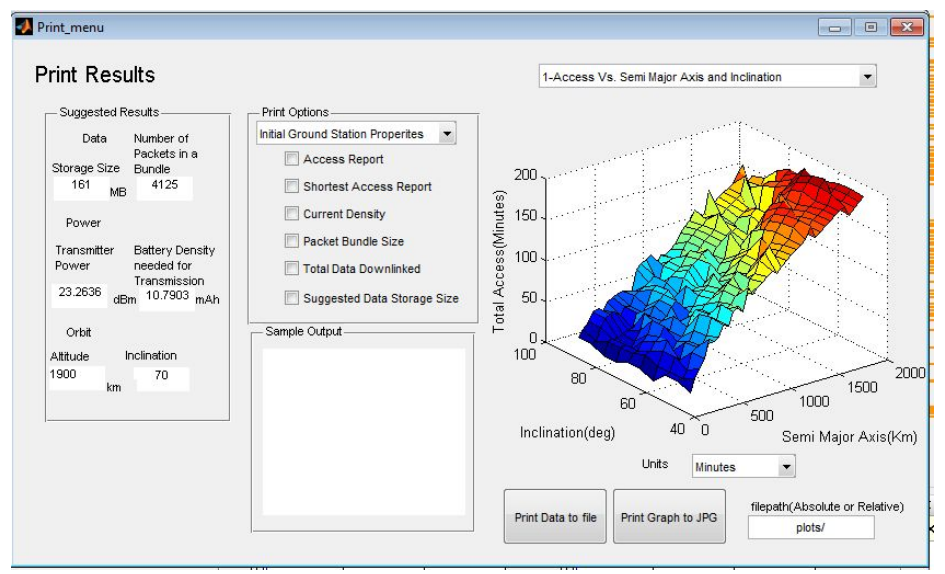

Figure 5.3: Screenshot of Print Menu for the Science mission type 


\subsubsection{Industry Test Bed}

This mission was run in CMPT with LEO orbital parameters and a North American ground station network, CMPT recommends a bundle size of 3,636 packets and a transmit power of $29.7 \mathrm{dBm}$. The program also suggests a minimum storage size of $147 \mathrm{MB}$.

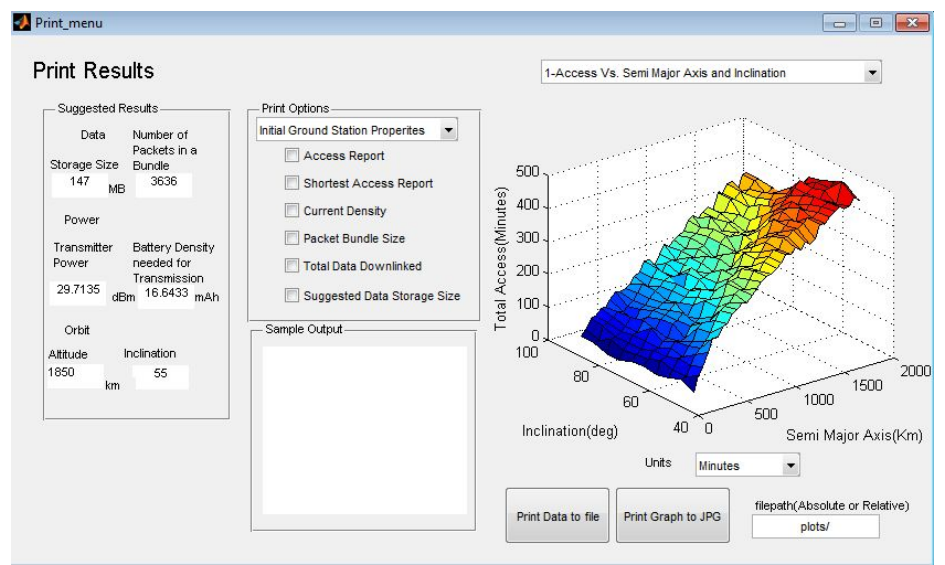

Figure 5.4: Screenshot of Print Menu for the Industrial Test-Bed Mission Type 


\subsubsection{Summary of Suggestions}

The table 5.1 shows the results for each of the mission type.

\begin{tabular}{|l|l|l|l|l|c|c|}
\hline Mission Type & Storage Size & Bundle Size & Transmit Power & Battery Density & Optimal Altitude & Optimal Inclination \\
\hline Initial Attempt & $14 \mathrm{MB}$ & 316 Packets & $29.9 \mathrm{dBm}$ & $15.9 \mathrm{mAh}$ & $1100 \mathrm{Km}$ & $55 \mathrm{deg}$ \\
\hline One-Shot & $10 \mathrm{MB}$ & 255 Packets & $29.9 \mathrm{dBm}$ & $.35 \mathrm{mAh}$ & $1100 \mathrm{Km}$ & $55 \mathrm{deg}$ \\
\hline Science & $161 \mathrm{MB}$ & 4,125 Packets & $23.26 \mathrm{dBm}$ & $10.79 \mathrm{mAh}$ & $1900 \mathrm{Km}$ & $70 \mathrm{deg}$ \\
\hline Test Bed & $147 \mathrm{MB}$ & 3,636 Packets & $29.7 \mathrm{dBm}$ & $16.64 \mathrm{mAh}$ & $1900 \mathrm{Km}$ & $70 \mathrm{deg}$ \\
\hline
\end{tabular}

Table 5.1: Summary of Suggests for each base case.

In the table 5.1 there are some differences between the types of missions, to include lower transmit power for the science(sec 3.4.3) and test bed (sec 3.4.4) missions. The lower transmit power could be attributed to the higher gain antenna on the ground for each of these missions. Another advantage the science ( $\sec 3.4 .3)$ missions and test bed missions have over the others missions is the higher optimal orbit allowing for longer access times over the ground stations. 


\subsection{Real World Cases}

This section examines past and present missions to see how CMPT performs. This section will examine possible improvements to this missions. Finally this section provide a comparsion between the GSI (sec 2.2.6) downlink method and the BC (sec 2.2.6) downlink method. The CubeSat missions that are going to be analyzed are: CP6 for the Initial Attempt case, CP5 for the One-Shot case, QuakeSat for the science mission with a single ground station, and RAX-2 for the science case with a full world ground station network.

\begin{tabular}{|l|c|c|c|c|}
\hline Mission & Type & Orbit & Ground Station Option & Mission Length \\
\hline CP6 & Initial Attempt & LEO & Full World & 180 days \\
CP5 & One-Shot & LEO & Full World & 90 days \\
QuakeSat & Science & LEO & Single Station & 180 days \\
RAX-2 & Science & LEO & Full World & 365 days \\
\hline
\end{tabular}

Table 5.2: Summary of Real World Cases 


\section{CASE STUDIES}

\subsubsection{Initial Attempt: CP6}

The primary mission of CP6 was to im-

plement an attitude control system using

only magnetic torquers embedded within the side panels. Attitude determination is performed using two-axis magnetometers on each side panel. Once the primary objectives had been met, a command will

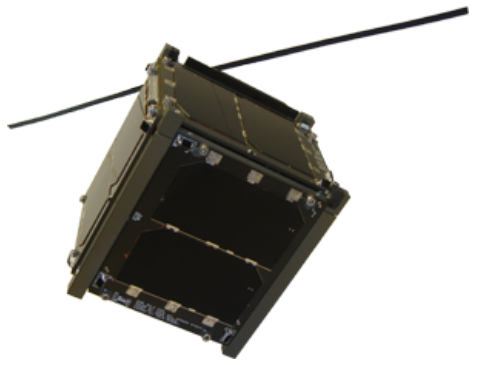

Figure 5.5: Image of $\mathrm{CP} 6$ be sent to deploy the secondary payload that consists of a series of spring steel tapes. The data was intended to be used to guide the future design of an electrodynamic tether. [44]

For this case study we will be examining the primary mission for CP6. The secondary mission would have been a One-Shot mission.

\begin{tabular}{|l|c|}
\hline Parameter & Value \\
\hline Amount of Data collected & $3.54 \mathrm{MB}$ \\
Amount of Data Generated & $3.54 \mathrm{MB}$ \\
Mission Length & 6 months \\
Size & $1 \mathrm{U}$ \\
Transmitter Power & 500 milliwatt $(\mathrm{mW})$ \\
Network used & Full World \\
Decoder used & More dBs \\
\hline
\end{tabular}

Table 5.3: Parameters for the CP6 mission

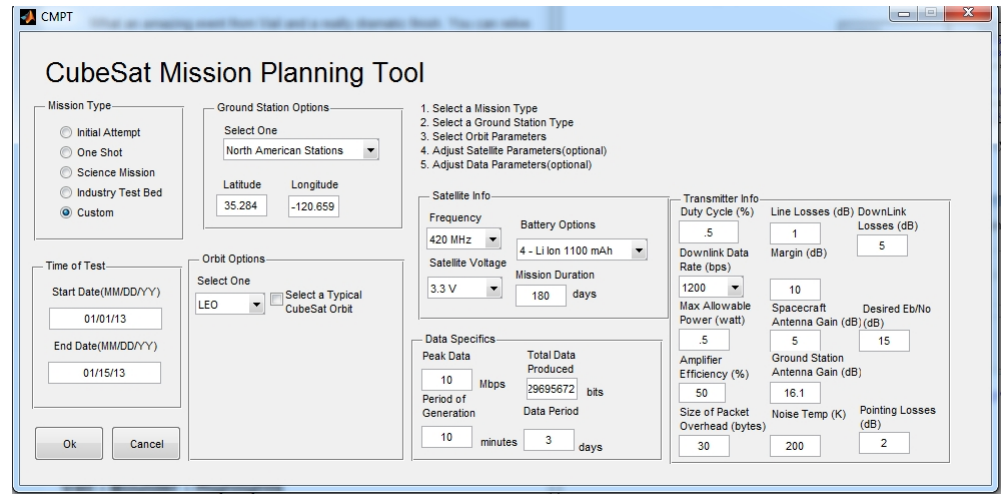

Figure 5.6: Screenshot of CP6 CMPT setup window 


\subsubsection{One Shot: CP5}

CP5 is a $1 \mathrm{U}$ CubeSat built by Cal Poly's PolySat Program. The payload is designed to test a scalable de-orbiting mechanism. [45] The mechanism consists of a miniature solar sail, similar to the ones used by NanoSail-D [46] or LightSail [31]. Once the sail is deployed observations will be made from the ground to determine the rate of degradation and other optical properties

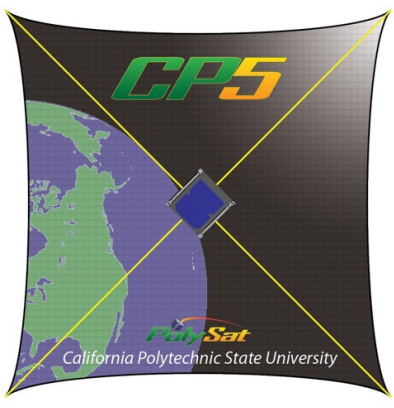

Figure 5.7: CP5 mission badge

of the sail.

\begin{tabular}{|l|c|}
\hline Parameter & Value \\
\hline Amount of Data collected & Not in Orbit - 340kilobyte (kB) per experiment \\
Amount of Data Generated & Not in Orbit - 340kB per experiment \\
Mission Length & 4 months \\
Size & $1 \mathrm{U}$ \\
Transmitter Power & $500 \mathrm{~mW}$ \\
Network used & Full World \\
Decoder used & More dBs \\
\hline
\end{tabular}

Table 5.4: Parameters for the CP5 mission

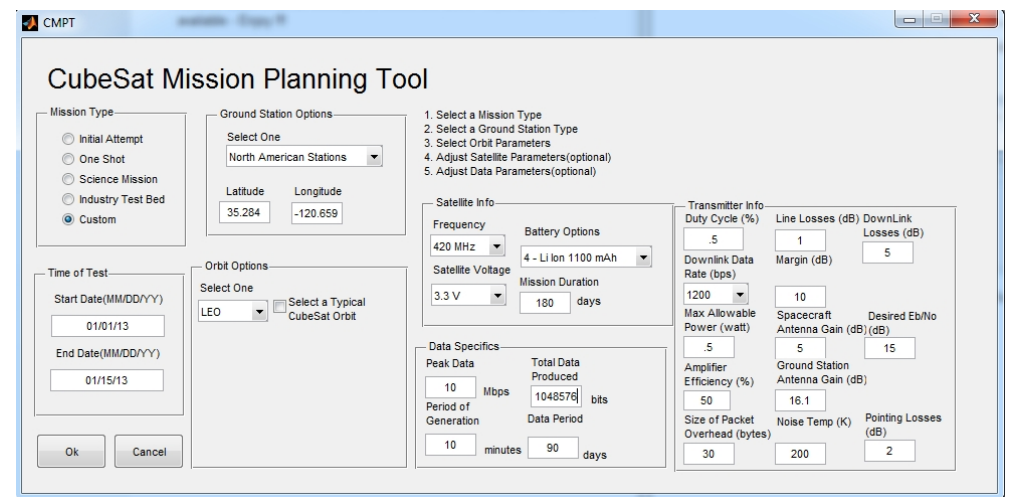

Figure 5.8: Screenshot of CP5 CMPT setup window 


\section{CASE STUDIES}

\subsubsection{Science: QuakeSat}

The QuakeSat mission demonstrated techniques to detect, record and downlink earthquake ELF emission data. QuakeSat demonstrated the feasibility of utilizing commercially available off the shelf parts for a reliable, short mission life satellite.

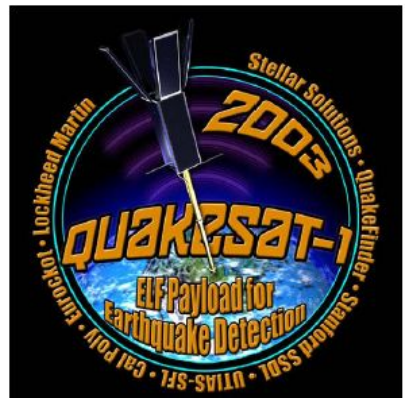

Figure 5.9: QuakeSat mission badge

\begin{tabular}{|l|c|}
\hline Parameter & Value \\
\hline Amount of Data collected & 1 gigabyte (GB) \\
Amount of Data Generated & $1 \mathrm{~GB}$ \\
Data generated per experimental period & $11 \mathrm{MB}$ \\
Mission Length & 4 months \\
Size & $3 \mathrm{u}$ \\
Transmitter Power & $1.4 \mathrm{Watts}$ \\
Network used & Single Station at Stanford University \\
Decoder used & No Decoder used \\
\hline
\end{tabular}

Table 5.5: Parameters for the QuakeSat mission

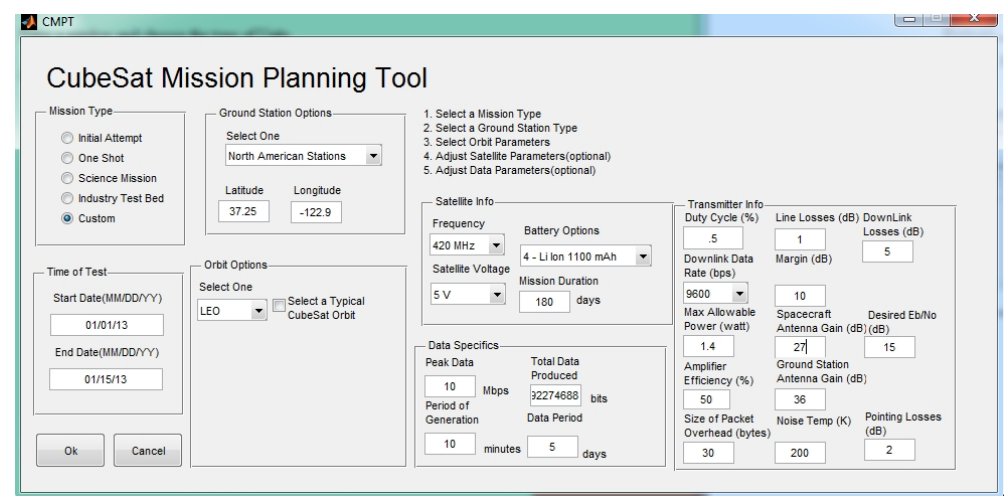

Figure 5.10: Screenshot of QuakeSat CMPT setup window 


\subsubsection{Science: RAX-2}

RAX is a joint venture between the University of Michigan and SRI International. Its primary mission objective is to study large plasma formations in the ionosphere, the highest region of our atmosphere. These plasma instabilities are known to spawn magnetic field-aligned irregularities (FAI), or dense plasma clouds known to disrupt communication between Earth and orbiting spacecraft. [27]

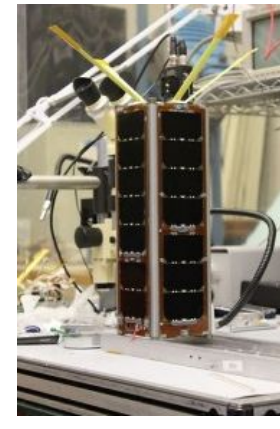

Figure RAX-2

\begin{tabular}{|l|c|}
\hline Parameter & Value \\
\hline Amount of Data collected & not finished with mission \\
Amount of Data Generated & not finished with mission \\
Data generated per experimental period & 1.2 GB of raw radar data on board in less than 1 hour [47] \\
Mission Length & 1 year \\
Size & $3 \mathrm{u}$ \\
Transmitter Power & 2.5 watt (W) on $437 \mathrm{MHz}[48]$ \\
Network used & Full World \\
Decoder used & RAX Ground Station Client \\
\hline
\end{tabular}

Table 5.6: Parameters for the RAX-2 mission

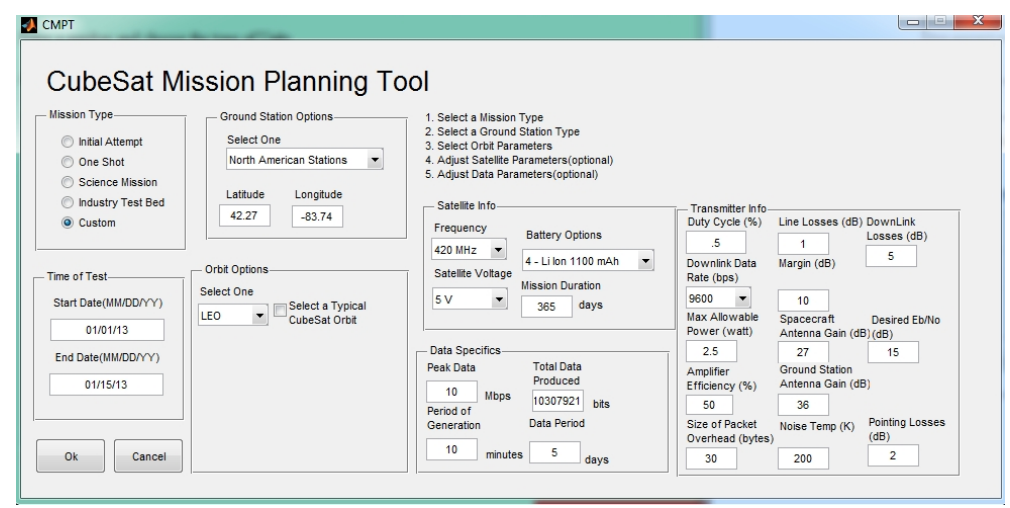

Figure 5.12: Screenshot of RAX-2 CMPT setup window 


\section{CASE STUDIES}

\section{$5.3 \quad$ Results}

This section will be a compare the missions in the real world cases section 5.2 and where possible this section will compare the GSI method, and the BC method of downlinking data. The comparsion uses the percent of data that was downlinked, the power density requirements, and finally the storage needs for each of these methods. 


\subsubsection{CP6}

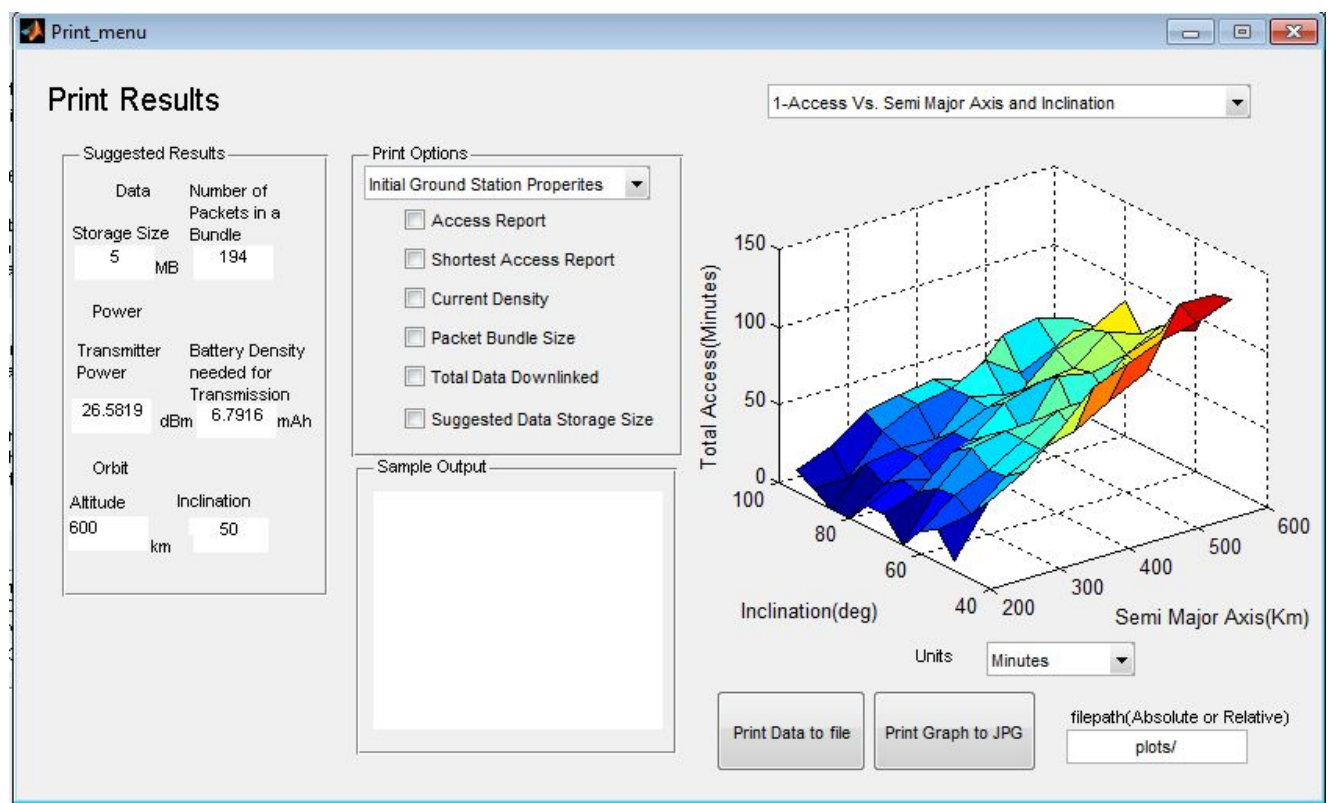

Figure 5.13: Screenshot of CP6 Print Screen

The small amount of data shows that the BC method will provide more opportunities to downlinking data. However, the amount of power required to operate use the $\mathrm{BC}$ method can be seen in figure $5.15 \mathrm{~b}$ is not a feasible option for a satellite such as CP6.

\begin{tabular}{|l|c|c|}
\hline & GSI & BC \\
\hline Percent Downlinked (\%) & 67.9 & 269 \\
Storage Size (MB) & 5 & 3.54 \\
Power Density (mW-hours ) & 41.7 & 1461.8 \\
Percent Battery (\%) & 15.4 & 1006 \\
\hline
\end{tabular}

Table 5.7: Comparsion values for GSI and BC methods of CP6 


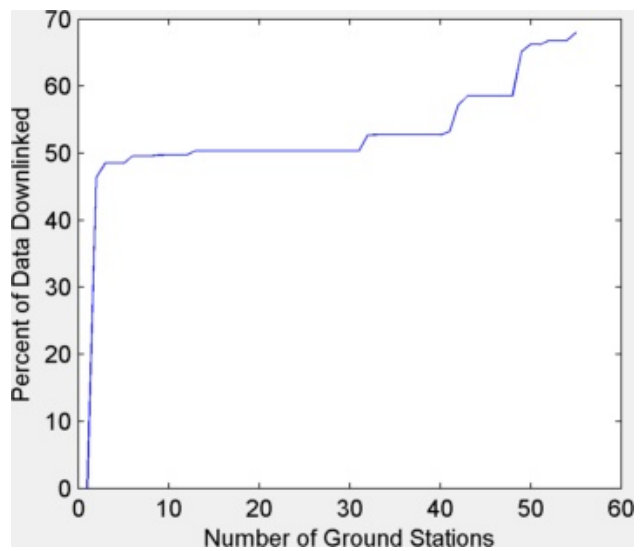

(a) GSI

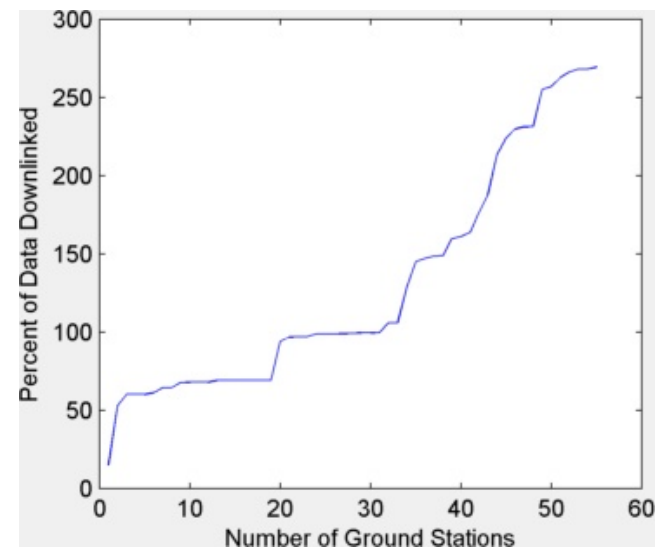

(b) $\mathrm{BC}$

Figure 5.14: Screenshots of Percent Downlinked Plots for CP6

\section{Percent of Data Downlinked with the GSI method}

The CP6 mission only produced 3.54 MB of data during the entire life time of the satellite. However, the time period for the data was decreased to three days to get a better understanding of the GSI method. As seen in figure $5.18 \mathrm{a}$, the maximum amount of data that was collected never reached the 100 percent mark. Had this test been accomplished during the early stages of design, the CP6 team would have been able to acknowledge that the ops plan needs to be adjusted to accommodate the rest of the data. One way of adjusting the ops plan to increase the percent of downlinked data would be to increase the data period to five or more days.

\section{Percent of Data Downlinked with the BC method}

The amount of data downlinked using the BC method (figure 5.18b) was more effective than the GSI method (figure 5.18a). This increase in downlinking capabilities with the BC method is attributed to the use of all the ground stations around the world. While the downlinking plan would be 
more complicated for the $\mathrm{BC}$ method, the downlinking capabilities are far superior using this method. In the early stages of design, reducing the duty cycle would not sacrifice any of the downlinking capabilities and potentially conserve battery capacity. 


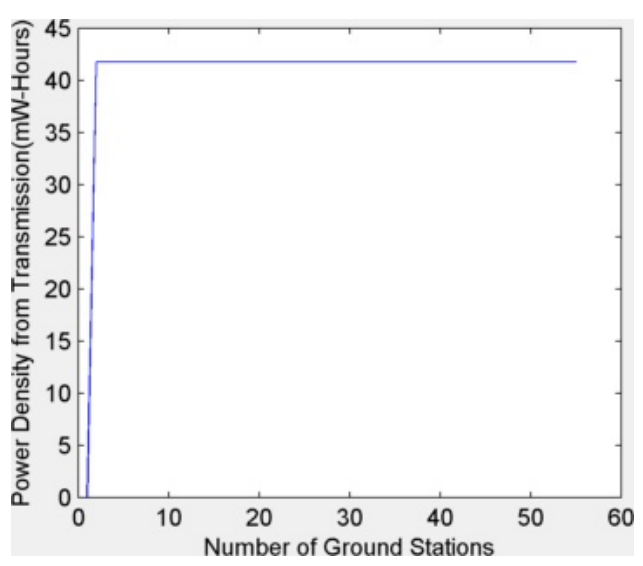

(a) GSI

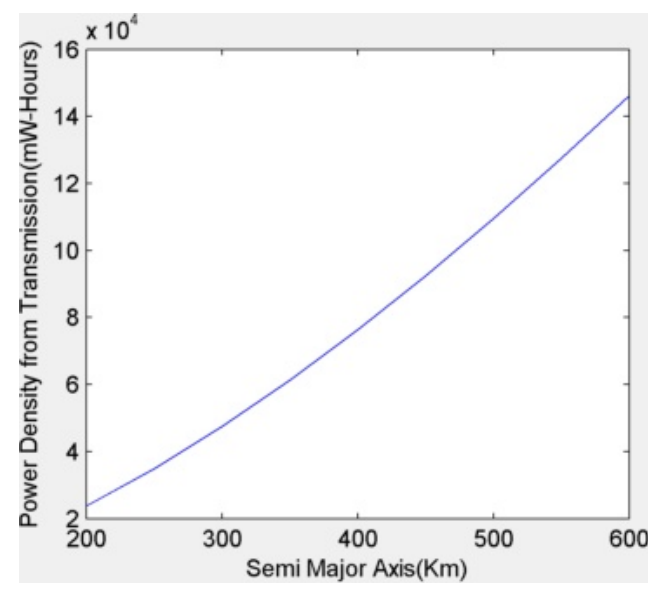

(b) $\mathrm{BC}$

Figure 5.15: Screenshots of the Power Density Plots for CP6

\section{Power Density of the GSI method}

The GSI method (figure 5.15a) has a power density around $40 \mathrm{~mW}$-Hours which translates to less than a percent of the battery being used. This information could help the CP6 team decide to increase the power out of the transmitter or even decrease the size of the batteries.

\section{Power Density of the BC method}

The nature of the BC method is not dependent on how many ground stations are available, however it is dependent on the time of the transmissions and the power required to downlink the data. This causes issues with the amount of that battery that will be used. The BC power usage would be for more than the battery is capable of supplying. One option would be to reduce the duty cycle and sacrificing some of the downlinking capability to ten percent or lower. 


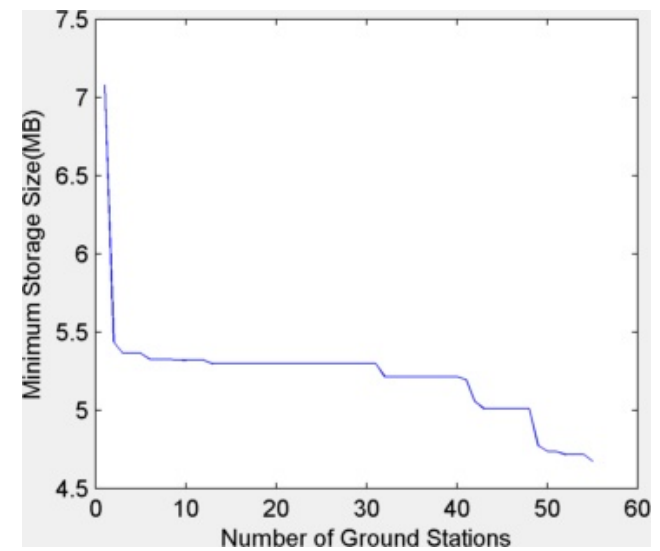

(a) GSI

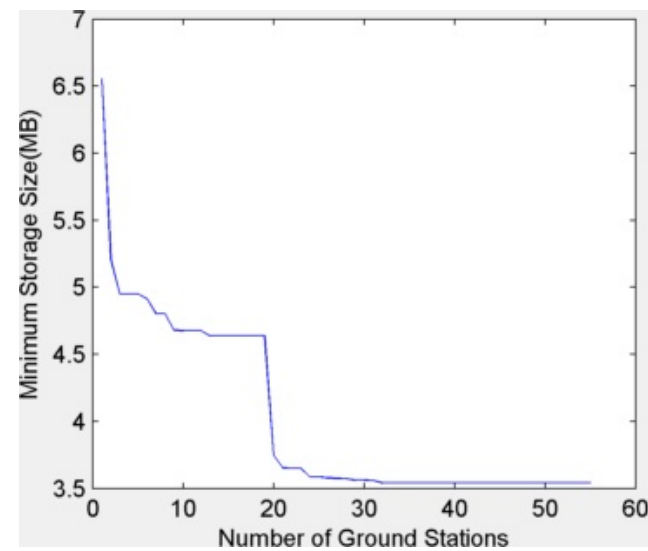

(b) $\mathrm{BC}$

Figure 5.16: Screenshots of the Storage Required Plots for CP6

\section{Storage Required for the GSI method}

In figure 5.16a the data storage required for CP6 remains low. The maximum storage size of $7 \mathrm{MB}$ is far below the $100 \mathrm{MB}$ of data storage the CP6 developers had included with CP6.

\section{Storage Required for the BC method}

Similar to the GSI downlink method, the amount of data that can be downlinked is enormous compared to the actual data produced, therefore the storage size should stay minimal.

\section{Conclusion}

The CP6 mission would benefit from using the GSI method over the BC method, because of the high power density required for the $\mathrm{BC}$ method. After running CMPT and examining figure 5.18a, the increase the data period to include more time would be helpful in completely the mission if the data period was three days. 


\subsubsection{CP5}

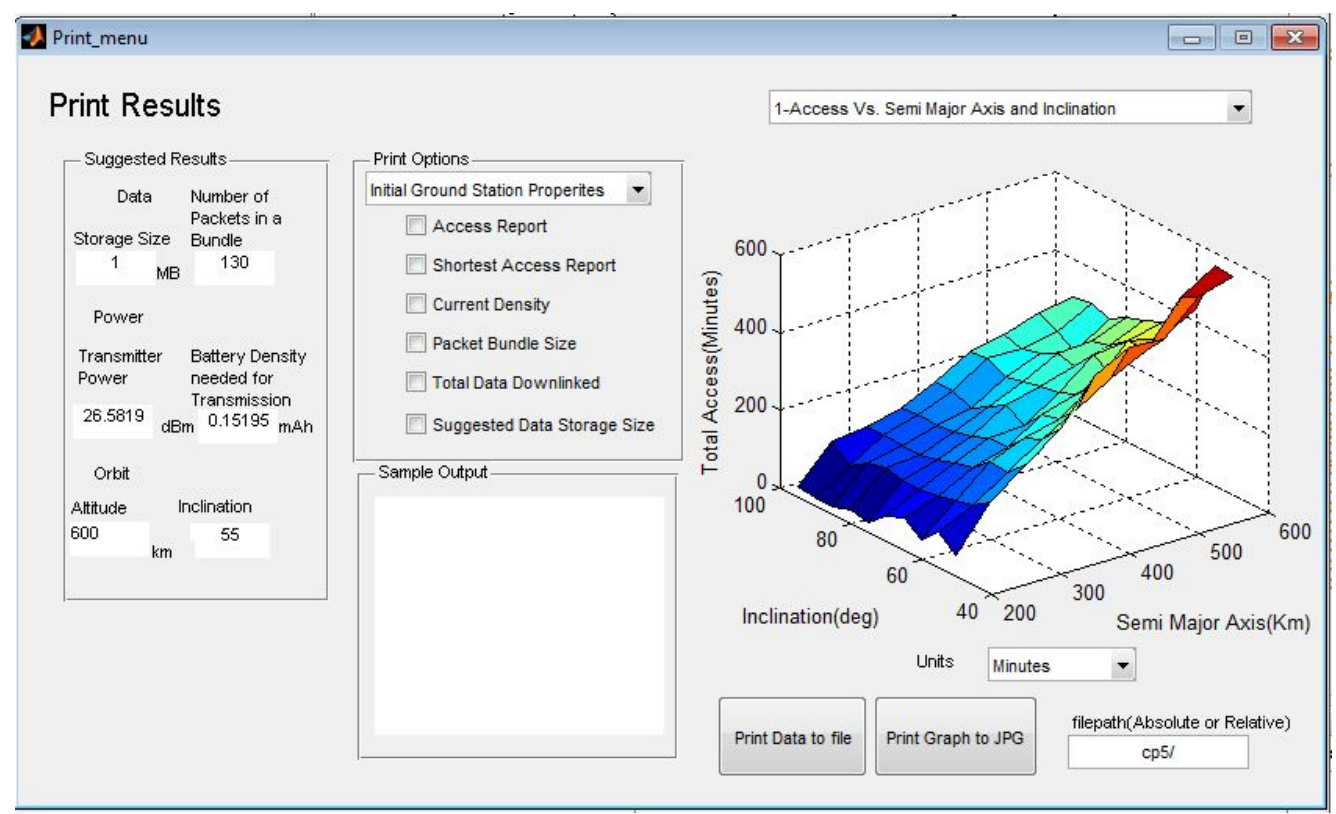

Figure 5.17: Screenshot of CP5 Print Screen

Similar to the CP6 test case, the data requirements for the CP5 mission is very small, downlink $340 \mathrm{kB}$ of data in 90 days. As the plots in the following pages will show, only the minimum is needed to complete this mission. It is important to note that although the mission was 90 days long, the test was long conducted for 14 days.

\begin{tabular}{|l|c|c|}
\hline & GSI & BC \\
\hline Percent Downlinked (\%) & 10,200 & 33,000 \\
Storage Size (MB) & 1 & 1 \\
Power Density (mW-hours ) & 28 & 146,000 \\
Percent Battery (\%) & .0035 & 1006 \\
\hline
\end{tabular}

Table 5.8: Comparsion values for the GSI and BC methods of CP5 


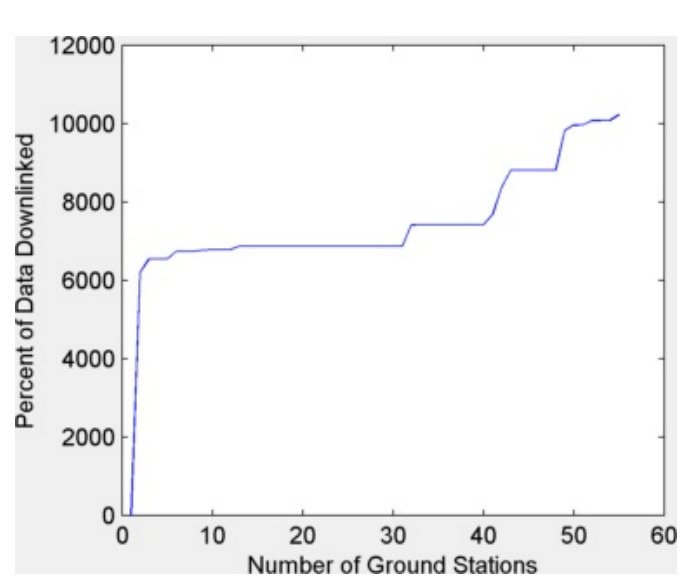

(a) GSI

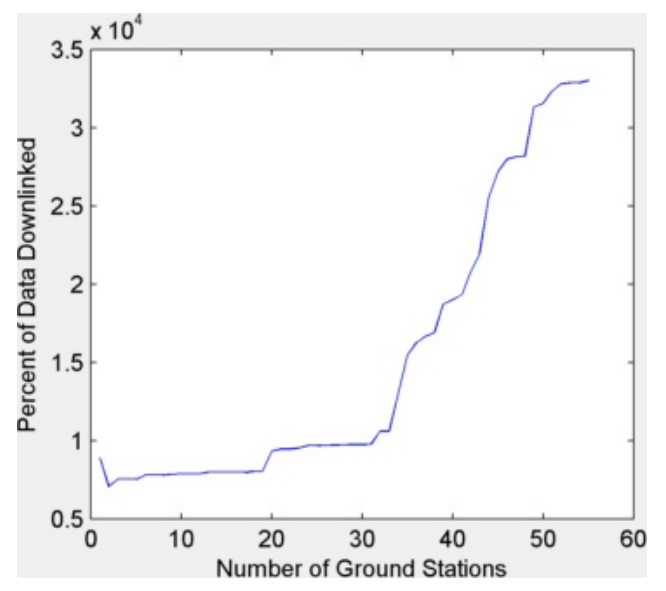

(b) $\mathrm{BC}$

Figure 5.18: Screenshots of Percent Downlinked Plots for CP5

\section{Percent of Data Downlinked with the GSI method}

The percent of data for this mission is enough to sustain operations. Even with the reduced mission life of 14 days, the downlinking capabilities are acceptable. One suggestion that could be made from figure $5.18 \mathrm{~b}$ is to reduce transmitter capabilities if battery usage was an issue such as decreasing the duty cycle.

\section{Percent of Data Downlinked with the BC method}

The BC method allows for downlinking capabilties far beyond the need of this mission and would not be used for this mission. 


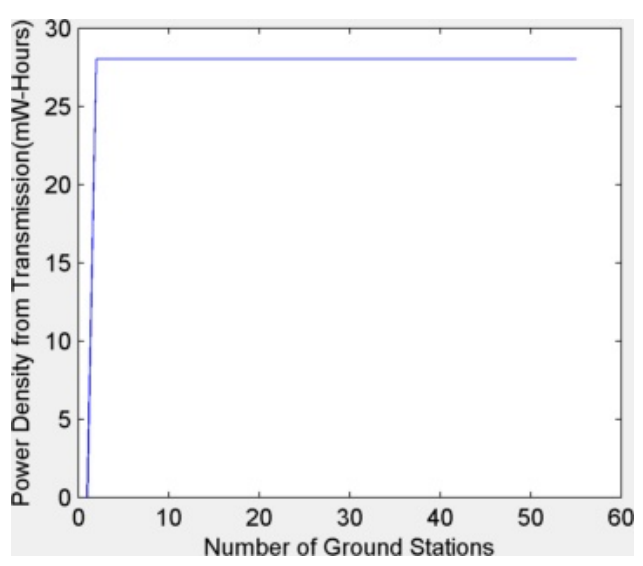

(a) GSI

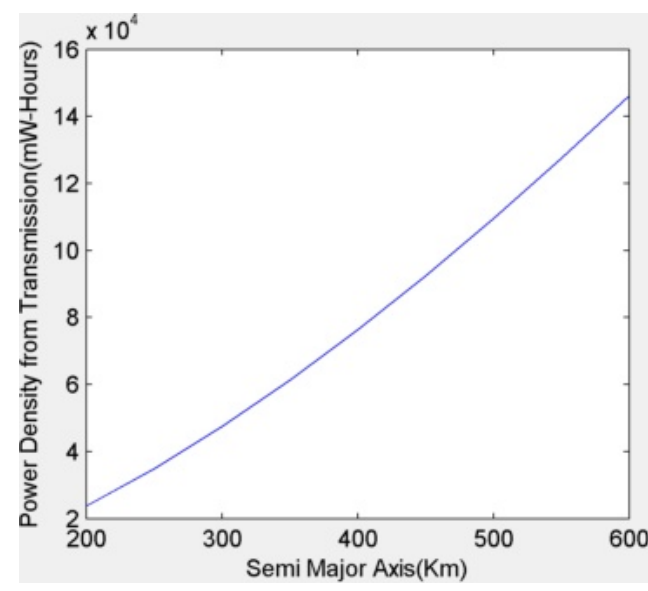

(b) $\mathrm{BC}$

Figure 5.19: Screenshots of the Power Density Plots for CP5

\section{Power Density of GSI}

The power density used with the GSI method is so small that it is almost negligible. In figure 5.19a the maximum power density used by the transmitter is less and $30 \mathrm{~mW}$ - hours, which translates to much less than one percent of the batteries capacity.

\section{Power Density of BC method}

The BC method of downlinking will always have a much larger need for power density. If this method of downlinking was used, the battery capacity would have to be greatly increased and the duty cycle would have to be decreased a substantial amount. 


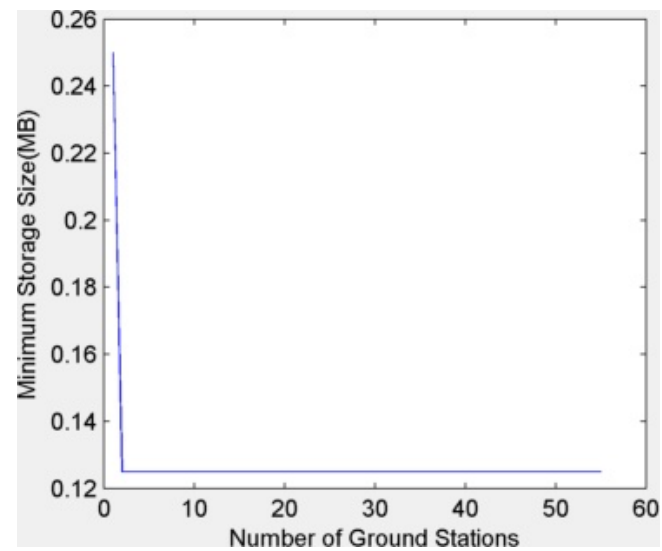

(a) GSI

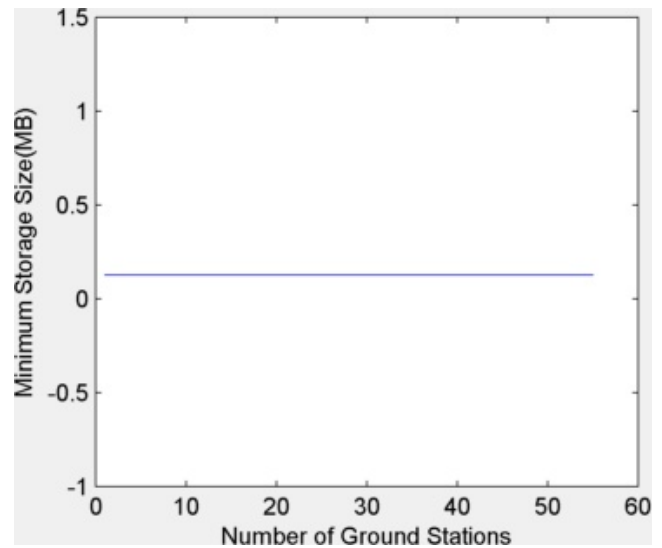

(b) $\mathrm{BC}$

Figure 5.20: Screenshots of the Storage Size Plots for CP5

\section{Storage Required for GSI}

For the GSI method CMPT would recommend only having the minimum amount of storage for data. Figure 5.20a confirms that the minimum requirement.

\section{Storage Required for BC method}

Similiar to the GSI method, figure 5.20b shows that CMPT would recommend only having the minimum amount of storage for data.

\section{Conclusion}

The CP5 mission would benefit from using the GSI method which would help reduce wear on the batteries. Since the amount of data that needs to be downlinked is so small, the need for the more downlinking opportunties is not warranted. 


\subsubsection{QuakeSat}

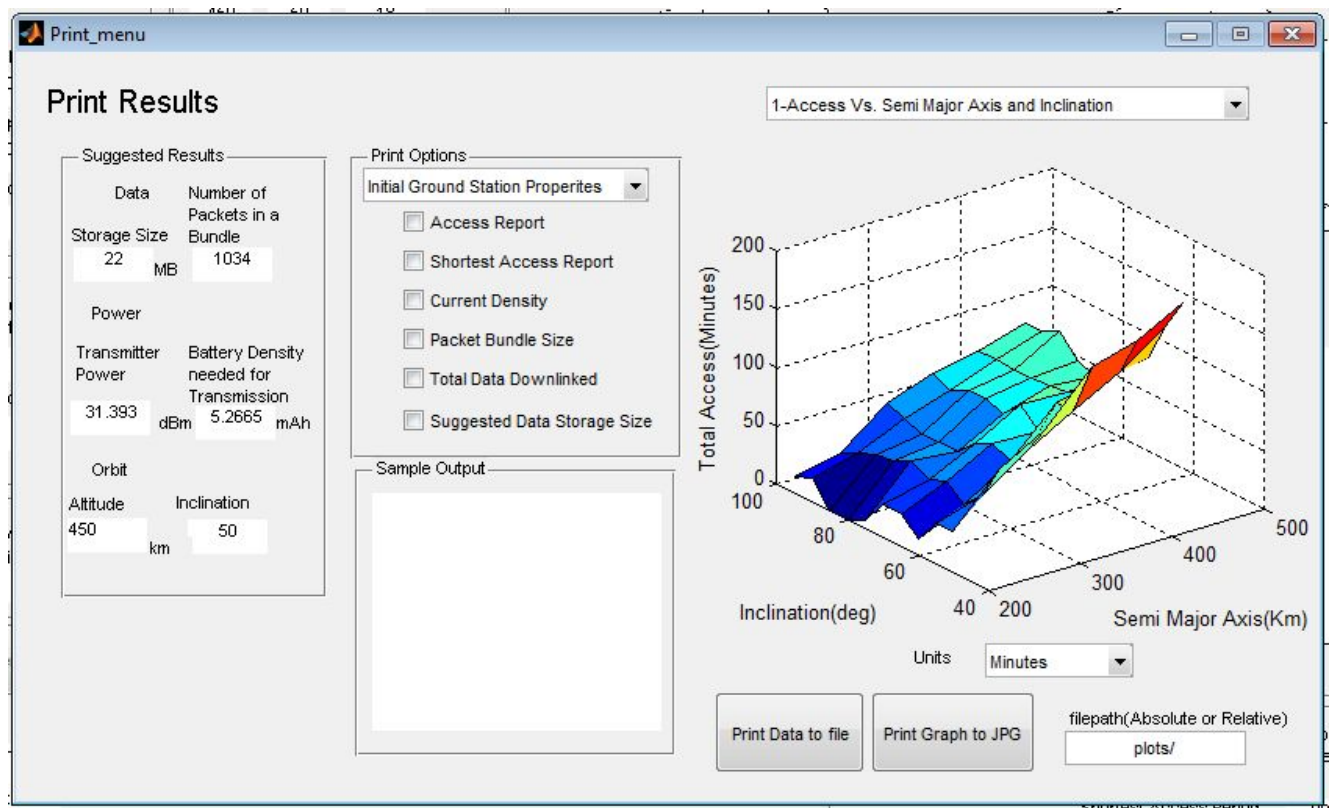

Figure 5.21: Screenshot of QuakeSat Print Screen

The QuakeSat mission shows an interesting look at the single ground station capabilities as well as looking at much larger discepanty in terms of the level of downlinking available. However, since QuakeSat uses only one ground station, the BC or GSI methods will not be examined. QuakeSat will be used in comparsion with the RAX mission to show the superior nature of using the entire world to downlink data.

\begin{tabular}{|l|c|}
\hline Parameters & Value \\
\hline Percent Downlinked (\%) & 32 \\
Storage Size (MB) & 18 \\
Power Density (mW-hours ) & 84.4 \\
Percent Battery (\%) & 5.8 \\
\hline
\end{tabular}

Table 5.9: The Results of the QuakeSat Mission 


\section{Shortest Access Periods}

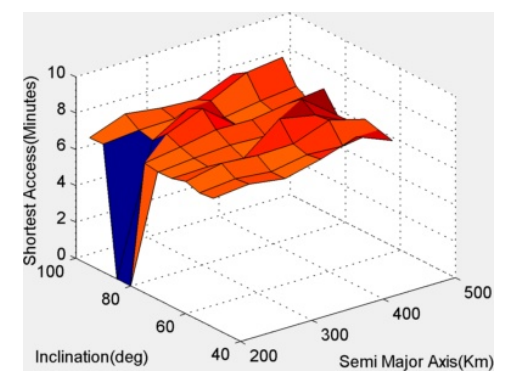

Figure 5.22: Screenshot of Shortest Access for QuakeSat

One of the major limiting factors of a single ground station is the lower access times. Notice all the access times in figure 5.22 are around 7 minutes long. In the next couple sections the figures look very similiar. The similiarity is a result of how CMPT use of the shortest access times to determine the amount of data downlinked, the percent downlinked and the power density. Section 4.4 can provide a more in depth understanding of this calculations.

\section{Percent of Data Downlinked with Ground Station Initiation}

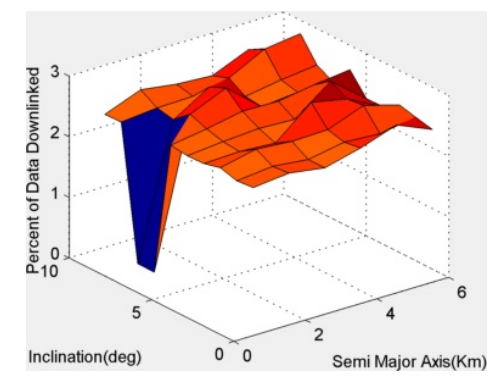

Figure 5.23: This plot represents the amount of data downlinked witha single station at Stanford University University.

As seen in figure 5.23, the amount of data that can be downlinked during the data period is extremely small with a maximum percent less 


\section{CASE STUDIES}

three percent. This will be a problem if this data is extremely time sensitive. Although time sensitivity is not in the scope of this thesis, the low percent downlinked can be a good indicator of needing a more robust communication system especially if this was early in the stage of CubeSat development.

\section{Power Density of Ground Station Initiation}

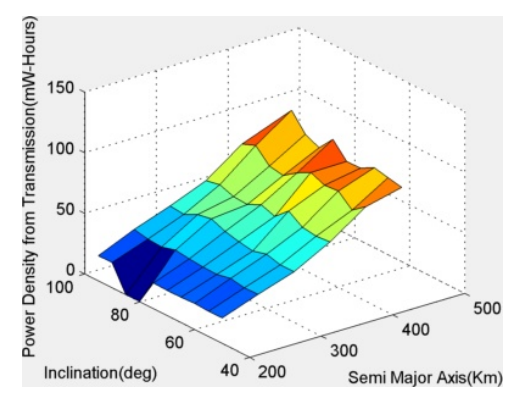

Figure 5.24: This plot represents the amount of the power density required to downlink the required data.

The low power density should be intitutive since QuakeSat was only downlinking over Stanford University with access times in the 6 to 8 minutes as seen in figure 5.22. This lower power density means that much more power can be used in the payload design which can then be used to increase the data period.

\section{Conclusion}

In 2003 when QuakeSat was launched, the amateur radio community had not began to capture data for the CubeSat community. If QuakeSat flew today, it would have definitely used a ground station network similiar to that of CP5 or CP6 to provide more downlinking opportunities. However, since the mission only had a single ground station available, after running 
CMPT it would be helpful to look at increasing the data rate or increasing the transmit power to increase the percent of the data that was downlinked. Using the plots provided in section 5.3.4, there can be a comparsion between the amount of data that can be downlinked using one ground station versus many ground stations around the world. While the most of the results are fairly obvious, that the amount of data downlinked was increased, one of the more interesting factors is that the power needed to downlink the data actual decreased. 


\section{CASE STUDIES}

\subsubsection{RAX-2}

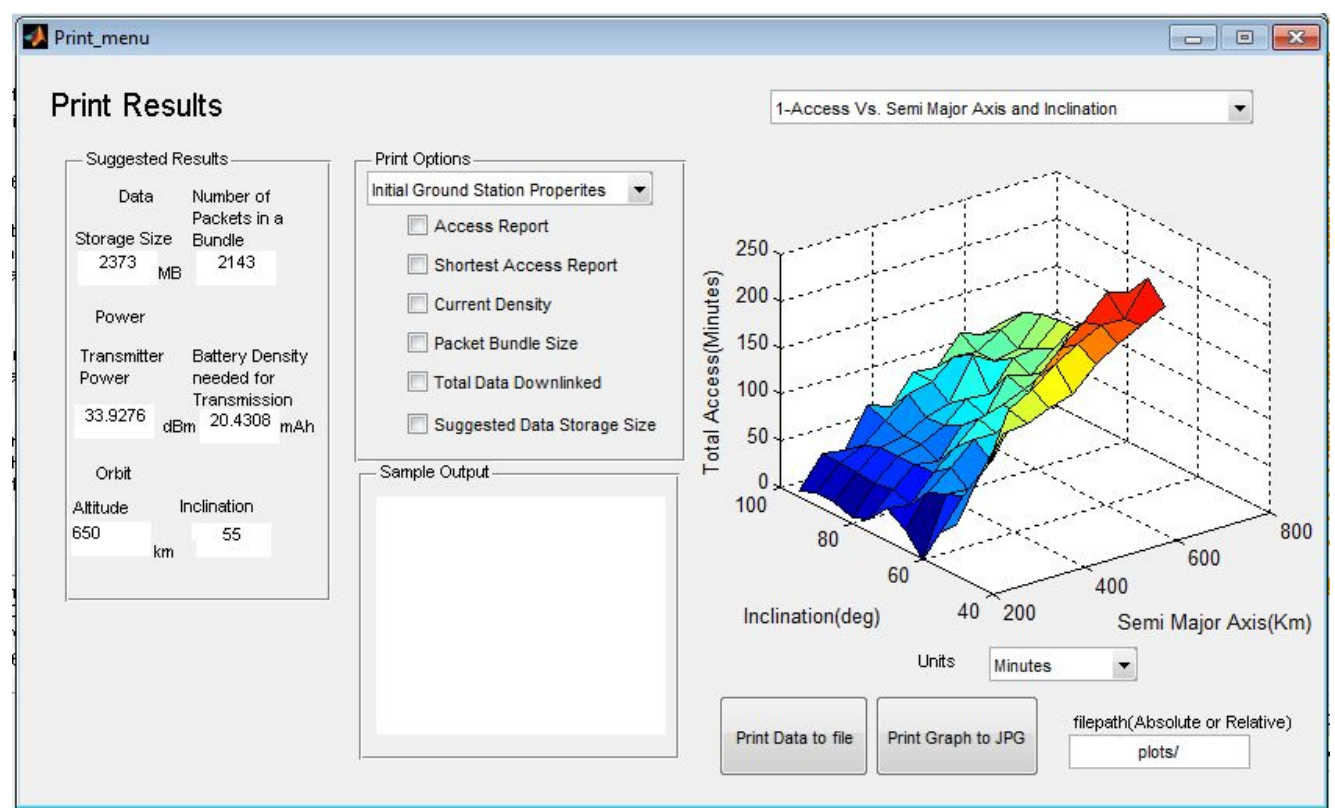

Figure 5.25: Screen Shot of RAX-2 Print Screen

\begin{tabular}{|l|c|c|}
\hline & GSI & BC \\
\hline Percent Downlinked (\%) & 6.9 & 16 \\
Storage Size (MB) & 2,373 & 2,260 \\
Power Density (mW-hours ) & 313.6 & 796,800 \\
Percent Battery (\%) & .46 & 3,622 \\
\hline
\end{tabular}

Table 5.10: Comparsion values for GSI and BC methods of RAX-2 


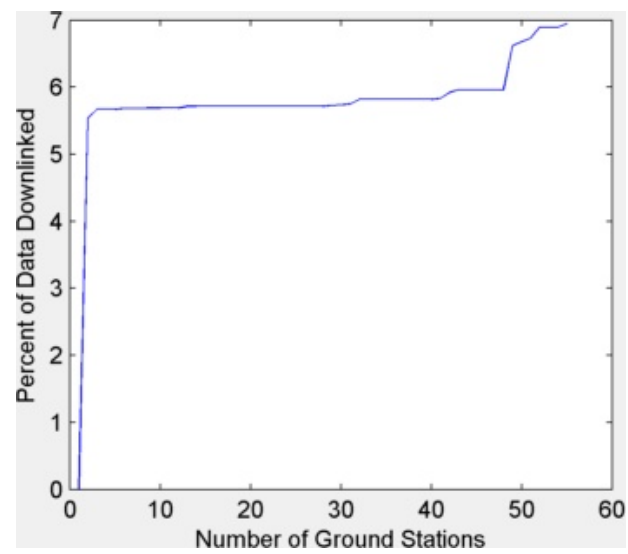

(a) GSI

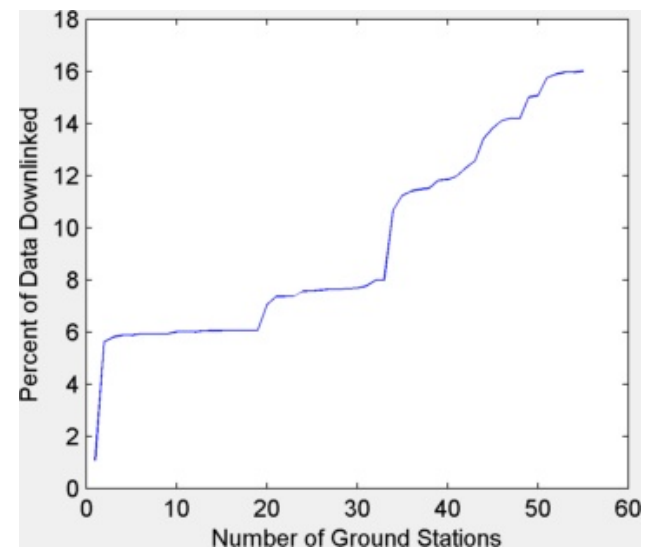

(b) $\mathrm{BC}$

Figure 5.26: Screenshots of Percent Downlinked Plots for RAX-2

\section{Percent of Data Downlinked with the GSI method}

In the GSI method, there is still a huge discrepancy in the amount of data that can be downlinked. In figure 5.26a, the maximum percent of data downlinked is around seven percent, which is far below anything useful for a mission.

\section{Percent of Data Downlinked with the BC method}

The BC method although not much better is still a improvement over the single station (figure 5.23) and the GSI method (figure 5.26a). However, downlinking 16 percent of the data is still not optimal and it would be recommend for this team to look into other methods to downlink their data. One of the ways that the RAX team overcame this issue was including a $2.4 \mathrm{GHz}$ transmitter that could operate at 115 kilobits per seconds (kbps) [35]. 


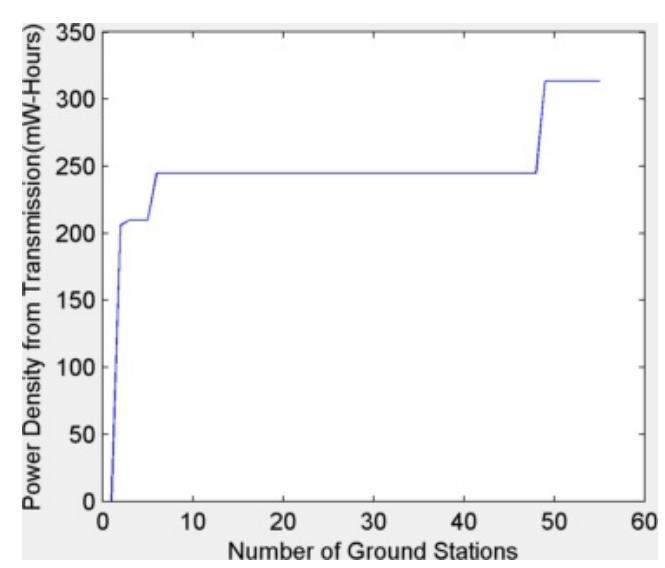

(a) GSI

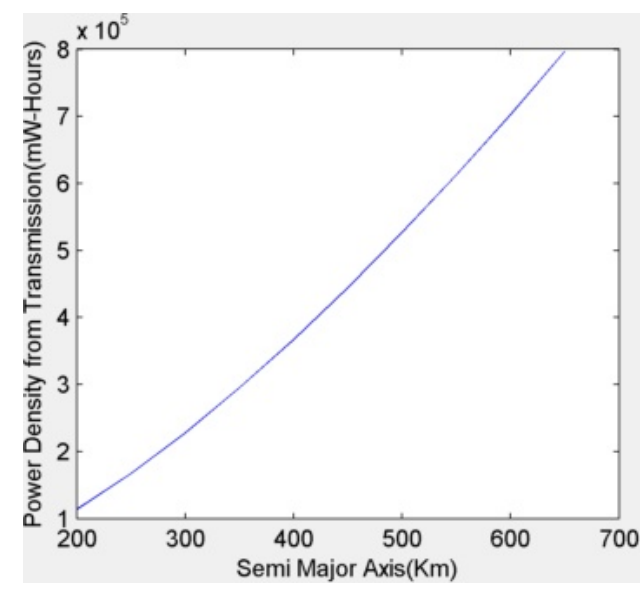

(b) $\mathrm{BC}$

Figure 5.27: Screenshots of the Power Density Plots

\section{Power Density of Ground Station Initiation for RAX-2}

As with previous uses of the GSI method, the amount of power used is much less than that of the $\mathrm{BC}$ method. The maximum power density for the GSI method would be less $314 \mathrm{~mW}$-hours which is still less than one percent of the batteries capacity. Although this value is still small these values should still be included in the power budget.

\section{Power Density of Broadcast method}

In figure $5.27 \mathrm{~b}$ the power density requirement is too large to be feasible. The power required for this method effectively negates its functionality. Major changes would have to be made to reduce the power required for this mission to be possible. 


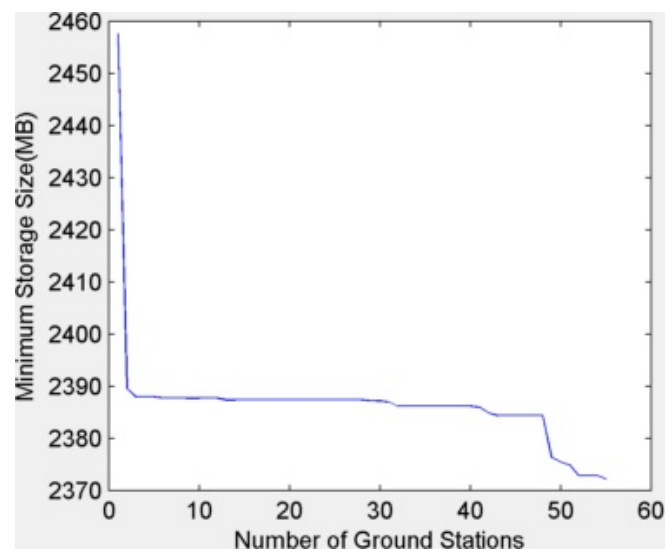

(a) GSI

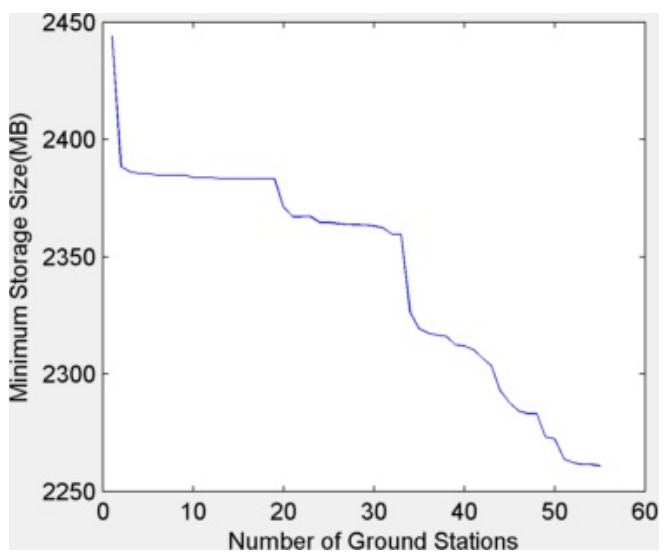

(b) $\mathrm{BC}$

Figure 5.28: Screenshots of the Storage Required Plots for RAX-2

\section{Storage Required for Ground Station Initiation}

In figure 5.28a the need for $2.5 \mathrm{~GB}(2460 \mathrm{MB})$ storage was anticipated given the amount of data that was orginally produced. While amount of storage needed does drop, the drop wont affect the size of storage needed.

\section{Storage Required for BC Method}

Similiar to the GSI method, the 2.5 GB (2460 MB) storage would definitely be a recommendation.

\section{Conclusion}

Many of the observations made with CMPT suggest that the use of the $\mathrm{BC}$ method is better suited for the use of a mission with an extremely large amount of data to downlink on a regular basis. However, using the GSI method while increasing the downlink data rate and power out of the transmitter will drastically improve downlinking capabilities and not scarfice the battery capacity. 
5. CASE STUDIES 


\section{6}

\section{Conclusion}

\subsection{Validation}

Three main sections of concern were validated using different techniques, such as power calculations with an industry standard link budget spreadsheet and using publications on downlinking specifications for past missions to compare with CMPT. These validations were used to determine the functionality of CMPT as an initial design tool.

\subsubsection{Link Budget Calculation}

Link Budget calculations were verified using Jan Kings Link Budget located at http://www.amsatuk.me.uk/. This link budget is the standard link budget used with amatuer radio communications and has been used many times for PolySat missions.

To validate the power equations, the values produced by CMPT were input into the link budget and then examined to see if the values matched. During this validation, CMPT produced results that were consistent with the link 
6. CONCLUSION

budget. 


\subsection{Validation}

\subsubsection{Single Station Mission}

To validate the functionality for single station missions, QuakeSat was used as a benchmark. QuakeSat published some documentation about downlinking capablities over the mission life. The QuakeSat team explained that on a good day the team could downlink $6 \mathrm{MB}$ [49]. CMPT can show that with a $100 \%$ duty cycle, QuakeSat will be downlinking 4.8 MB-7.2 MB depending on the given day or time period.

\subsubsection{Full World Mission}

\begin{tabular}{|c|c|l|l|l|}
\hline & CP6 mission Data & $\begin{array}{l}\text { With 100 percent } \\
\text { downlinking success }\end{array}$ & $\begin{array}{l}\text { CMPT GSI Mode (50 } \\
\text { \% D.C. ) }\end{array}$ & CMPT BC Mode \\
\hline Number of Packets & 1,969 & 25,649 & 3,880 & 39,004 \\
Total Downlinked(bits) & $3,150,400$ & $41,038,800$ & $7,946,240$ & $79,881,358$ \\
Total Downlinked(MB) & .3755 & 4.89 & .947 & 9.5226 \\
\hline
\end{tabular}

Table 6.1: Comparsion of CP6 Data and CMPT output

In table 6.1 there is a comparsion between the real world CP6 data and the values that CMPT produced. The values produced were in range of the values of the real world data. Although the values do not match exactly, they are in range of what should be anticipated. Many things play into the real world data that can not be simulated such as inefficient downlinking or operators not tracking the satellite. As an initial design tool, the simulation will provide a worst case situation that can be used to address uses in the design cycle. 


\subsection{Success of CMPT}

There were some general observations after running CMPT against different past missions. Overall, the use of $\mathrm{BC}$ method uses too much power to be effective and the GSI method while power effecient, was not always effective in downlinking enough data. The use of CMPT in the initial planning stages can provide useful suggestions to increase the effectiveness of a mision. One the most helpful components of CMPT was looking at the percent of downlink information, allowing a user to see how much data will be downlinked. This can then be used to analyze their ops plan and payload data production characteristics.

\subsection{Success Against CubeSat Case Studies}

Using CMPT against previous mission was a valuable excerise in gauging the usefulness of CMPT. However, at the present the suggests that CMPT proceduced can only be used to gauge future missions. One of the major successes of CMPT was showing the differences between the $\mathrm{BC}$ and the GSI methods. While it was clear that the BC method would provide higher amounts of downlinked data, the major drawback to this method was the battery density that was used was larger than the GSI method. As was shown in figure 5.15, the power density for CP6 was large for the BC method and inconsequential for the GSI method. Overall, CMPT was successful at providing details about the CubeSat missions and suggesting values that would allow for a further optimized system. The base cases provide a good reference for the initial stage of the design process. 


\subsection{Future Work}

There are many areas of CMPT that can be improved.

- Multiple Control Ground Station - Some of that work that would be of interest to expand upon is the development multiple command stations. This would greatly increase the ability of a Satellite operator to increase the downlinking capabilities of a CubeSat mission; while the only option available to the CubeSat community is the use of GENSO. However as stated in section 2.2.4 GENSO is not currently in a usable state. In the future, an open source and fully operational GENSO will be available to the community.

- Power Generation Simulation - To increase the usablity of CMPT it would be helpful to add power generation simulations to CMPT. This might include solar cell characterization or adding more components to the understanding of the system. Solar cell characterization might allow for multiple solar cell configurations and estimate orbit performance. This might also include adding components to understand the system better such as attitude control, processors and non-transmitter electronics.

- Expand the Payload inputs - The current version of CMPT only inputs the data production requirements of the payload however, understanding the power and station keeping requirements of a payload could dramatically improve the CubeSat model.

- Develop an STK alternative with MATLAB or C - During development of CMPT it became apparent that one of the limiting 


\section{CONCLUSION}

factors to the mobility of CMPT was its dependency on STK for orbit propagation. Part of the decreased mobility was STK's inabilty to interface with MATLAB on a 64 bit system. This might be fixed in future releases of STK. Another justification for finding an alternative is the need for a STK license which can be difficult to obtain for non educational institutions. This may happen in steps that might start with doing simple orbit propagations and then add ground station capabilites and further incorparating the sun to understand power capabilities.

- Dynamic Access Periods - Real Time analysis of the best option for downlinking packets would be an incredibly helpful improvement to CMPT. This might be accomplished by having a real time mode that runs STK or another orbit propagator to determine how long the next access period will be and recommend the correct packet bundle size for that pass. This improvement would greatly increase the functionality of CMPT as well as greatly improve downlinking capabilites of a CubeSat mission.

- Model-based System Engineering - One of the ways that this thesis could be expanded upon in future versions is to incorprate Cal Poly's Model-based System Engineering program Horizon. This C based program can help to determine orbits as described in Orbital Propators for Horizon Simulation Framework [50] which would elimanate the need for STK and speed up the simulations. 


\section{References}

[1] Bob Bruninga. Automatic Packet Reporting System. xvii, 9

[2] J. Taylor W. Beech, D Nielsen. AX.25 Link Access Protocol for Amateur Packet Radio, Jul 1998. xviii, 9, 45, 66

[3] Wiley J. Larson James R. Wertz. Space Mission Analysis And Design, 8. Third edition. xviii, xix, xx, xxi, 6, 46, 47, 48, 62, 64

[4] Howard D. Curtis. Orbital Mechanics for Engineering Students. Elsevier, 2nd edition. xxii

[5] D. Cuddleback. MoredBs, Jan 2010. xxii, 8

[6] CubeSat ELaNa III Launch on NPP Mission, Oct 2011. xxii

[7] STK. xxiii, 12, 15

[8] MAST. xxiv, 4

[9] K. Leveque B. Klofas, J. Anderson. A Survey of CubeSat Communication Systems, Nov 2008. 3, 19

[10] M. Ertan Umit and Alim Rustem Aslan. Lessons Learned: ITUpSAT1, Feburary 2011. 4 
[11] Dontchev K. Heywood M. Ramos R. Freyberg B. Wilson T. Pingree P. Saca F. Gilchrist B. Gallimore A. Cutler J. Bekker D., Werne T. A CubeSat Design to Validate the Virtex-5 FPGA for Spaceborne Image Processing. IEEEAC paper 1246, 2010. 4

[12] C. S.; Bust G. S.; Swenson C.; Barjatya A.; Larsen M. F.t Crowley, G.; Fish. Dynamic Ionosphere Cubesat Experiment (DICE). American Geophysical Union, Fall Meeting 2009, 2009. 4

[13] Elaine Caday-Eames. small box, big potential Boeing engineers think outside the cube in satellite development, Sep 2006. 4, 7, 28, 35

[14] J. Bellardo G. Manyak. PolySats Next Generation Avionics Design. 2011 Fourth IEEE International Conference on Space Mission Challenges for Information Technology, Aug 2011. 5

[15] Robert Cannon. ELaNa: Educational Launch of Nanosatellites, Feb 2011. 5

[16] Thomas H. White. Early United States Radio History, Aug 2005. 6

[17] Constitution of the International Amateur Radio Union, May 1989. 6

[18] D. Jansson K. Baker. Space Satellites from the World's Garage-The Story of AMSAT. Aerospace and Electronics Confer- 
ence, 1994. NAECON 1994., Proceedings of the IEEE 1994 National, May 1994. 6

[19] Hans van de Groenendaal. AmateuR RADiO SATELLITES INFORMATION FOR DEVELOPERS OF SATELLITES PLANNING TO USE FREQUENCY BANDS ALLOCATED TO THE AMATEUR-SATELLITE SERVICE, Oct 2006. 7, 39, 111

[20] R. Funase E. Takei Y. Nakamura M.i Nagai A. Enokuchi C. Yuliang K. Nakada Y. Nojiri F. Sasaki T. Funane T. Eishima S. NAKasuka. Technology demonstration on University of Tokyo's pico-satellite XI-V and its effective operation result using ground station network. Acta Astronautica, 61(78):707-711, 2007. 7

[21] James Cutler Julian Mann, August 2008. [link]. 8, 45

[22] B.M. ZiELINSKI. Effective transmission speed in AX.25 protocol, may 2009. 9

[23] A. Gonzalez. Genso. 10

[24] A. Fox J. Cutler, P. Linder. A FEDERAted GROUND STATION NETWORK, Aug 2002. 10

[25] Kostas Konstantinidis. CubeSat: a Review, Dec 2010. 19

[26] J. BLuCK. GeneSat-1. 21, 27

[27] Rax Mission Overview. 23, 27, 79 
[28] Jake A. Schaffner. The Electronic System Design, Analysis, Integration, and Construction of the CalPoly State University CP1 CubeSat, August 2002. 25

[29] H. Kayal, F. Baumann, K. Briess, and S. Montenegro. BEESAT: A Pico Satellite for the On Orbit Verification of Micro Wheels, june 2007. 25

[30] Maurice Borgeaud, Nomy Scheidegger, Muriel Noca, Guillaume Roethlisberger, Fabien Jordan, Ted Choueiri, and Nicolas Steiner. SwissCube: The First Entirely-Built Swiss Student Satellite with an Earth Observation Payload, 2010. 25

[31] LightSail-1. 28, 77

[32] Ground Station Survey Results. 35

[33] QuakeSat Lessons Learned: Notes from the Development of a Triple CubeSat. 40

[34] Hermes Power (EPS). 40

[35] The Radio Aurora EXplorer CubeSat Electrical Power System Overview. 41, 95

[36] R. Funase Y. Nakamura A. Enokuchi, M.i Nagai and S. NAKASUKA. REMOTE SENSING BY UNIVERSITY OF TOKYOS PICO-SATELLITE PROJECT PRISM, Nov 2004. 42 
[37] R. Brennan E. Galgana P. Gangar A. Kemis N. Lutzenhiser K. Moravec S. Mzali Z. Nazari J. Ross M. Bernhardt, A. Borth and E. Shin-White. RTICC Rapid Terrestrial Imaging CubeSat Constellation, June 2009. 43

[38] J. McNair P. Muri, O.i Challa. Enhancing Small Satellite Communication Through Effective Antenna System Design, 2010. 45

[39] S-Band Patch Antenna. 45

[40] Uhf/Vhf/S Band Ground Station. 45

[41] B. Sklar. DIGITAL COMMUNICATIONS Fundamentals and Applications. Second edition. 46

[42] University of Tokyo CubeSat Project Critical Design Review, April 2001. 48

[43] Jan King. AMSAT / IARU Annotated Link Model System, Nov 2007. 62, 63, 64

[44] CP6 At A Glance. 76

[45] CP5 At A Glance. 77

[46] Brian Dunbar. NASA's Nanosail-D 'Sails' Home - Mission Complete, Jan 2012. 77

[47] J. Cutler H. Bachcivan. Radio Aurora Explorer: Mission science and radar system. RADIO SCIENCE, VOL. 47, April 2012. 79 
[48] REMOTE SENSING BY UNIVERSITY OF TOKYOS PICO-SATELLITE PROJECT PRISM, Sept 2009. 79

[49] C. Dunson J. Doering L. Demartini P. Clarke L. Franklin Dr. J. Seelbach J. Flagg M. Klenk V. Safradin J. Cutler S. Flagg, T. Bleier. Using Nanosats as a Proof of Concept for Space Science Missions: QuakeSat as an Operational Example, 2004. 101

[50] Mitra Farahmand. ORBITAL PROPAGATORS FOR HORIZON SIMULATION FRAMEWORK, August 2009. 104 


\section{Appendix A}

\section{Procedures for Acquiring}

\section{Amateur Frequency License}

Note: This is only a guide and should only be used as reference.

Note: For the most up to date information on frequency allocation visit:

IARU Information

1. A licensed amatuer radio operator that will act "solely with a personal aim and without percuniary interest" [19] must submit the paperwork.

2. Complete the IARU Coordination Request in the following section.

3. Send the Request to IARU Satellite Coordinator's Email

4. Once you have approval and a frequency from the IARU email the following documents to Joseph Hill at the FCC.

- Freq Authorization from the IARU

This should be sent to you after authorization from the IARU

- Satellite Summary

A template is included in the following pages 


\section{A. PROCEDURES FOR ACQUIRING AMATEUR FREQUENCY LICENSE}

- Debris Assesment Survey Output

The Debris Assessment software is available here.

- Satellite Link Budget

- Space Cap Database

Information on How to use the Space Cap Program and where to download the software is available here. 
A.1 IARU Coordination Request Form

\section{A.1 IARU Coordination Request Form}




\section{The International Amateur Radio Union}

Since 1925, the Federation of National Amateur Radio Societies Representing the Interests of Two-Way Amateur Radio Communication

\section{AMATEUR SATELLITE FREQUENCY COORDINATION REQUEST ${ }^{1}$}

1. Self coordination. For over 100 years, amateur radio operators have maintained an effective tradition of self-regulation. Amateurs are expected to coordinate their use of frequencies. (None of us has a right to use any particular frequency.) Coordination of many terrestrial stations, repeaters and beacons, for example, usually works well through IARU member national societies and local coordinating committees.

2. Coordinating satellites. Amateur radio satellites present a special problem because satellites have global effect. Only a global frequency coordination system can work. Uncoordinated satellites will cause harmful interference to stations around the world and receive interference from them — which could result in mission failure.

Coordination serves everyone's best interests!

\section{Coordination procedure.}

a. Frequency coordination for amateur radio satellites is provided by the IARU through its Satellite Advisor, a senior official appointed by the IARU Administrative Council, its top policymaking body. The IARU Satellite Advisor is assisted by an Advisory Panel of qualified amateurs from all three IARU Regions. (Similar to ITU Regions.)

b. In all other satellite services, frequency coordination is a mandatory process through the ITU Radiocommunication Bureau (BR). The procedure includes notification of all administrations (RR Article 11) and coordination with all administrations (RR Article 9) using BR publications and procedures.

c. IARU strongly recommends that you work with your administration and encourage them to notify amateur-satellite service stations using the Article 11 procedure. This way, all administrations will see more clearly the value of the amateur-satellite service. (Help with the notification process will be provided in a separate document.)

\section{Getting Help.}

a. Start by reading Amateur Radio Satellites, an IARU paper. You will find explanations and interpretations of Treaty provisions. IARU satellite frequency coordination follows

\footnotetext{
${ }^{1}$ Terms used here are defined in the IARU paper, Amateur Satellites. A PDF version is available at: http://www.iaru.org/satellite/IARUSATSPEC REV15.6.pdf.
} 
these interpretations. Download the latest version from: http://www.iaru.org/satellite/sat-freq-coord.html.

b. Discuss your project with the national amateur radio society of your country and your national AMSAT organisation, if there is one. They may be able to assist you in a variety of ways.

c. Use information available on-line.

i. For a list of national amateur radio societies (Member Societies of IARU), see: http://www.iaru.org/iaru-soc.html.

ii. For a list of amateur satellite organisations, see: http://www.amsat.org/amsatnew/links/.

iii. A link budget spread sheet is at: http://www.amsat.org.uk/iaru/spreadsheet1.asp.

iv. Check frequencies of currently operating satellites at: http://www.amsat.org/amsat-new/satellites/. Check on coordinated and other planned amateur satellites at: http://www.amsat.org.uk/iaru/.

v. If you need help understanding the requirements or completing the coordination request, ask the Satellite Advisor or a Panel Member.

5. When to make the frequency coordination request. Make your frequency coordination request as far in advance as possible. Remember, coordination takes account of your own needs and the needs of others. Receiving coordination early enough makes design and construction easier and less expensive. In any event, be sure to make your request while it is still possible to change operating frequencies in response to the Satellite Advisor's recommendations.

6. Who makes the frequency coordination request? The prospective space station licensee must make the coordination request, as that person will be responsible for space station transmitter operations.

7. Where to send your frequency coordination request. Send frequency coordination requests to the IARU Satellite Advisor by e-mail to satcoord@iaru.org with a copy to wozane@gmail.com.

8. What will happen? The IARU Satellite Advisor will make recommendations to the licensee concerning plans based upon all available information and advice from the Satellite Advisory Panel. His goal is to help you and your project to succeed. Application status will be published at: http://www.amsat.org.uk/iaru/. When the process is complete, the licensee will receive a coordination letter with detailed information.

\section{VERY IMPORTANT!}

1. Submit only the request form; do not send these instructions, please. 
2. Name the electronic document you submit with the name of the proposed satellite followed by the submission date. Example: if the name before launch is Newsat A and the document is submitted in November 2009, the document file name should be: "newsata_nov2009.doc."

3. Indicate in your request form the URL's for pictures, sketches, drawings, and other pertinent information.

4. Indicate whether or not you feel that the proposed operation in the amateursatellite service is consistent with the radio regulations as interpreted by the IARU Satellite Advisor. If not, please, explain your interpretation of the radio regulations.

5. Licensee, please, sign and date the form.

\section{— detach instructions, please -}




\section{The International Amateur Radio Union}

Since 1925, the Federation of National Amateur Radio Societies

Representing the Interests of Two-Way Amateur Radio Communication

\section{AMATEUR SATELLITE FREQUENCY COORDINATION REQUEST}

(Make a separate request for each space station to be operated in the amateur-satellite service.)

\section{Administrative information:}

\begin{tabular}{|c|c|c|}
\hline 0 & \multicolumn{2}{|l|}{ DOCUMENT CONTROL } \\
\hline $0 \mathrm{a}$ & Date submitted & \\
\hline $\mathrm{Ob}$ & Expected launch date & \\
\hline $0 \mathrm{c}$ & Document revision number & \\
\hline 1 & \multicolumn{2}{|l|}{ SPACECRAFT (published) } \\
\hline $1 \mathrm{a}$ & Name before launch & \\
\hline $1 \mathrm{~b}$ & Proposed name after launch & \\
\hline $1 \mathrm{c}$ & Country of license & \\
\hline 2 & \multicolumn{2}{|c|}{ LICENSEE OF THE SPACE STATION (published) } \\
\hline $2 a$ & First (given) name & \\
\hline $2 b$ & Last (family) name & \\
\hline $2 c$ & Call sign & \\
\hline $2 d$ & Postal address & \\
\hline $2 e$ & $\begin{array}{l}\text { Telephone number (including } \\
\text { country code) }\end{array}$ & \\
\hline $2 f$ & $\begin{array}{l}\text { E-mail address (licensee will be } \\
\text { our point of contact and receive all } \\
\text { correspondence) }\end{array}$ & \\
\hline $2 g$ & Skype name (if available) & \\
\hline $2 \mathrm{~h}$ & $\begin{array}{l}\text { Licensee's position in any } \\
\text { organisation referenced in item } 3 a \text {. }\end{array}$ & \\
\hline $2 \mathrm{i}$ & $\begin{array}{l}\text { List names and e-mail addresses } \\
\text { of additional people who should } \\
\text { receive copies of correspondence. }\end{array}$ & \\
\hline 3 & $\begin{array}{l}\text { ORGANISATIONS (published) - } \\
\text { organization }\end{array}$ & mplete this section for EACH participating \\
\hline $3 a$ & Name of organisation & \\
\hline $3 b$ & Physical address & \\
\hline $3 c$ & Postal address & \\
\hline $3 d$ & $\begin{array}{l}\text { Telephone number (including } \\
\text { country code) }\end{array}$ & \\
\hline $3 e$ & E-mail address & \\
\hline $3 f$ & Web site URL & \\
\hline $3 g$ & National Amateur Radio Society & \\
\hline
\end{tabular}




\begin{tabular}{|c|l|l|}
\hline & (including contact information) & \\
\hline $3 \mathrm{~h}$ & $\begin{array}{l}\text { National Amateur Satellite } \\
\text { organisation (including contact } \\
\text { information) }\end{array}$ & \\
\hline $3 \mathrm{i}$ & $\begin{array}{l}\text { Have you involved your National } \\
\text { Amateur Satellite organization } \\
\text { and/or National Amateur Radio } \\
\text { Society? Please, explain. }\end{array}$ & \\
\hline
\end{tabular}

\section{Space station information:}

\begin{tabular}{|c|c|}
\hline 4 & SPACE STATION (published) \\
\hline $4 a$ & $\begin{array}{l}\text { Mission(s). } \\
\text { Describe in detail what the space } \\
\text { station is planned to do. Use as } \\
\text { much space as you need. }\end{array}$ \\
\hline $4 b$ & $\begin{array}{l}\text { Planned duration of each part of } \\
\text { the mission. }\end{array}$ \\
\hline $4 c$ & $\begin{array}{l}\text { Proposed space station } \\
\text { transmitting frequency }{ }^{2} \text { plan. } \\
\text { List for each frequency or } \\
\text { frequency band: } \\
\rightarrow \text { frequency or frequency band } \\
\text { (e.g. } 435-438 \mathrm{MHz}) \\
\rightarrow \text { output power } \\
\rightarrow \text { ITU emission designator }^{3,4} \\
\rightarrow \text { common description of the }_{\text {emission }}^{5} \\
\rightarrow \text { antenna gain and pattern } \\
\rightarrow \text { attitude stabilisation, if used }\end{array}$ \\
\hline $4 d$ & $\begin{array}{l}\text { Proposed space station } \\
\text { receiving frequency }{ }^{6} \text { plan. }\end{array}$ \\
\hline
\end{tabular}

${ }^{2}$ Show all frequencies numerically in $\mathrm{Hz}, \mathrm{kHz}, \mathrm{MHz}$, or $\mathrm{GHz}$.

3 ITU emission designators are explained at: http://life.itu.int/radioclub/rr/ap01.htm. (Thank you, 4U1ITU.) Effect of Doppler shift is NOT included when determining bandwidth.

4 If using a frequency changing transponder, indicate the transmitting bandwidth. Effect of Doppler shift is NOT included when determining bandwidth.

${ }^{5}$ Common emission description means terms like transponder, NBFM, PSK31, 1200 baud packet (AFSK on FM), etc.

${ }^{6}$ Show all frequencies numerically in $\mathrm{Hz}, \mathrm{kHz}, \mathrm{MHz}$, or $\mathrm{GHz}$. 


\begin{tabular}{|c|c|c|}
\hline & $\begin{array}{l}\text { List for each frequency or } \\
\text { frequency band: } \\
\rightarrow \text { frequency or frequency band } \\
\rightarrow \text { ITU emission designator } \\
\rightarrow \text { common description of the } \\
\text { emission } \\
\rightarrow \text { noise temperature } \\
\rightarrow \text { associated antenna gain and } \\
\text { pattern }\end{array}$ & \\
\hline $4 \mathrm{e}$ & $\begin{array}{l}\text { Physical structure. } \\
\text { General description, including } \\
\text { dimensions, mass, antennas and } \\
\text { antenna placement, whether } \\
\text { stabilized or tumbling, etc. Give } \\
\text { URL's for drawings. }\end{array}$ & \\
\hline $4 \mathrm{f}$ & $\begin{array}{l}\text { Functional Description. } \\
\text { Describe each sections function } \\
\text { within the satellite. }\end{array}$ & \\
\hline $4 g$ & $\begin{array}{l}\text { Power budget. } \\
\text { Describe each power source, } \\
\text { power consuming section, power } \\
\text { storage, and overall power budget. }\end{array}$ & \\
\hline 5 & TELECOMMAND (NOT published) & \\
\hline $5 a$ & $\begin{array}{l}\text { Telecommand frequency plan. } \\
\text { List: } \\
\rightarrow \text { space station telecommand } \\
\text { frequencies or frequency bands, } \\
\rightarrow \text { ITU emission designator(s) } \\
\rightarrow \text { common description of the } \\
\text { emission } \\
\rightarrow \text { link power budget(s) } \\
\overrightarrow{\text { cipher system }}\end{array}$ & \\
\hline $5 b$ & $\begin{array}{l}\text { Positive space station transmitter } \\
\text { control. } \\
\text { Explain how telecommand stations } \\
\text { will turn off the space station } \\
\text { transmitter(s) immediately, even in } \\
\text { the presence of user traffic and/or } \\
\text { space station computer system } \\
\text { failure. }\end{array}$ & \\
\hline
\end{tabular}




\begin{tabular}{|c|c|c|}
\hline & $\begin{array}{l}\text { NOTE: Transmitter turn off control } \\
\text { from the ground is absolutely } \\
\text { required. Good engineering } \\
\text { practice is to make this capability } \\
\text { independent of all other systems. } \\
\text { Be sure to read the paper available } \\
\text { at: } \\
\text { http://www.iaru.org/satellite/Control } \\
\text { lingSatellites v27.pdf. }\end{array}$ & \\
\hline $5 c$ & $\begin{array}{l}\text { Telecommand stations. } \\
\text { List telecommand stations, } \\
\text { including contact details, for } \\
\text { sufficient Earth command stations } \\
\text { to be established before launch to } \\
\text { insure that any harmful } \\
\text { interference caused by emissions } \\
\text { from a station in the amateur- } \\
\text { satellite service can be terminated } \\
\text { immediately. See RR } 25.11 \text { and } \\
\text { RR } 22.1\end{array}$ & \\
\hline $5 d$ & $\begin{array}{l}\text { Optional: Give the complete space } \\
\text { station turn off procedure. } \\
\text { As a service, the IARU Satellite } \\
\text { Advisor will keep the space station } \\
\text { turn off procedure as a backup for } \\
\text { your operation. Only the space } \\
\text { station licensee may request the } \\
\text { information. If interference occurs } \\
\text { and the licensee cannot be } \\
\text { located, the licensee grants the } \\
\text { Satellite Advisor permission to use } \\
\text { the turn off procedure. Please } \\
\text { note that the Satellite Advisor will } \\
\text { use his best efforts, but cannot } \\
\text { guarantee success. The space } \\
\text { station licensee is still held } \\
\text { responsible for the space station } \\
\text { transmitter(s) by the licensing } \\
\text { administration. }\end{array}$ & \\
\hline 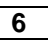 & (1) & \\
\hline $6 a$ & $\begin{array}{l}\text { Telemetry frequencies } \\
\text { List: } \\
\rightarrow \text { all telemetry frequencies or } \\
\text { frequency bands, } \\
\rightarrow \text { ITU emission designators } \\
\rightarrow \text { common description of the } \\
\text { emission } \\
\rightarrow \text { link budgets. }\end{array}$ & \\
\hline
\end{tabular}




\begin{tabular}{|l|l|l|}
\hline & $\begin{array}{l}\text { A URL with telemetry decoding } \\
\text { information. }\end{array}$ & \\
\hline $6 b$ & $\begin{array}{l}\text { Telemetry formats and equations. } \\
\text { Describe telemetry format(s), } \\
\text { including telemetry equations. } \\
\text { NOTE: Final equations must be } \\
\text { published as soon as available. }\end{array}$ & \\
\hline $6 c$ & $\begin{array}{l}\text { Is the telemetry transmission } \\
\text { format commonly used by radio } \\
\text { amateurs? If not, describe how } \\
\text { and where it will be published. }\end{array}$ & \\
& $\begin{array}{l}\text { Be sure to read: RR 25.2A. Text is } \\
\text { included in the paper available at: } \\
\text { http://www.iaru.org/satellite/sat- } \\
\text { freq-coord.html. }\end{array}$ & \\
\hline 7 & Launch plans (published) & \\
\hline $7 a$ & Launch agency & \\
\hline $7 b$ & Launch location & \\
\hline $7 c$ & $\begin{array}{l}\text { Planned orbit. } \\
\text { Include planned orbit apogee, } \\
\text { perigee, inclination, and period. }\end{array}$ & \\
\hline 7d & $\begin{array}{l}\text { List other amateur satellites } \\
\text { expected to share the same } \\
\text { launch. }\end{array}$ & \\
\hline
\end{tabular}

\section{Earth station information:}

\begin{tabular}{|l|l|l|}
\hline 8 & \multicolumn{2}{|l|}{ Typical Earth station - transmitting } \\
\hline $8 \mathrm{a}$ & $\begin{array}{l}\text { Describe a typical Earth station } \\
\text { used to transmit signals to the } \\
\text { planned space station. }\end{array}$ & $\begin{array}{l}\text { Show complete link budgets for all } \\
\text { Earth station transmitting } \\
\text { frequencies, except telecommand. }\end{array}$ \\
\hline 8b & \begin{tabular}{l} 
Link power budget. \\
\hline $\mathbf{9}$
\end{tabular} & $\begin{array}{l}\text { Typical Earth station - } \\
\text { receiving }\end{array}$ \\
\hline $9 a$ & $\begin{array}{l}\text { Describe a typical Earth station to } \\
\text { receive signals from the planned } \\
\text { satellite. }\end{array}$ \\
\hline $9 b$ & $\begin{array}{l}\text { Link power budget. } \\
\text { Show complete link budgets for all } \\
\text { Earth station receiving } \\
\text { frequencies. }\end{array}$ \\
\hline
\end{tabular}

\section{Additional information:}

Do not attach large files. Indicate the URL where the information is available. 
10 Please, supply any additional information that may assist the Satellite Advisor to coordinate your request(s).

\section{Certification:}

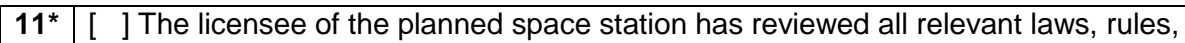
and regulations, and certifies that this request complies with all requirements to the best of his/her knowledge.

[ ] The licensee of the planned space station has reviewed all relevant laws, rules, and regulations and disagrees with IARU interpretations of Treaty requirements. The IARU Satellite Advisor is asked to consider the following interpretation. Explanation follows.

${ }^{*}$ Please tick appropriate box.

\section{Signature:}

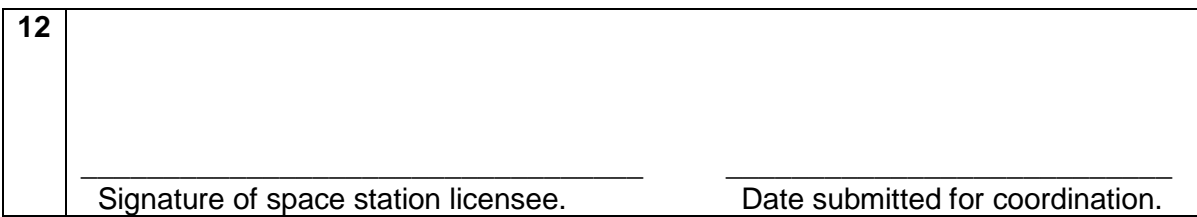




\title{
A.2 FCC Summary Template
}

\author{
[Satellite Name] Pre-Space Notification
}

Prepared for Joseph Hill, FCC

[Satellite Operator Name]

[University or Organization]

[Email]

[Phone Number]

[ Date] 


\section{A. PROCEDURES FOR ACQUIRING AMATEUR FREQUENCY LICENSE}

1 Spacecraft Overview

[Description of Satellite Mission]

[Mission Lifetime]

[Any pertinent payload information]

2 Electric Power System (EPS)

[Battery Protection Information]

[Batteries]

[Power Generation Information]

\section{Communications}

[Overview of Communication System]

[Modulation Scheme]

[Frequency and Coordinating Agency] 
[Data Rate]

[Output Power]

[Verification of Transmitter turn off capabilities]

[Location of Antenna]

\section{Command \& Data Handling (C\&DH)}

[Overview of Command and Data Handling]

[Main Processors]

[Sensors downlinked]

[Satellite inhibits]

[Satellite Interconnect protocols]

\section{Attitude Determination \& Control}

[Overview of $\mathrm{ADC}]$ 


\section{A. PROCEDURES FOR ACQUIRING AMATEUR FREQUENCY LICENSE}

6 Launch and Orbit

[Overview of Launch] Include as much as you have at time of filing.

[Launch location]

[Date of Launch]

\section{Volume and Mass}

[Overview of Mass and Volume Constraints]

[Structure Material]

[Side Panel Material]

[Size of Satellite]

[Mass]

[Any extensions or deployables]

8 Orbital Debris Mitigation 
[Output of NASA Debris Assessment software for mission life]

[Verification that orbital debris requirements were met]

\section{Debris Management}

[Verification that the Satellite will not produce orbital Debris]

\section{Accidental Explosion}

[Verification that the Satellite cannot explode]

\section{Source of Debris by Collision}

[Verification that the Satellite will not collide with another space object]

12 Casualty Risk from Reentry Debris Assessment

[Output of NASA Debris Assessment software for Casualty risk]

\section{Post-Mission Disposal}


A. PROCEDURES FOR ACQUIRING AMATEUR FREQUENCY LICENSE

[Plan for mission disposal after the satellite mission is over] 
Appendix B

Cubesat Survey Matrix 


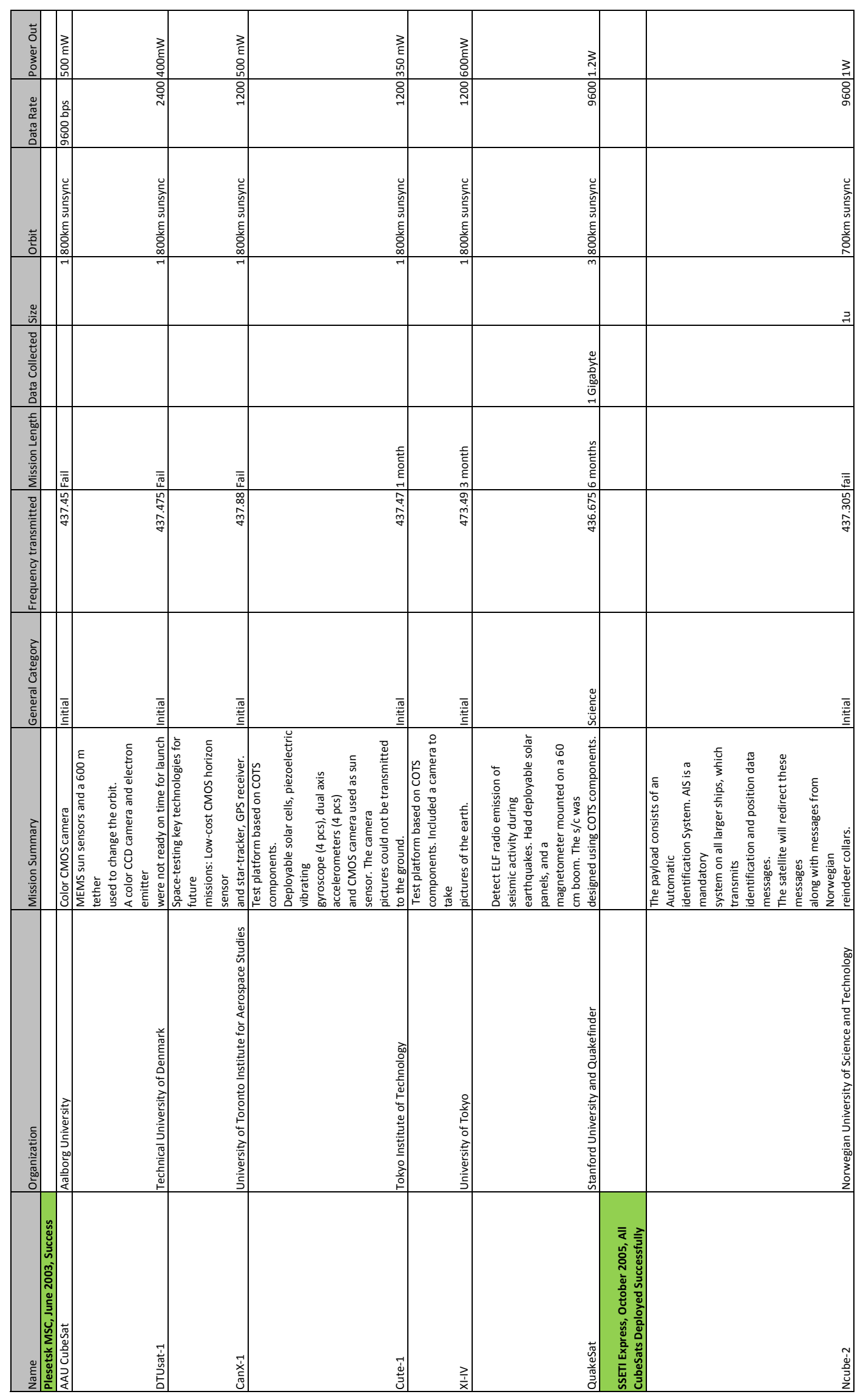




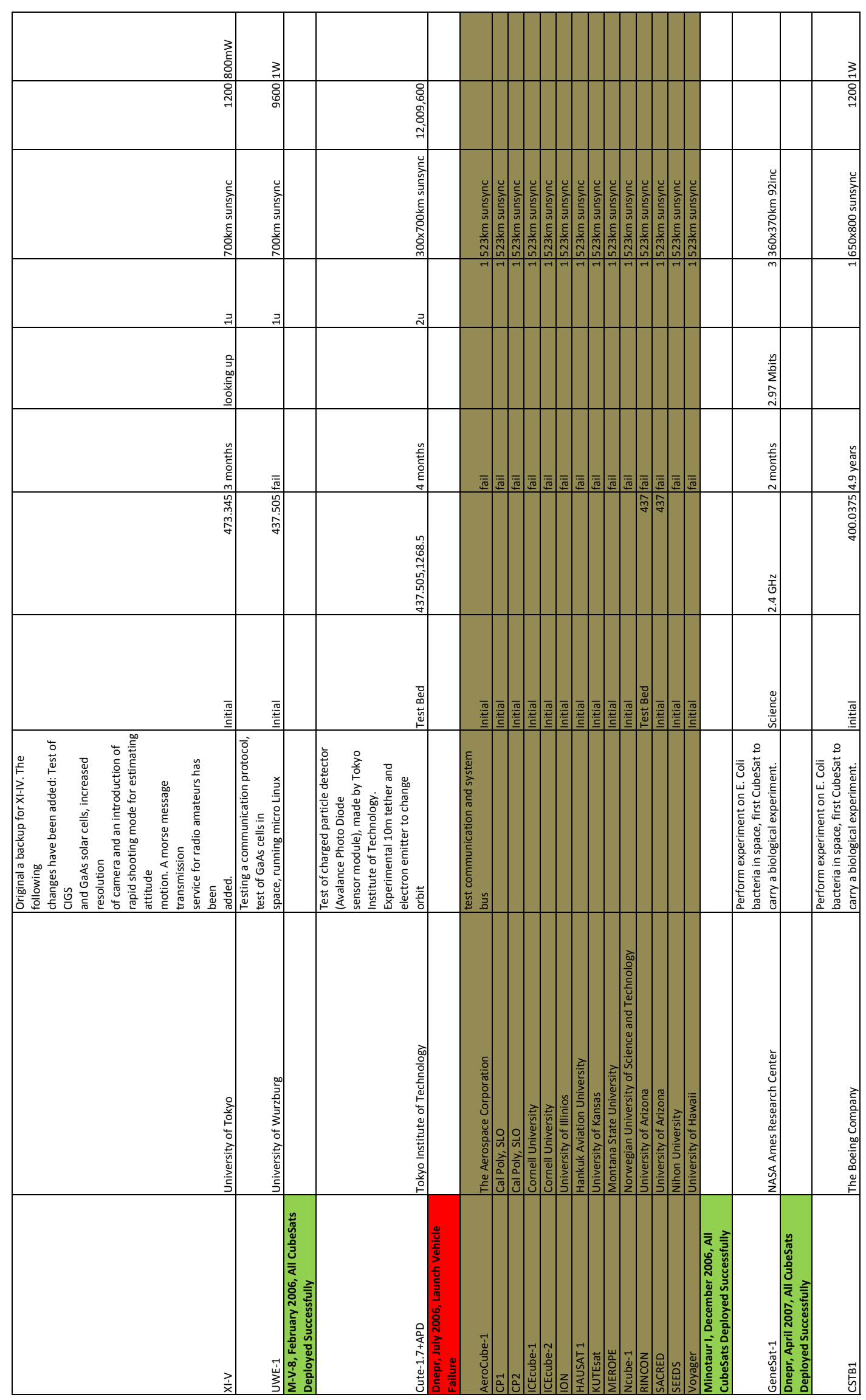




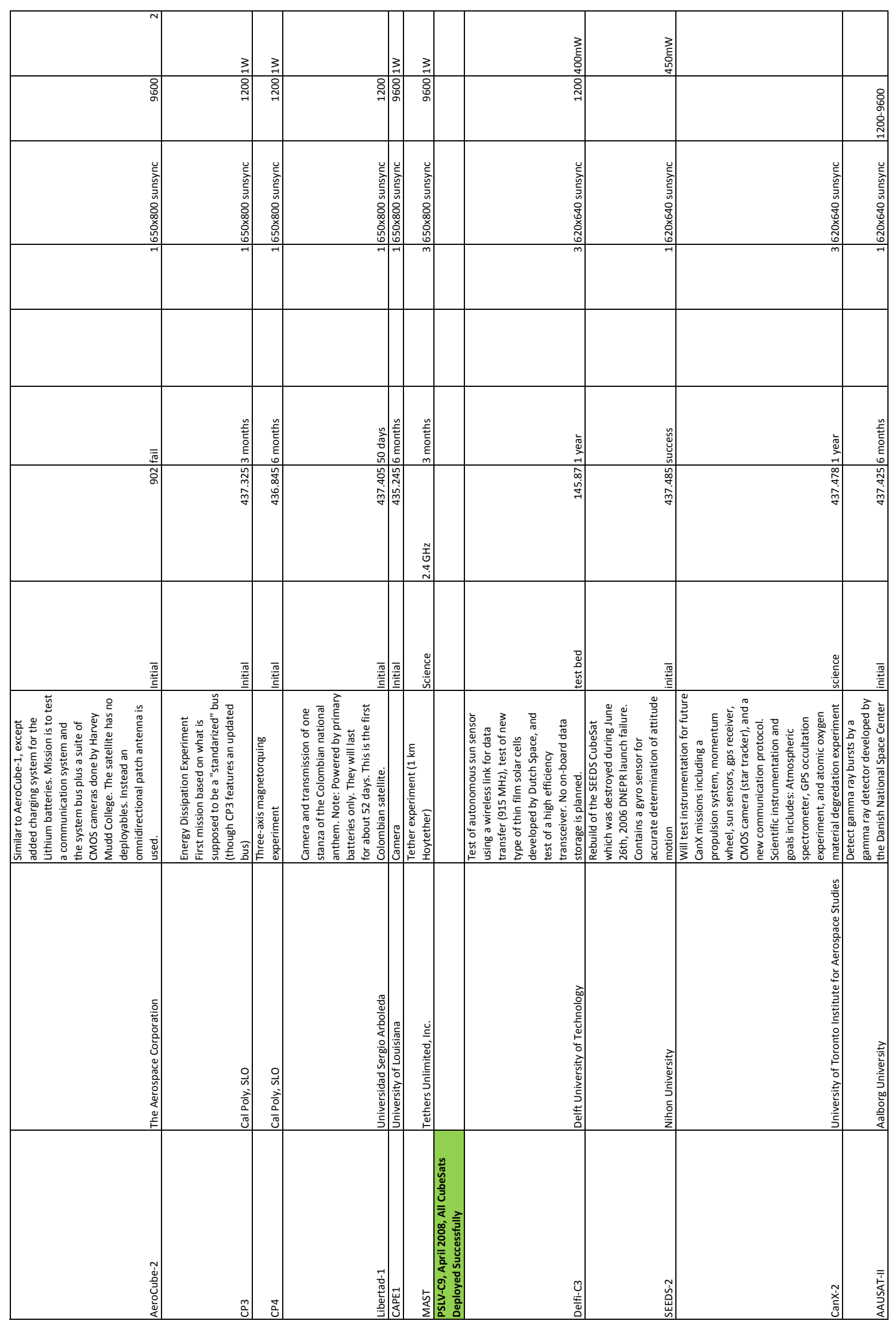




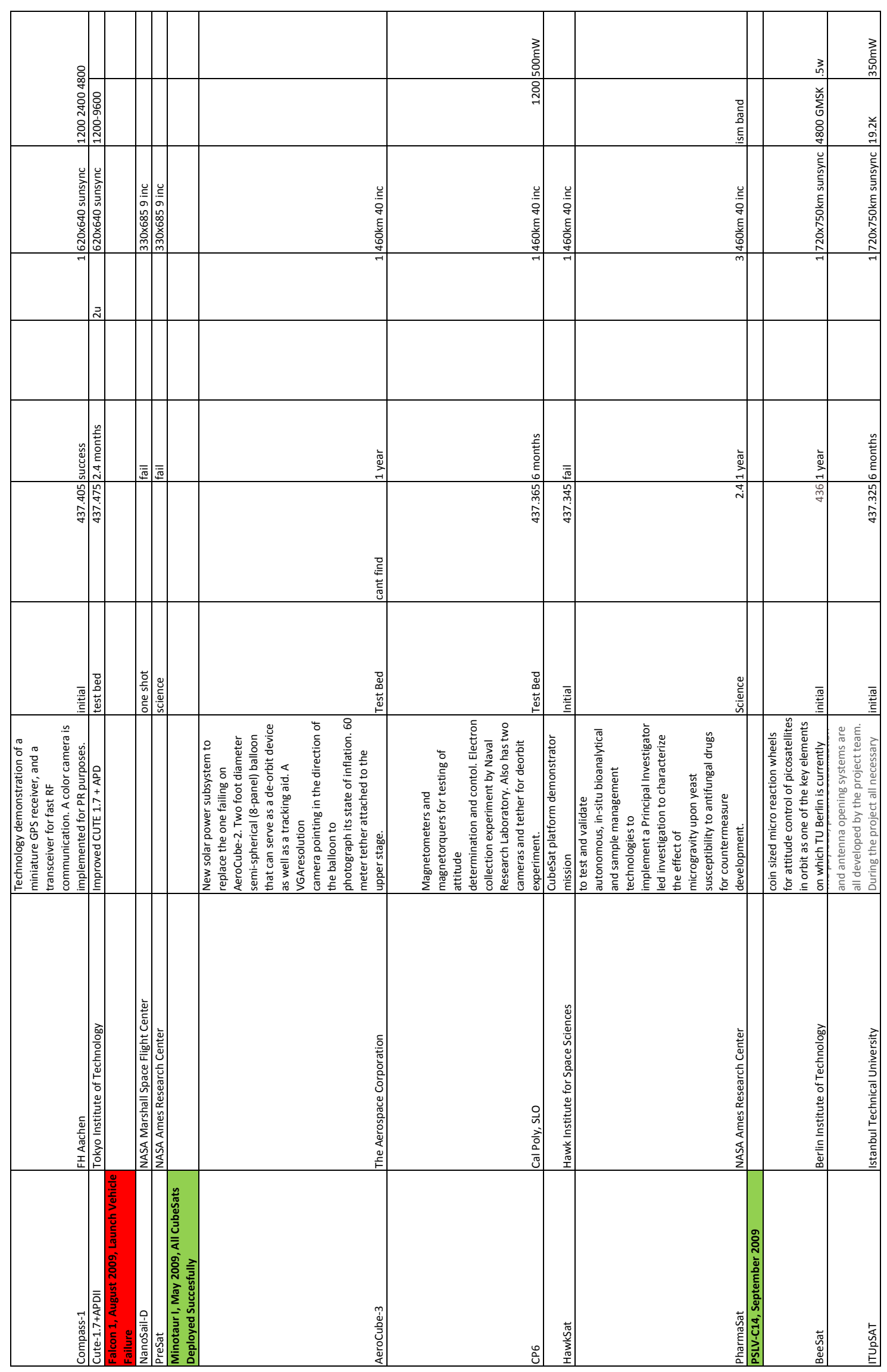




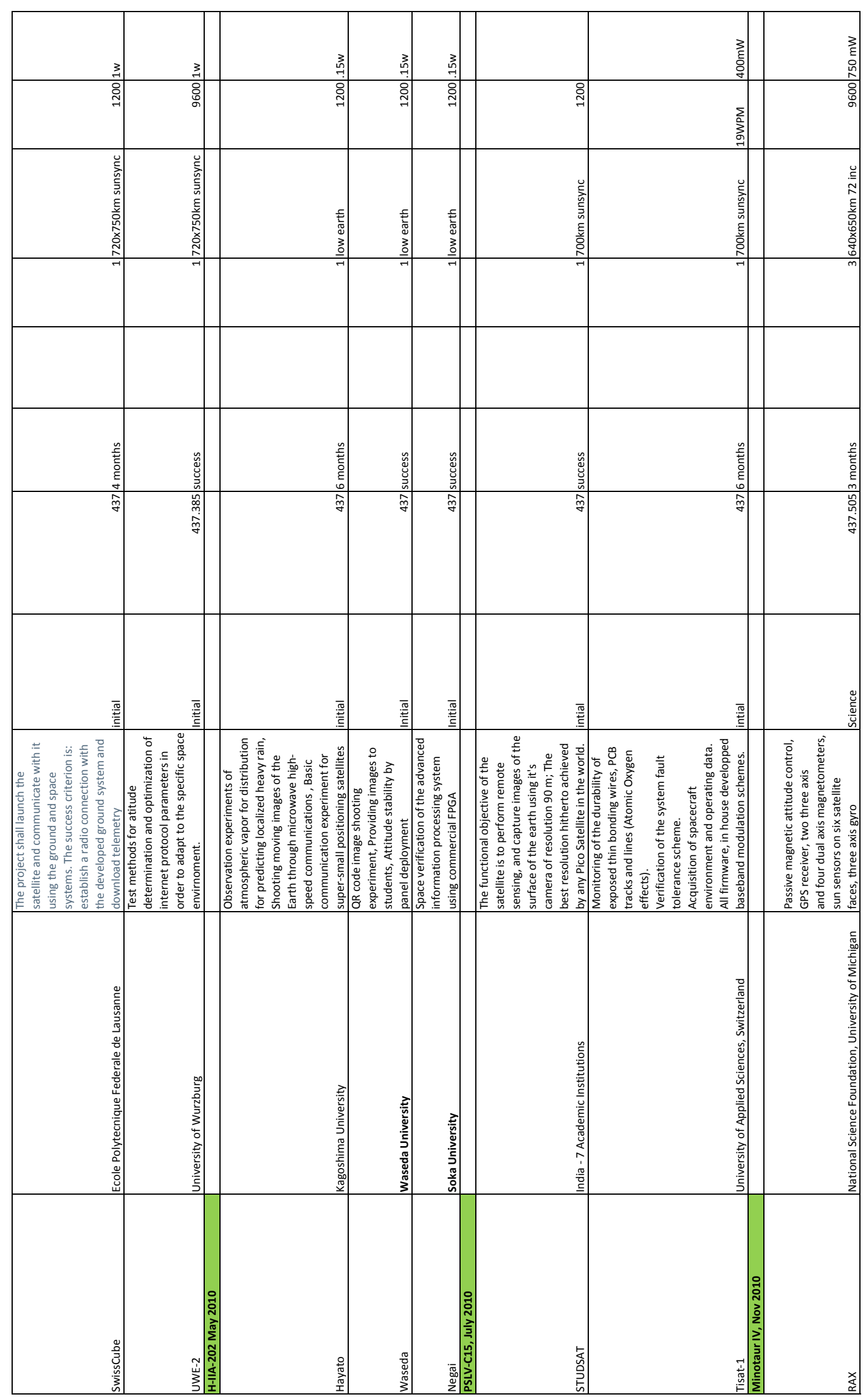




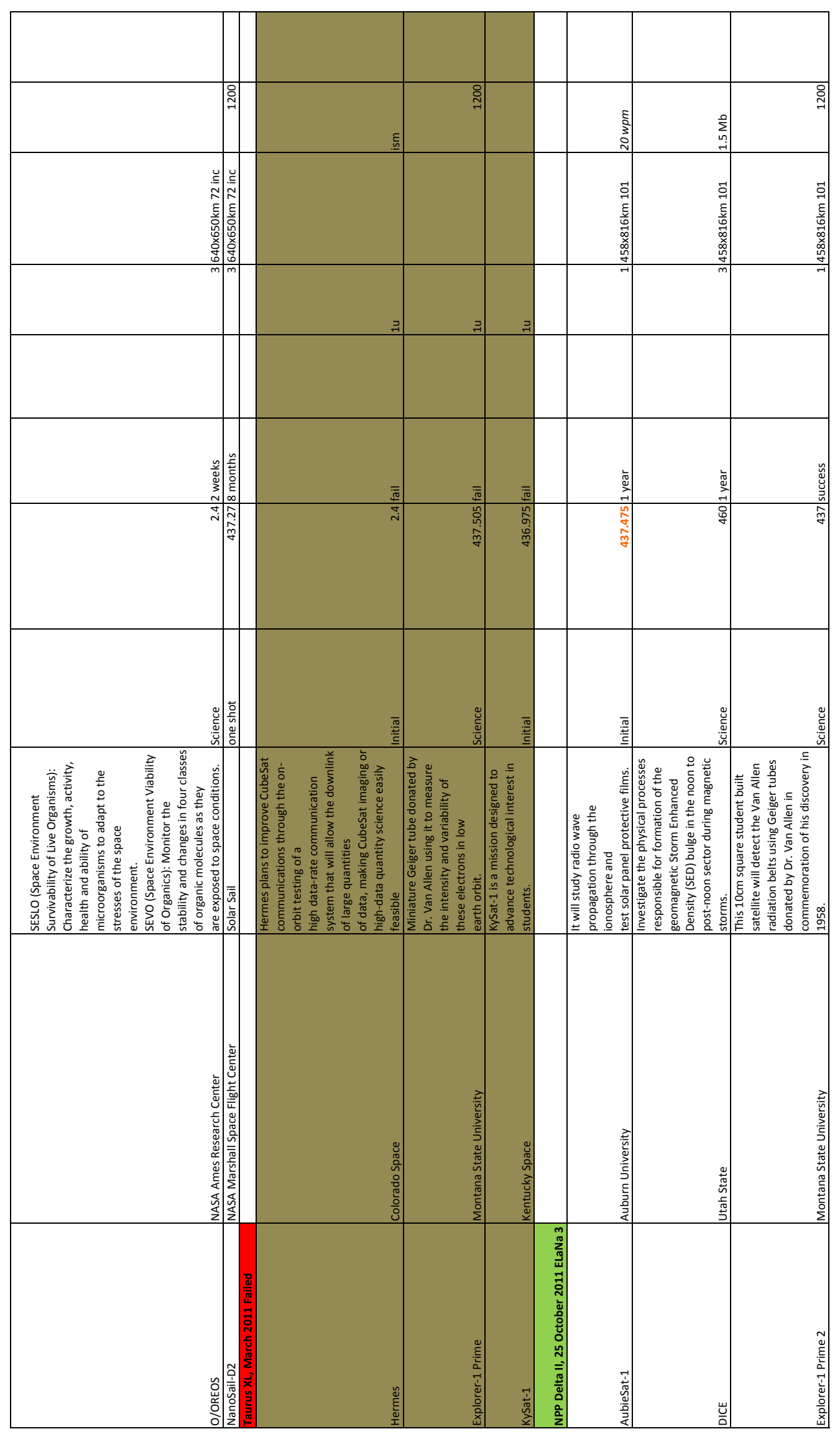




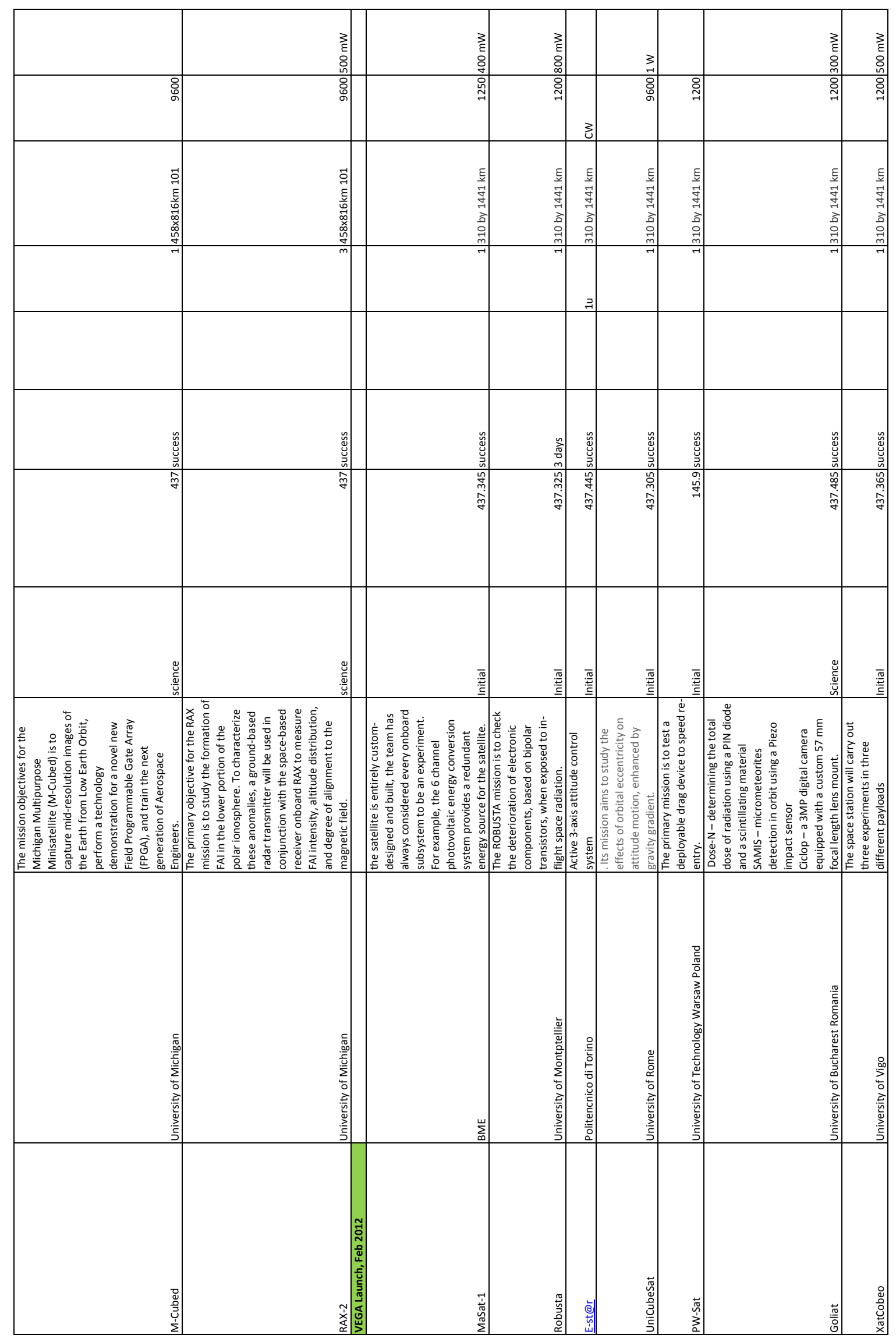


Appendix C

CMPT User Guide 


\section{Quick CMPT User Manual}

This document contains the steps required to setup and operate the CubeSat Mission Planning Toolbox (CMPT).

\section{Installation}

Here is a list of files and programs that need to be installed.

\section{Matlab files}

All Matlab files should be included in "CMPT_Matlab" and should be install where you normally run your Matlab files.

- access_time_needed.m

- acquire_initial_gnd_stn.m

- calc_packet_size_and_power_density.m

- cprintf.m

- disp_final_values.m

- ghostscript.m

- gnd_station.m

- gnd_station.fig

- gnd_station_max.m

- julian2greg.m

- month2num.m

- mylsfield.m

- num2month.m

- powerlinkb.m

- Print_menu.m

- Print_results.m

- satellite_new.m

- satellite_new.fig

- summary_dialog.m

- summary_dialog.fig

\section{STK files}

The STK files are all in the "STK_scenario" folder. At the time of creation, the folder needs to be placed directly in the C:\. For this code to run, the professional version of STK must be installed on your computer. Please contact AGI at support@agi.com to get information on licensing. 


\section{Setup}

This section discusses the steps needed to get the program running.

The first file you need to run is "satellite_new.m" this will open the window in figure 1. This window will allow you to pick a mission type, ground station option, orbit preference as well as other parameters. Changing the Mission type will change the options available to you. This is to limit the amount of over engineering. However, as your mission becomes more specific it will be helpful to select the custom option and adjust parameters to meet your needs.

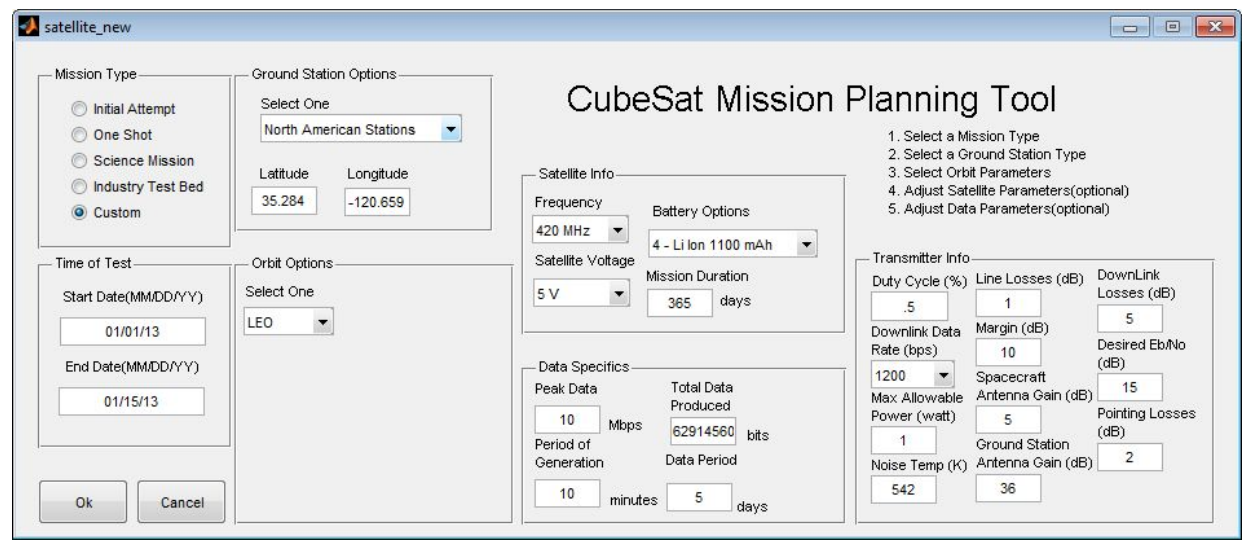

Figure 1. The Setup Window for CMPT

In the "Ground Station Option" panel, the latitude and longitude inputs allow you adjust the placement of your main ground station.

When using the custom mission type, changing the "peak data" or "period of generation" will adjust the total data produced. 


\section{Summary}

The Summary Window starts the simulation and provides a summary of options presented during the simulation. The graphs on the right do not have labels because of some issues with the Matlab GUI interface tool.

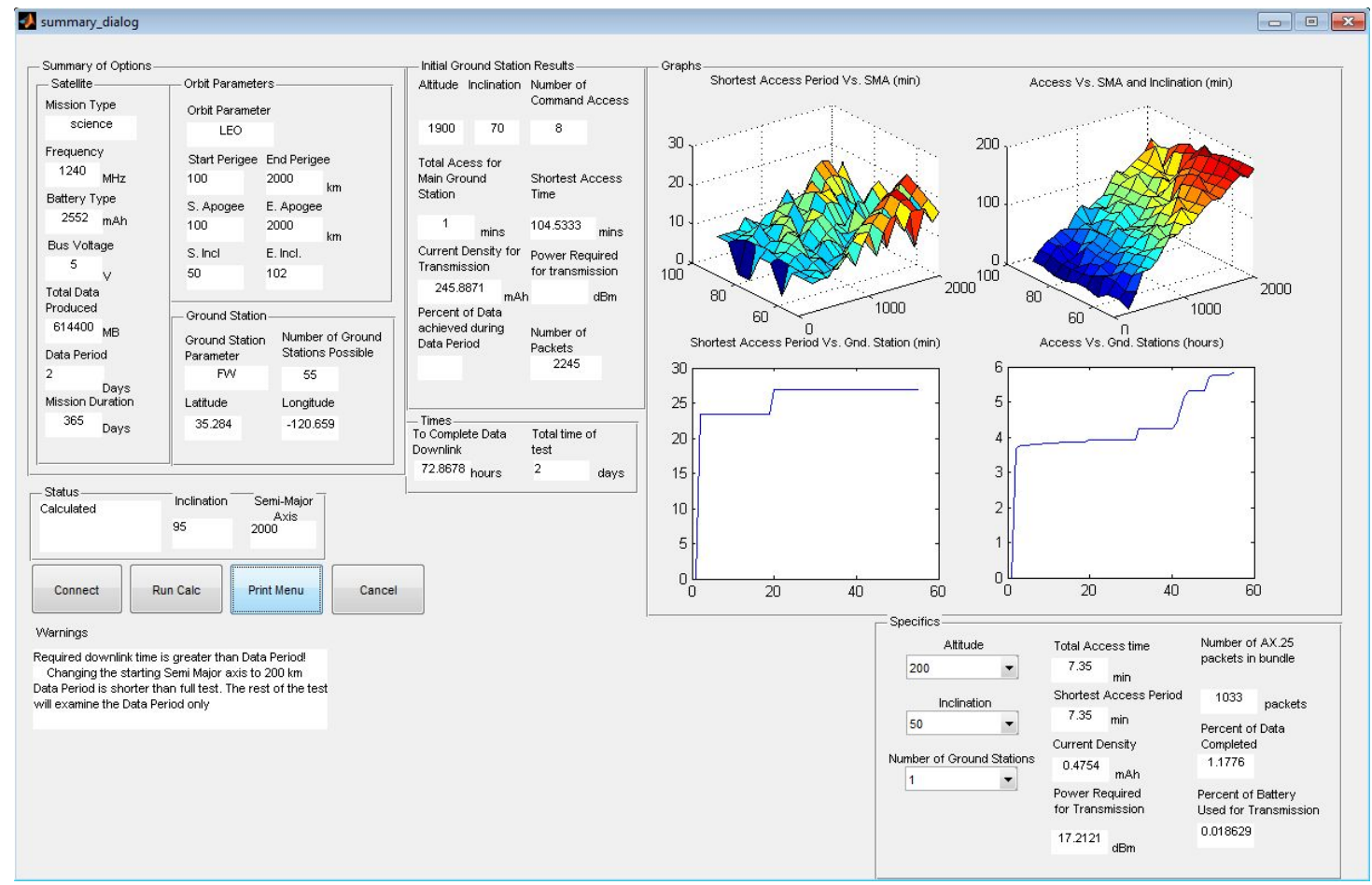

Figure 2. Summary Window

When the window opens click on the "Connect" to initiate connection with the STK and Matlab. Once the "Run Calc" becomes available click the button. This will start the simulation and it will take some time to run. Once the simulation has completed the "Print Menu" button will enable and allow for access to the last window. After the simulation has completed the "specifics" panel will open in the bottom right of the summary window. 


\section{Print Menu}

In the Print Menu Window, you are allowed to select and save graphs to jpg, see what values CMPT suggests you use for your mission planning and finally a text file with all the parameters in tabular form.

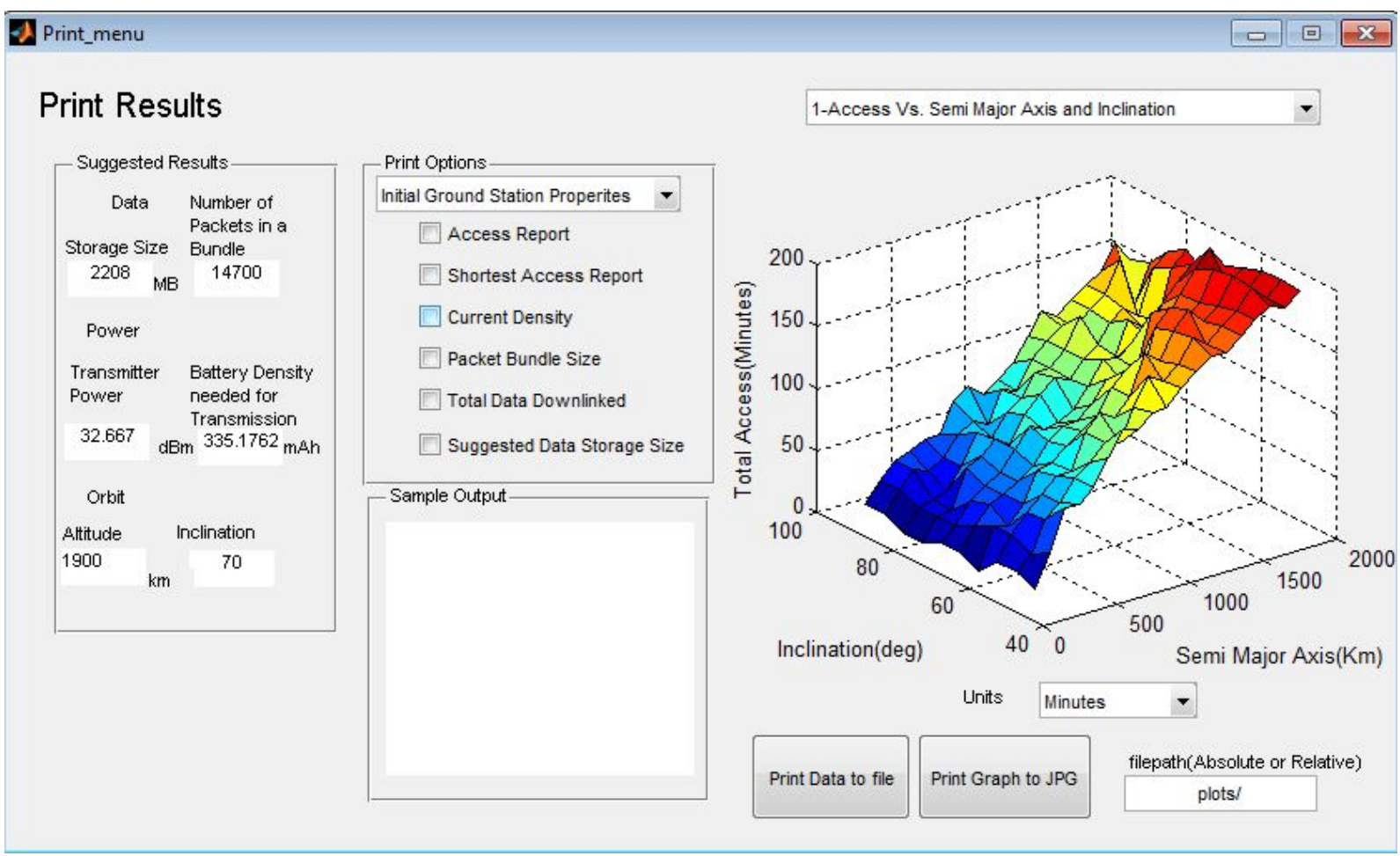

Figure 3. Print Menu Window

When printing graphs, you need to make sure the file path exists. CMPT will not create a folder for you. 
C. CMPT USER GUIDE 


\section{Appendix D}

\section{Useful MATLAB Code}

In this appendix, there are snippets of code that are useful to interfacing with STK. This includes initiating the simulation, getting the access report and converts the access report to a MATLAB friendly format. The access report gives the number of time periods in the report, and the start and stop time for each access period. The appendix follows the code presented in section 2.3.6. The code for sorting and processing the access reports is not included in this section. However, the code is available upon request. 


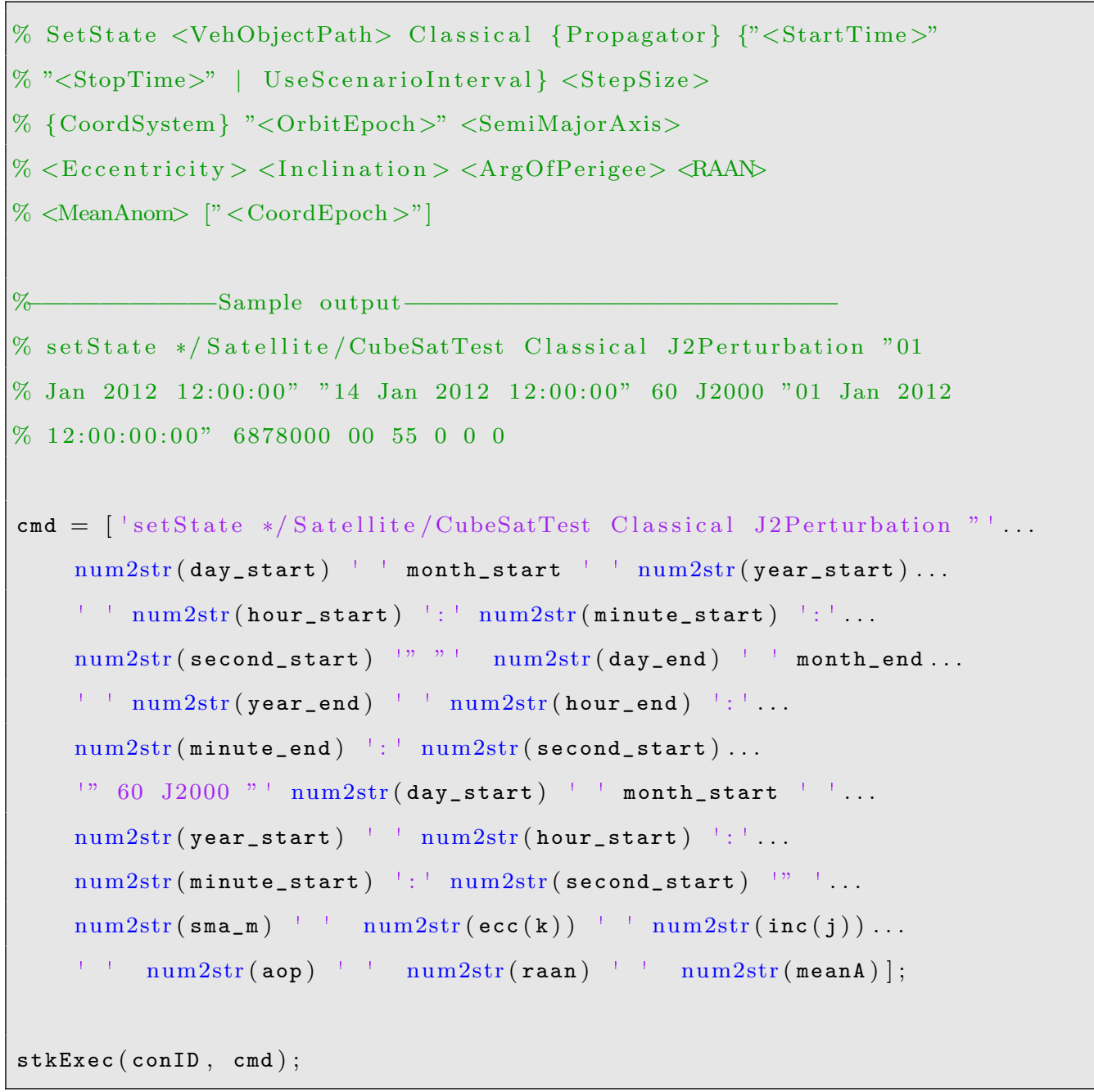

Figure D.1: MATLAB code used to run the simulation

In figure D.1 there is an example of how to initiate the simulation allowing a user to perform access calculations. The code above is required to run the code in the following figures. The first comment block shows the terms that are needed and the second comment block shows an example of the output from MATLAB. This output will connect and control STK if the code in section 2.3.6 was initilzed properly. 


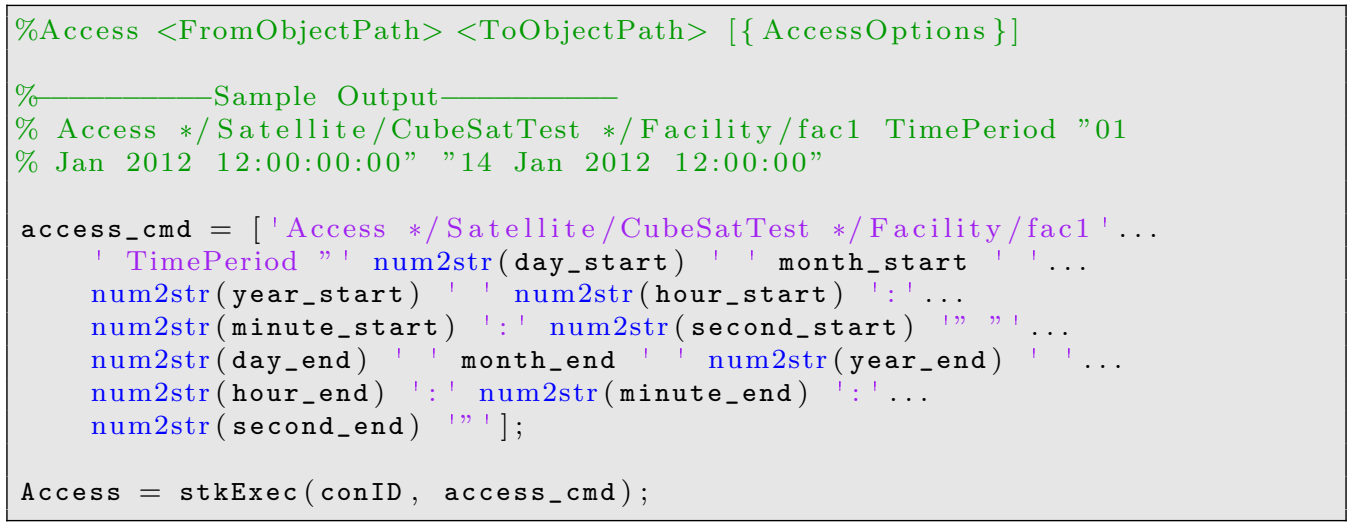

Figure D.2: MATLAB code used to get the Access Reports from STK.

The code in figure D.2 shows how CMPT gets the access reports from STK. Notice that the time period needs to be set in this command as well. It is possible to change the access period to examine different parts of the simulation. However, for CMPT and this thesis the time periods for the simulation and access reports are the same. 


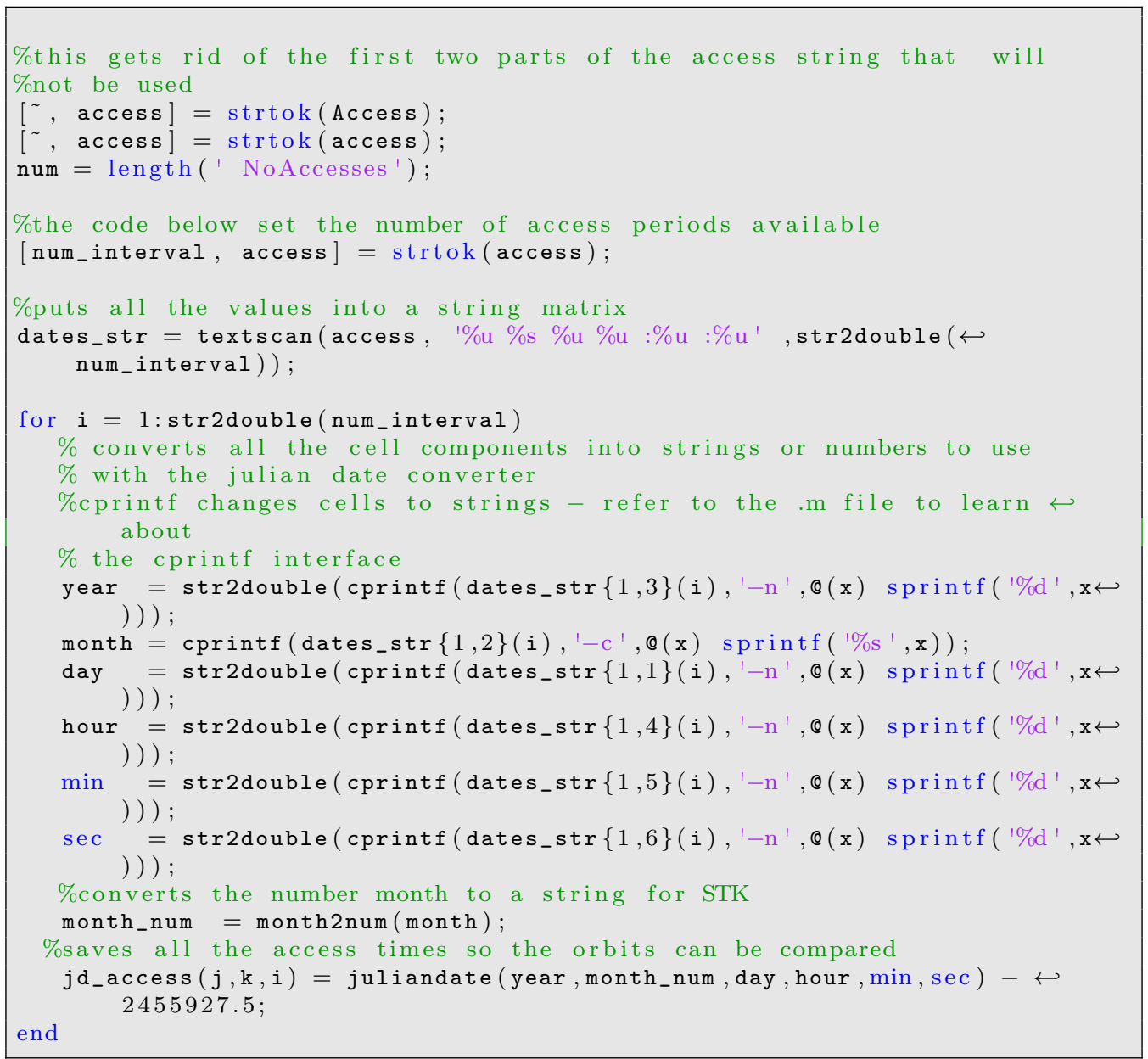

Figure D.3: MATLAB code used to convert the Access Reports from STK.

The code in figure D.3 shows how CMPT converts the output from STK into a format that is usable with MATLAB. All the access times are stored in $\mathrm{jd}$ _access $(\mathrm{j}, \mathrm{k}, \mathrm{i})$ where $\mathrm{j}$ is the index for the inclination, $\mathrm{k}$ is the index for the altitude and $\mathrm{i}$ is the increments through the matrix. 2455927.5 is the julian date of January 1, 2012, which is used to reduce the size of the julian number and make debugging much easier. Textscan is used to parse the report, however the output is saved in cells and cprintf is used to process the output of the textscan function. 


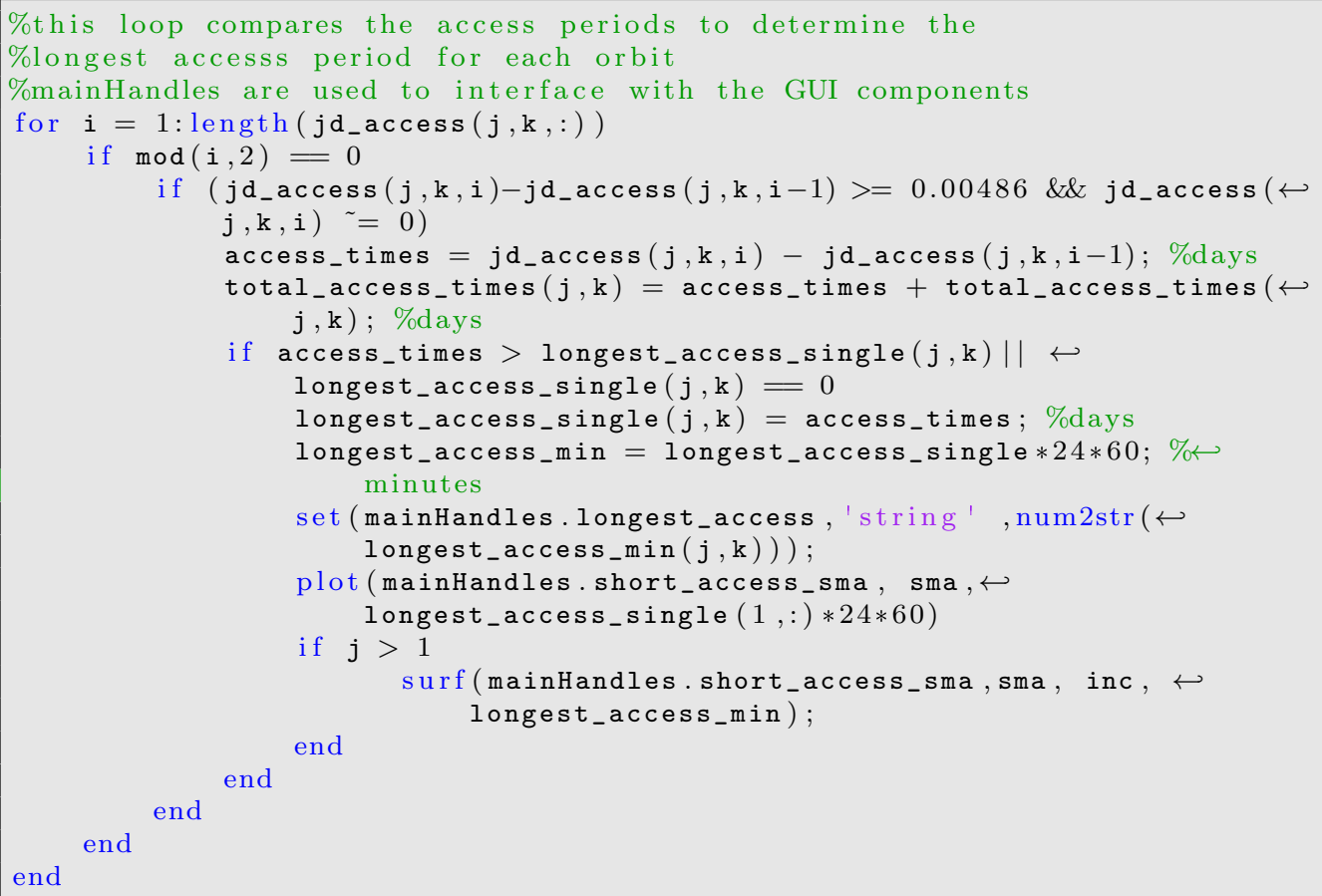

Figure D.4: MATLAB code used to find the longest access period in each orbit.

The code in figure D.4 shows how CMPT searches through each of the orbits to determine the longest access period. The longest access period is used to determine the best orbit. The longest access for each of the orbits is multiplied by the number of accesses and the highest total number is used as the best orbit option. 
Appendix E

CP6 Real World Data 


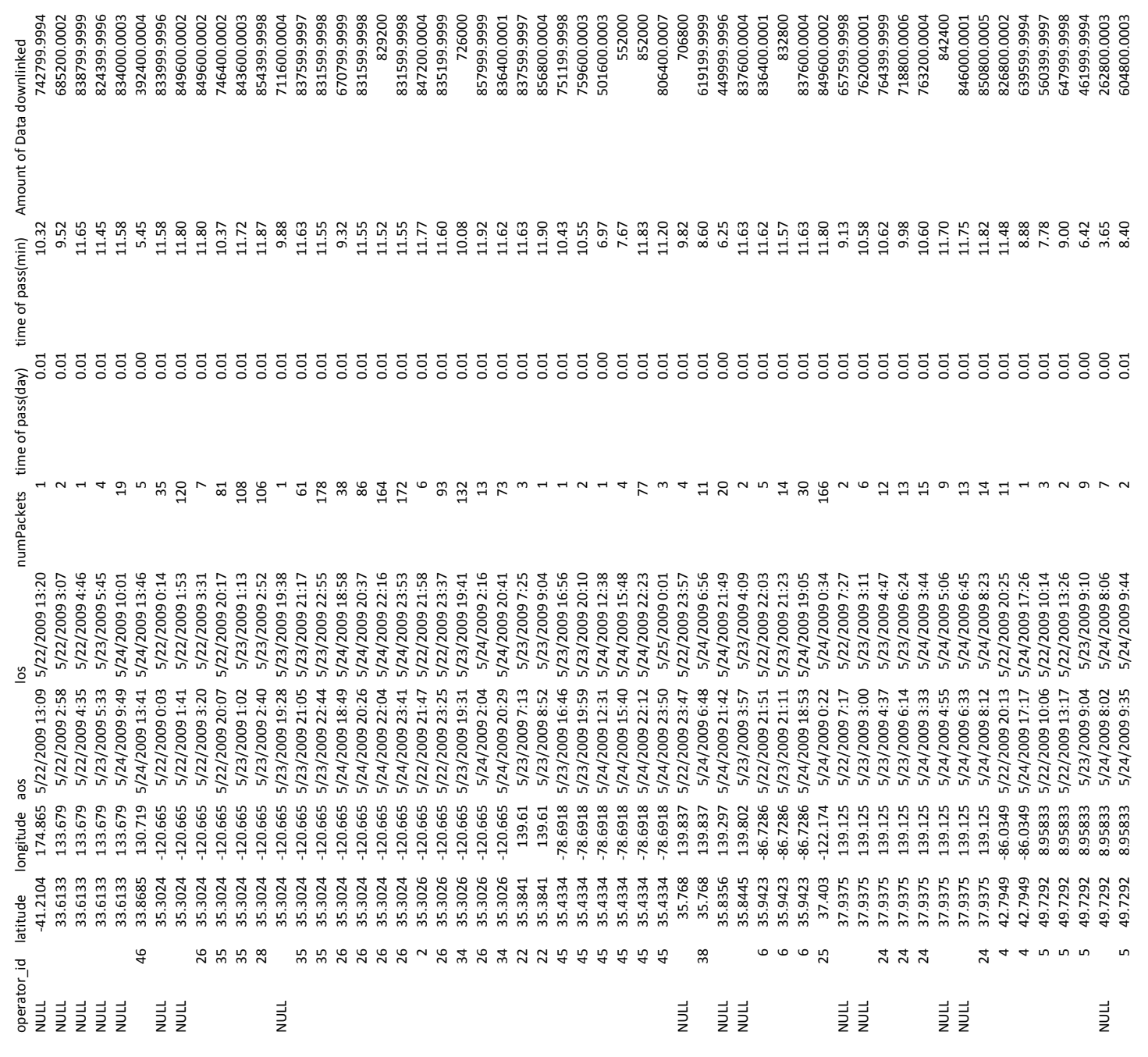




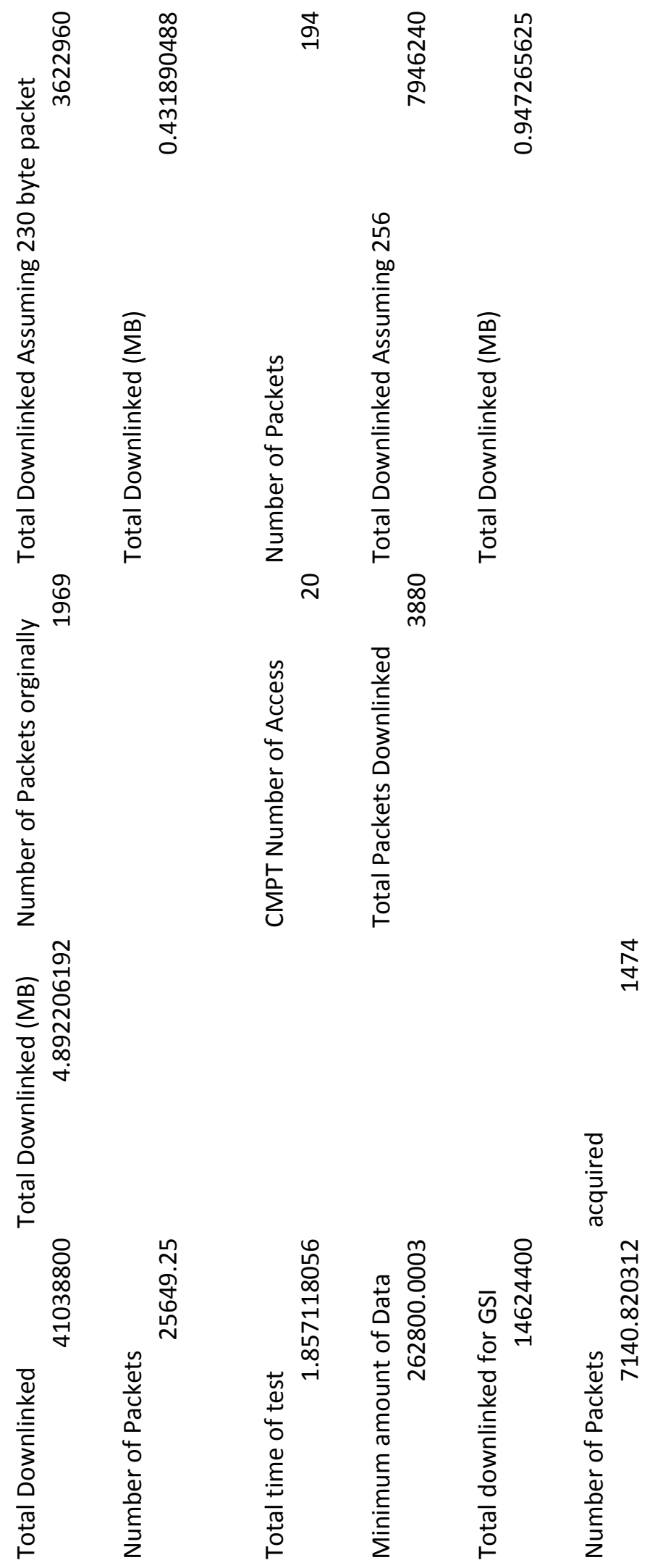

